\title{
CharaCterizing OPTICAL PROPERTIES IN FIBROUS TISSUES
}

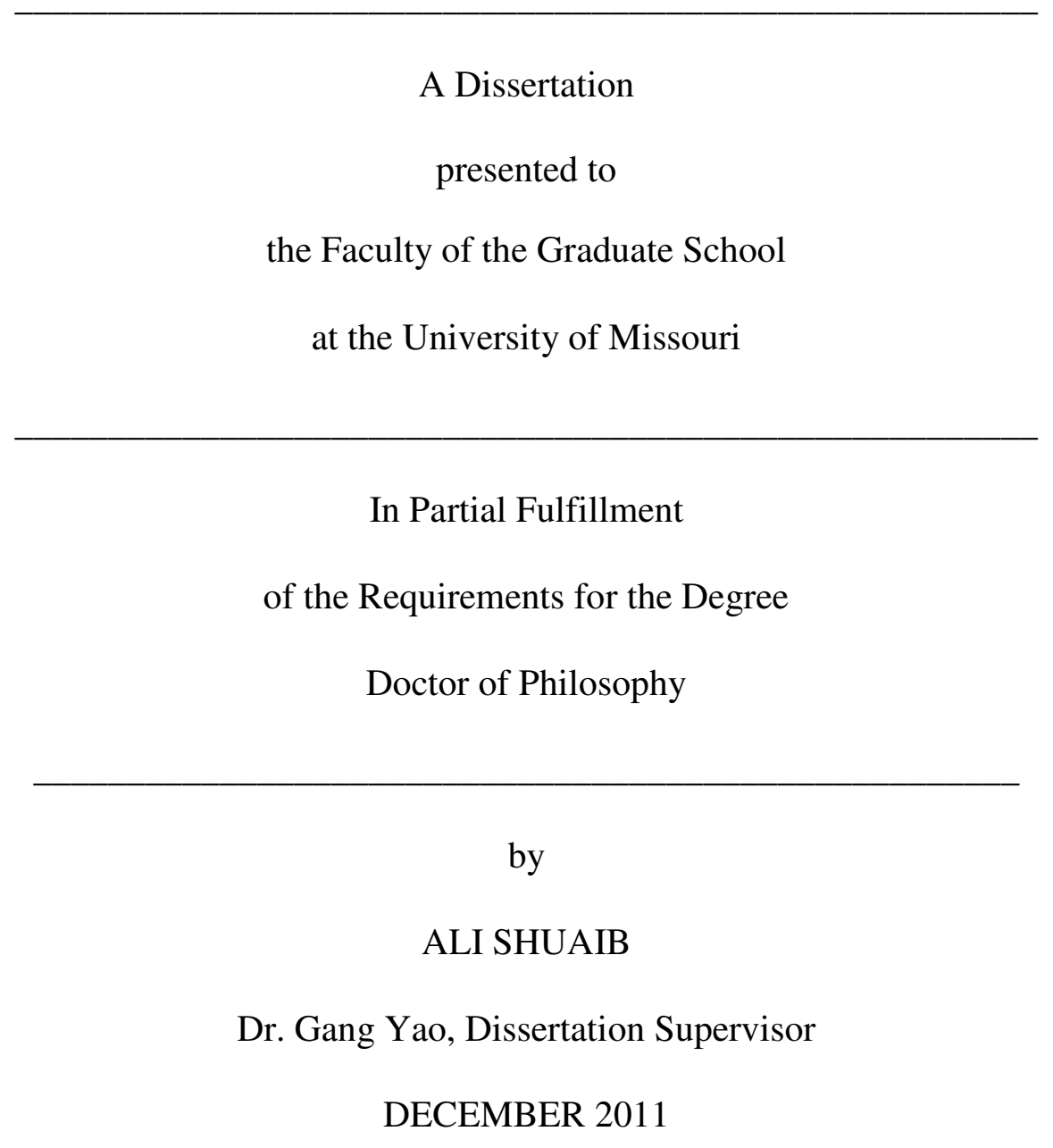


The undersigned, appointed by the dean of the Graduate School, have examined the dissertation entitled

\title{
CHARACTERIZING OPTICAL PROPERTIES IN FIBROUS TISSUES
}

\author{
Presented by Ali Shuaib, \\ A candidate for the degree of \\ Doctor of philosophy,
}

And hereby certify that, in their opinion, it is worthy of acceptance.

Associate Professor Gang Yao, Department of Biological Engineering

Professor Fu-hung Hsieh, Department of Biological Engineering

Professor Jinglu Tan, Department of Biological Engineering

Associate Professor Ye Duan, Department of Computer Science 


\section{ACKNOWLEDGMENTS}

The utmost gratitude goes to the members of my committee. This work would not have been possible without their support. I would especially like to thank Dr. Gang Yao for his encouragement and guidance during hard times and for believing in me even when I doubted myself. Also, I would also like to thank my other doctoral committee members, Drs. Jinglu Tan, and Fu-hung Hsieh, and Ye Duan, for their constant support and encouragement.

The help of my fellow labmates, Janaka C. Ranasinghesagara, Chuanmao Fan, Jinjun Xia, Chathuri Daluwatte, Xiaofei Fan, and Randima Dinalankara, was instrumental in broadening my horizons and making long days in the lab more bearable. I would like to thank meat lab manager Rick Disselhorst for his support to provide tissue samples. My appreciation also goes to all the Biological Engineering staff for the many services they so graciously performed throughout my stay at MU.

In addition, I would like to acknowledge the financial support from Kuwait University. Finally, I extend my greatest thanks to my family; they have been of the utmost support to me. 


\section{TABLE OF CONTENTS}

ACKNOWLEDGMENTS

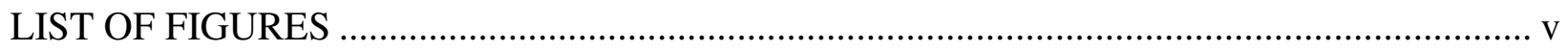

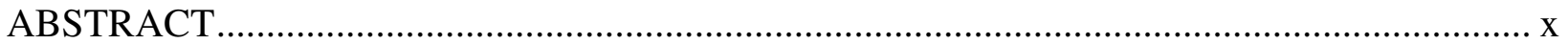

\section{CHAPTER}

1. INTRODUCTION AND BACKGROUND ......................................................... 1

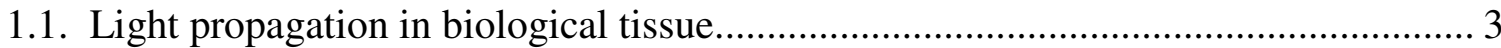

1.1.1. Isotropic diffuse theory ..................................................................... 3

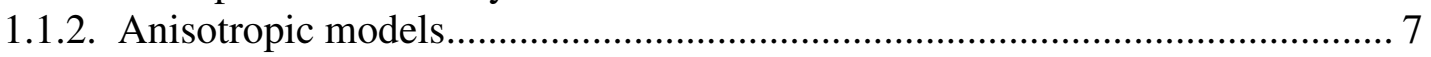

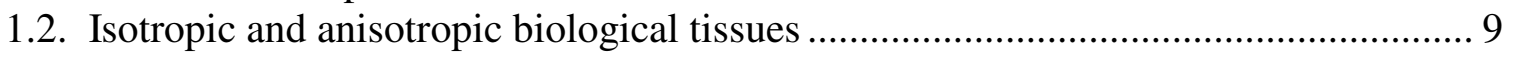

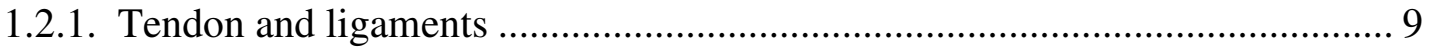

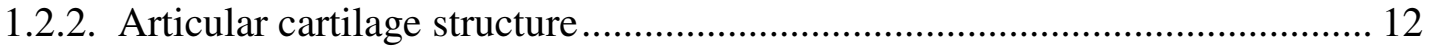

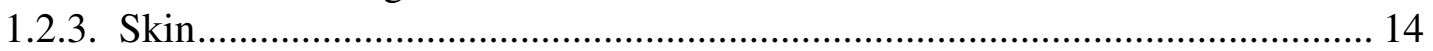

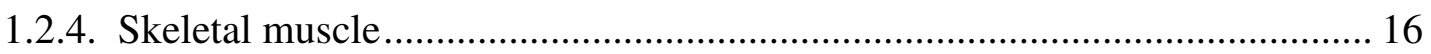

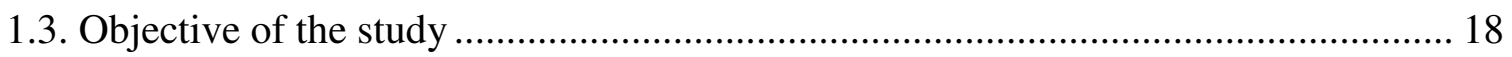

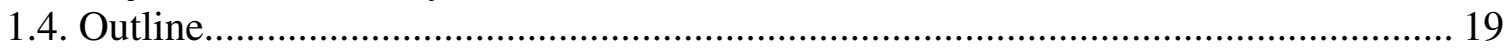

2. EQUI-INTENSITY DISTRUBTION OF OPTICAL REFLECTANCE IN FIBROUS

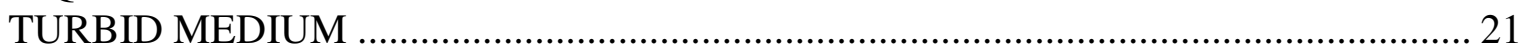

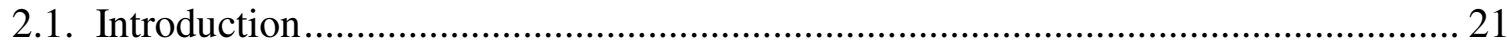

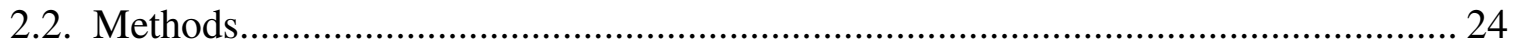

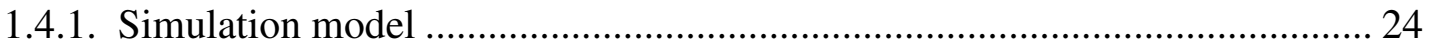

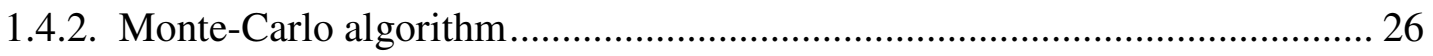

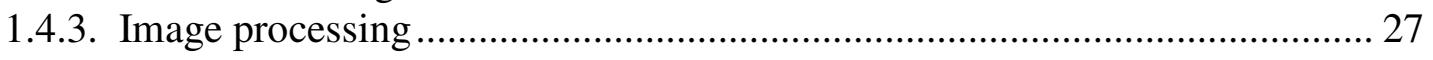

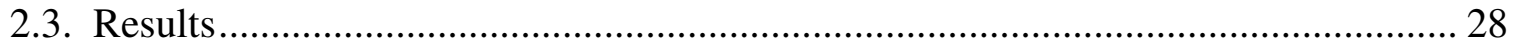

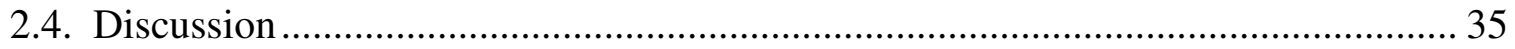

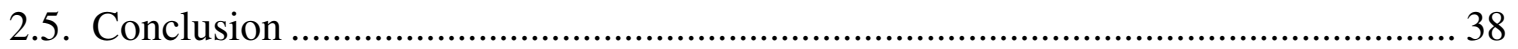

3. DETERMINING THE OPTICAL PROPERTIES IN A FIBROUS TURBID MEDIUM

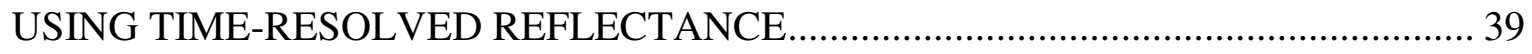

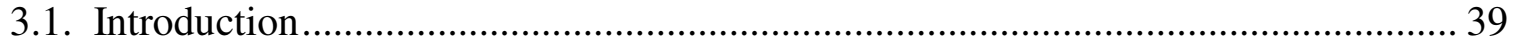

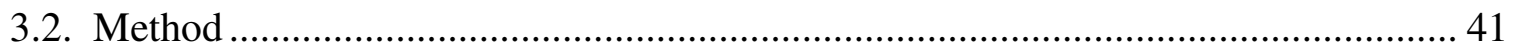

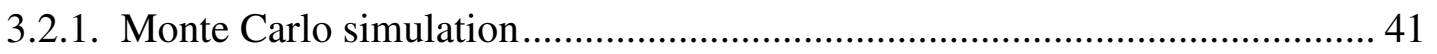

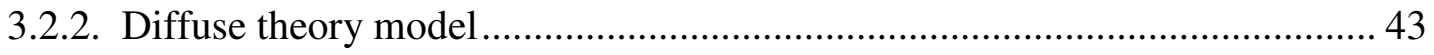

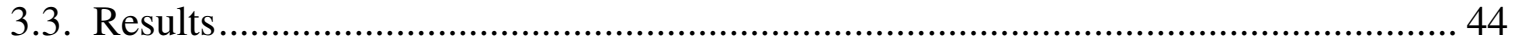

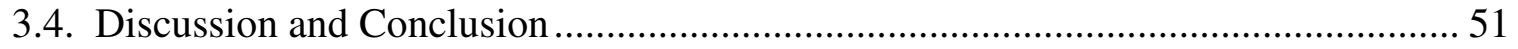


4. COMPARISON OF ISOTROPIC AND ANISTROPIC DIFFUSE MODELS IN DETERMINING OPTICAL PROPERTIES IN A FIBROUS MEDIUM USING TIME-

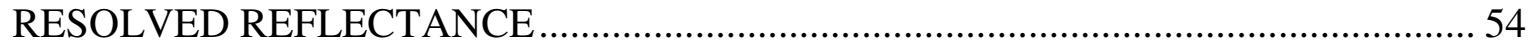

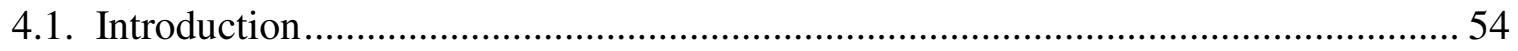

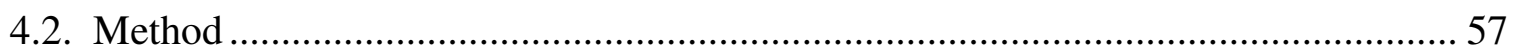

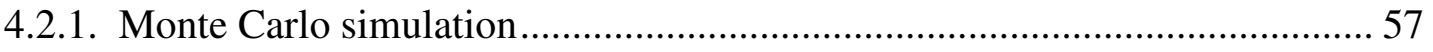

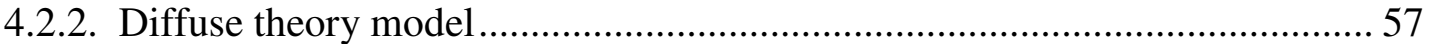

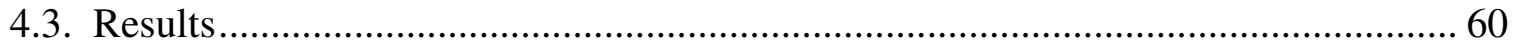

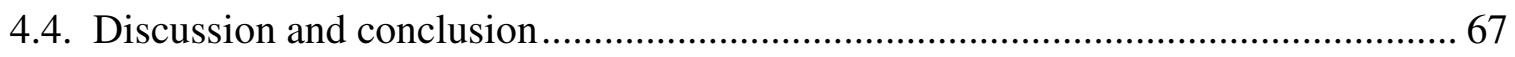

5. MEASURING PATH-LENGTH RESOLVED REFLECTANCE USING LOWCOHERENCE MACH-ZEHNDER INTERFEROMETRY ......................................... 70

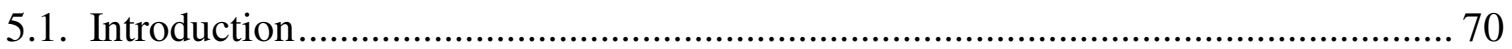

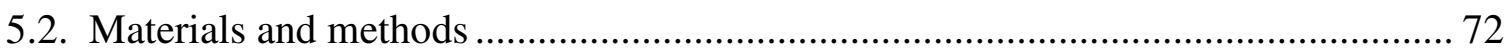

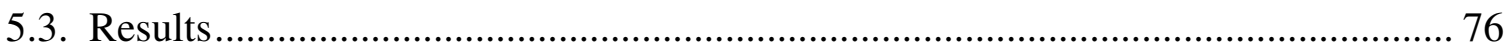

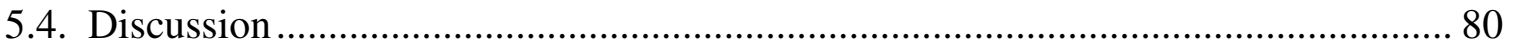

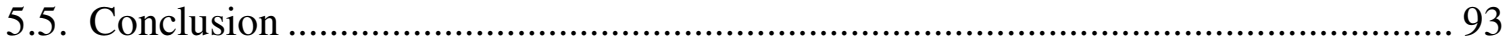

6. MEASURING TENDON OPTICAL PROPERTEIS BY USING PATH-LENGTH

RESOLVED REFLECTANCE .................................................................... 95

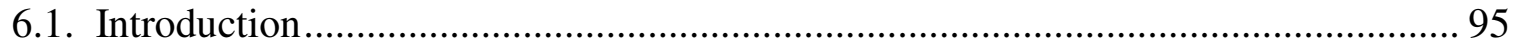

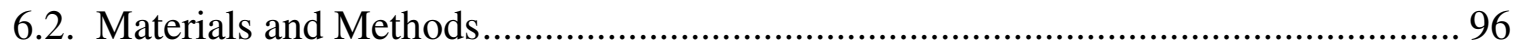

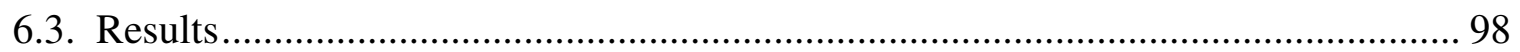

6.4. Discussion ............................................................................................... 105

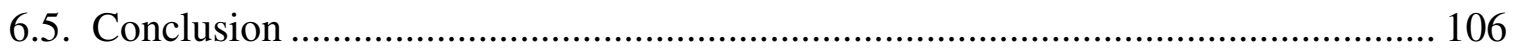

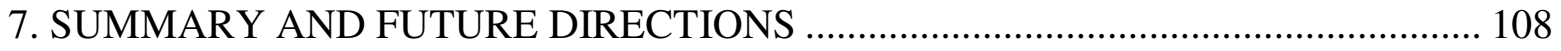

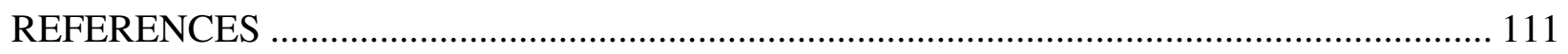

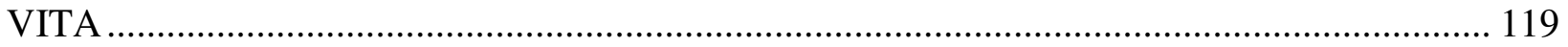




\section{LIST OF FIGURES}

Figure

Page

1-1: Geometry for the calculation of $R(\rho, \mathrm{t})$ for a semi-infinite isotropic medium. The incident beam is assumed to create an isotropic photon source at depth $z_{o}$, indicated by the close circle. The boundary condition can be met by adding a negative source indicated by the open circle....

1-2: (a) Hierarchical structure of tendon. A tendon consists primarily of collagen. The illustration is sketched from (Kastelic et al. 1978). Light microscopy of a tendon in (b) longitudinal section. Regularly arranged fibers " $f$ " are mixed with fibroblasts (arrow). Hematoxylin-Eosin (H\&E) stain magnified 40 times of its original size.

1-3: The illustration, showing collagen organization in the articular cartilage, is sketched from (Jazrawi et al. 2011).

1-4: The anatomy of normal skin is shown here. The epidermis lies above the dermis, which lies above the subcutaneous fat or "fat cells". This illustration is sketched from (Victoria 2010).

1-5: (a) The hierarchy of the skeletal muscle. (b) Illustration of sarcomere structure. These illustrations are sketched from (Lieber 2002).

2-1: (a) Illustration of the scattering medium, which is composed of infinite long cylinders and uniformly distributed spherical particles. (b) Schematic diagram of the simulated experimental setup.

2-2: Sample reflectance images obtained in (a) isotropic and (b) anisotropic mediums. The cylinders were aligned with the y axis (vertical direction). The dash lines were sample fitting results using Eq. (5). The fitting parameters $\beta$ and $\mathrm{q}$ were shown as a function of the distance along the $y$ axis in the above (c) isotropic and (d) anisotropic media.

2-3: The fitted axes ratio $\beta$ versus the ADE prediction of $\sqrt{\mu_{s}^{\prime}(x) / \mu_{s}^{\prime}(y)}$ in anisotropic media of three different cylinder radii: $0.1,0.25$, and $1.5 \mu \mathrm{m}$. The background optical properties used in the simulation were $\mu_{\mathrm{s}, \mathrm{b}}=30 \mathrm{~cm}^{-1}, \mathrm{~g}_{\mathrm{b}}=0.8$, and $\mu_{\mathrm{a}}$ $=0.01 \mathrm{~cm}^{-1}$. 
2-4: The fitted axes ratio $\beta$ versus cylinder radius. $\sqrt{\mu_{s}^{\prime}(x) / \mu_{s}^{\prime}(y)}$ was maintained at constant value of 1.08. The background optical properties used in the simulation were $\mu_{s, b}=30 \mathrm{~cm}^{-1}, \mathrm{~g}_{\mathrm{b}}=0.8$, and $\mu_{\mathrm{a}}=0.01 \mathrm{~cm}^{-1}$

2-5: The fitted axes ratio $\beta$ and total diffuse reflectance at different (a) background scattering coefficient $\mu_{\mathrm{s}, \mathrm{b}}$, (c) background anisotropy $g_{\mathrm{b}}$, and (e) absorption coefficient $\mu_{\mathrm{a}}$. The corresponding transition distances were shown in (b), (d), and (f), respectively. Unless otherwise indicated, the optical properties in the simulation were $r=0.1 \mu \mathrm{m} ; \mu_{\mathrm{s}, \mathrm{b}}=30 \mathrm{~cm}^{-1} ; g_{\mathrm{b}}=0.8 ; \mu_{\mathrm{a}}=0.01 \mathrm{~cm}^{-1}$ and $\sqrt{\mu_{s}^{\prime}(x) / \mu_{s}^{\prime}(y)}=1.08$.

2-6: (a) The scattering efficiency $Q_{s}$ and (b) anisotropy $g_{c}$ as a function of incident angle $\xi$ for cylinders of different radii.

3-1: The schematic diagram of the sample model used in the Monte Carlo

3-2: (a) Sample spatial resolved reflectance image obtained in anisotropic medium. The cylinders were aligned along the $y$-axis. The image size is $1 \times 1 \mathrm{~cm}^{2}$. (b) The time-resolved measurements parallel $\left(0^{\circ}\right)$ and perpendicular $\left(90^{\circ}\right)$ to the fiber direction (solid lines) at $\rho=0.95 \mathrm{~cm}$ and their isotropic diffuse model (circles).

3-3: The derived reduced scattering coefficients (a) parallel $\mu_{\mathrm{s}, 0}^{\prime}$ and (b) perpendicular $\mu_{\mathrm{s}, 90}^{\prime}$ to the fiber direction as a function of the total reduced scattering coefficient, $\mu_{s, t}^{\prime}$. (c) The derived absorption coefficients parallel $\mu_{a, 0}$ and perpendicular $\mu_{a, 90}$ to the fiber direction as a function of actual total reduced scattering coefficient, $\mu_{s, t}^{\prime}$. The optical properties used in the simulation were $\mu_{s, c}^{\prime}=2,3,5,8$ and $10 \mathrm{~cm}-1, \mu_{s, b}^{\prime}=8 \mathrm{~cm}^{-1}, g_{b}=0.8$, and $\mu_{a}=0.1 \mathrm{~cm}^{-1}$. The dotted lines indicate the theoretical values.

3-4: The derived reduced scattering coefficients parallel $\mu_{s, 0}^{\prime}$ and perpendicular $\mu_{s, 90}^{\prime}$ to the fiber direction at different (a) background scattering coefficients $\mu_{s, b}$, (c) background anisotropies $g_{b}$, and (e) absorption coefficients $\mu_{a}$. The corresponding average derived absorption coefficients are shown in (b), (d), and (f), respectively. Unless otherwise indicated, the optical properties in the simulation were $r=0.1 \mu \mathrm{m}, \mu^{\prime}{ }_{s, b}=8 \mathrm{~cm}^{-1}, g_{b}=0.8, \mu_{a}=0.1 \mathrm{~cm}^{-1}$, and $\sqrt{\mu_{s}^{\prime}(x) / \mu_{s}^{\prime}(y)}=1.275$.

3-5: The derived (a) reduced scattering coefficient parallel $\mu_{s, 0}^{\prime}$ and perpendicular $\mu_{s, 90}^{\prime}$ and (b) absorption coefficient parallel $\mu_{a, 0}$ and perpendicular $\mu_{a, 90}$ to the fiber direction at different cylinder refractive index. Unless otherwise indicated, the optical properties in the simulation were $r=0.1 \mu \mathrm{m}, \mu_{s, c}^{\prime}=13 \mathrm{~cm}^{-1} ; \mu_{s, b}^{\prime}$ $=8 \mathrm{~cm}^{-1}, g_{b}=0.8, \mu_{a}=0.1 \mathrm{~cm}^{-1}$, and $\sqrt{\mu_{s}^{\prime}(x) / \mu_{s}^{\prime}(y)}=1.275$. 50 
3-6: The corrected reduced scattering coefficient $\mu_{s, c}^{\prime}$ obtained by using the Eq. (3-3) compared with the true cylinder reduced scattering coefficient $\mu_{s, c}^{\prime}\left(90^{\circ}\right)$.

3-7: The square root of the derived reduced scattering coefficient perpendicular and parallel $\sqrt{\mu_{s, 90}^{\prime} / \mu_{s, 0}^{\prime}}$ for three different cylinder radii: $0.1,0.25$, and $1.5 \mu \mathrm{m}$ as a function of the fitted axes ratio $\beta$ of spatial resolved measurements.

4-1: (a) Sample spatial resolved reflectance image obtained in anisotropic medium. The cylinder was aligned along the $y$-axis. The image size is $1 \times 1 \mathrm{~cm}^{2}$. (b) The time resolved measurement parallel $\left(0^{\circ}\right)$ and perpendicular $\left(90^{\circ}\right)$ to the fiber direction (solid lines) at $\rho=0.975 \mathrm{~cm}$ and the reflectance predicted by (a) $R_{I D E-Z B C}$, (b) $R_{I D E-E B C}$, (c) $R_{A D E-Z B C}$, and (d) $R_{A D E-E B C}$. The optical properties used in the MC are $\mu_{s, c}^{\prime}=5.0 \mathrm{~cm}^{-1}, \mu_{a}=0.1 \mathrm{~cm}^{-1}, g=0.8, \mu_{s, b}^{\prime}=$ $8.0 \mathrm{~cm}^{-1}$, and $r=0.1 \mu \mathrm{m}$.

4-2: The derived reduced scattering coefficient perpendicular to the cylinder direction from using (a) $R_{I D E-Z B C}$, (b) $R_{I D E-E B C}$, (c) $R_{A D E-Z B C}$, and (d) $R_{A D E-E B C}$ for different cylinder radii: $0.1,0.25,1.5 \mu \mathrm{m}$ with different total reduced scattering coefficients $\mu_{s, t}^{\prime}$. The dotted lines indicate the theoretical values. Error bars represent the $95 \%$ c.i. of the fitted values. In here, the symbol is larger than the error bars.

4-3: The derived reduced scattering coefficients parallel to the cylinder direction are shown using (a) $R_{I D E-Z B C}$, (b) $R_{I D E-E B C}$, (c) $R_{A D E-Z B C}$, and (d) $R_{A D E-E B C}$ for different cylinder radii: $0.1,0.25$, and $1.5 \mu \mathrm{m}$ with different total reduced scattering coefficients $\mu_{s, t}^{\prime}$. The dotted lines indicate the theoretical values. Error bars represent the $95 \%$ c.i. of the fitted values.

4-4: The derived absorption coefficient from using (a) $R_{I D E-Z B C},(\mathrm{~b}) R_{I D E-E B C}$, (c) $R_{A D E-}$ ${ }_{Z B C}$, and (d) $R_{A D E-E B C}$ for different cylinder radii: $0.1,0.25$, and $1.5 \mu \mathrm{m}$ with different total reduced scattering coefficients $\mu_{s, t}$. The dotted lines indicate the theoretical values. Error bars represent the $95 \%$ c.i. of the fitted values.

4-5: The corrected reduced scattering coefficients associated with the cylinder $\mu_{s, c}^{\prime}$ obtained by the different models: (a) $R_{I D E-Z B C}$, (b) $R_{I D E-E B C}$, (c) $R_{A D E-Z B C}$, and (d) $R_{A D E-E B C}$ using the Eq. (5-6) compared with the true cylinder reduced scattering coefficients $\mu_{s, c}^{\prime}\left(90^{\circ}\right)$. The dotted lines indicate the theoretical values. Error bars represent the $95 \%$ c.i. of the fitted values

4-6: The square root of the derived reduced scattering coefficient perpendicular and parallel $\sqrt{\mu_{s, 90}^{\prime} / \mu_{s, 0}^{\prime}}$ using different models: (a) $R_{I D E-Z B C}$, (b) $R_{I D E-E B C}$, (c) $R_{A D E-}$ $Z B C$, and (d) $R_{A D E-E B C}$ for three different cylinder radii: 0.1, 0.25, and $1.5 \mu \mathrm{m}$ as a function of the fitted axes ratio $\beta$ of spatial-resolved reflectance measurements. 
5-1: This schematic diagram of the fiber optic Mach-Zehnder low coherence interferometer shows. SLD, superluminescent diode; PL, pilot light for aiming; PD, balance photo-detector; AF, amplifier \& band-pass filter; and DAQ, data acquisition board.

5-2: The reference intensity for the reference arm was recorded at different path-length arm distances, which is measured by a photo-detector (2011-FC, New Focus Inc., USA).

5-3: Examples of raw data measured at $0.1 \mathrm{~cm}$ away from incidence; (b) the mean path-length as a function of measurement distance; (c) the total reflectance and (d) the transition distance as a function of the mean path-length in tendon.

5-4: (a) Examples of raw data measured at $0.1 \mathrm{~cm}$ from incidence; (b) the mean pathlength as function of measurement distance; (c) the total reflectance and (d) the transition distance as function of the mean path-length obtained in skeletal muscle.

5-5: The mean path-length (a) and total reflectance (b) after transforming the y-axis by $y^{\prime}=2.43 y$ for the same tendon data shown in Fig. 5-2(b) \& (c), respectively.

5-6: The mean path-length (a) and total reflectance (b) after transforming the $y$-axis by $y^{\prime}=2.03 y$ for the same muscle data shown in Fig. 5-3(b) \& (c), respectively. 82

5-7: (a) The mean path-length and (b) total reflectance as function of measurement distance obtained in tendon. Also note the mean path-length (c) and total reflectance (d) after transforming the $y$-axis by $y^{\prime}=1.71 y$.

5-8: (a) The mean path-length and (b) total reflectance as function of measurement distance obtained in tendon. Also note the mean path-length (c) and total reflectance (d) after transforming the $y$-axis by $y^{\prime}=2.00 y$.

5-9: (a) The mean path-length and (b) total reflectance as function of measurement distance obtained in tendon. Also note the mean path-length (c) and total reflectance (d) after transforming the $y$-axis by $y^{\prime}=1.42 y$.

5-10: (a) The mean path-length and (b) total reflectance as function of measurement distance obtained in skeletal muscle. Also note the mean path-length (c) and total reflectance (d) after transforming the $y$-axis by $y^{\prime}=2.43 y$.

5-11: (a) The mean path-length and (b) total reflectance as function of measurement distance obtained in skeletal muscle. Also note the mean path-length (c) and total reflectance (d) after transforming the $y$-axis by $y^{\prime}=2.43 y$.

5-12: (a) The mean path-length and (b) total reflectance as function of measurement distance obtained in skeletal muscle. Also note the mean path-length (c) and total reflectance (d) after transforming the $y$-axis by $y^{\prime}=1.94 y$. 
5-13: (a) The mean path-length and (b) total reflectance as function of measurement distance obtained in skeletal muscle. Also note the mean path-length (c) and total reflectance (d) after transforming the $y$-axis by $y^{\prime}=2.62 y$.

5-14: (a) The mean path-length and (b) total reflectance as function of measurement distance obtained in skeletal muscle. Also note the mean path-length (c) and total reflectance (d) after transforming the $y$-axis by $y^{\prime}=1.19 y$.

5-15: (a) The mean path-length and (b) total reflectance as function of measurement distance obtained in skeletal muscle. Also note the mean path-length (c) and total reflectance (d) after transforming the $y$-axis by $y^{\prime}=2.54 y$.

6-1: Example raw optical path-length resolved reflectance obtained in intralipid $(20 \%)$

6-2: (a) Sample fitting results for $20 \%$ intralipid optical path-length measured (circles) at distance $0.0,0.05,0.1,0.15$, and $0.2 \mathrm{~cm}$ using Eq. (6-1). (b) The determined reduced scattering $\mu_{s}^{\prime}$ and absorption $\mu_{a}$ coefficients for measurement at distance from 0.0 to $0.2 \mathrm{~cm}$. The detection angle $\phi$ is $60^{\circ}$. Error bars represent the $95 \%$ c.i. of the fitted values.

6-3: The determined (a) reduced scattering and (b) absorption coefficients versus the calculated reduced scattering coefficient $\mu_{\text {'s }}$ for different intralipid solutions $20 \%, 15 \%, 10 \%$, and $8 \%$ at different measurement distances. (c) the determined reduced scattering and absorption coefficients for solutions with different intralipid concentrations at specific distance, $d=0.15 \mathrm{~cm}$. The detection angle $\phi$ is $60^{\circ}$. The dotted lines indicate the linear regression for the data. Error bars represent the $95 \%$ c.i. of the fitted values.

6-4: The determined (a) reduced scattering $\mu_{s}^{\prime}$ and (b) absorption $\mu_{a}$ coefficient versus the theoretical absorption coefficient for $15 \%$ intralipid solutions with different India ink $0.0 \%, 1.0 \%$, and $2.0 \%$ (c) the determined reduced scattering and absorption coefficient for different intralipid solution at $d=$ $0.2 \mathrm{~cm}$. The detection angle $\phi$ is $60^{\circ}$. The dotted lines indicate the linear regression for the data. Error bars represent the $95 \%$ c.i. of the fitted values.

6-5: (a) The raw optical path-length resolved reflectance profile of chicken liver. (b) The retrieved reduced scattering $\mu^{\prime}$ s and absorption $\mu_{a}$ coefficient at different measurement distances, $d=0.0,0.05,0.10,0.15$, and $0.20 \mathrm{~cm}$. Error bars represent the $95 \%$ c.i. of the fitted values.

6-6: Path-length resolved reflectance measured in tendon and the corresponding diffuse fitting at measurement distance $d$ of (a) $0.05 \mathrm{~cm}$ and (b) $0.10 \mathrm{~cm}$, respectively. The retrieved (c) reduced scattering $\mu_{s}^{\prime}\left(\mathrm{cm}^{-1}\right)$ and (d) absorption $\mu_{a}\left(\mathrm{~cm}^{-1}\right)$ coefficients at different distances $d=0.00,0.05,0.10$ and $0.15 \mathrm{~cm}$ and angular position $\alpha 0^{\circ}, 22.5^{\circ}, 45^{\circ}$, and $67.5^{\circ}$, and $90^{\circ}$. Error bars represent the $95 \%$ c.i. of the fitted values. 


\title{
Characterizing Optical Properties in Fibrous Tissues
}

\author{
Ali Shuaib \\ Dr. Gang Yao, Dissertation Supervisor
}

\begin{abstract}
Optical methods are promising for non-invasive tissue characterization. Tissue mechanical properties depend on structural properties of tissue constituents such as fiber alignment, size and concentration in fibrous tissues. Because the same structural properties also affect light propagation, optical properties obtained may be used to indicate tissue mechanical properties and can be applied to monitor earlier tissue structural changes.
\end{abstract}

Biological tissues can be classified into isotropic tissues and anisotropic tissues. The optical properties of isotropic tissues such as adipose tissue are independent of measurement direction. However, optical properties of anisotropic tissues such as tendon are different along different measurement directions. Although optical measurement in isotropic tissues is established and widely applied, light propagation in anisotropic tissues is not well understood.

A Monte Carlo model was applied to simulate light propagation in a fibrous tissue which was modeled as a mixture of aligned infinity long cylinders and randomly distributed background spherical particles. Both spatial- and time-resolved reflectance measurements were simulated. The spatial resolved reflectance profiles were quantitatively studied by using a nonlinear parametric fitting. The results indicated that 
the anisotropic diffuse theory can correctly calculate the surface reflectance in samples with fibers of small sizes. For larger fiber sizes, a correct coefficient needs to be applied.

Time-resolved reflectance measurements were applied to measure optical scattering and absorption properties in fibrous tissue. The performance of isotropic and anisotropic diffuse models was compared. In order to determine optical properties in fibrous tissue, the isotropic model needs two measurements that are parallel and perpendicular to the fibers. On the other hand, the anisotropic model only needs one measurement that is perpendicular to the fiber orientation. Both models can derive the background optical properties. The reduced scattering coefficient of the fiber component can be determined if the fiber size is known. The isotropic diffuse model with extrapolated boundary condition performed better than the other models in retrieving the reduced scattering coefficients of the cylinder and background.

To experimentally measure time-resolved reflectance, a fiber optics based lowcoherence Mach-Zehnder interferometer was developed. A low coherence superluminescent diode (SLD) was used as the light source to provide temporal resolution of $74.7 f s$ in air. A translational stage was used in the reference arm to provide over $2 \mathrm{~cm}$ pathlength scanning in sample. By changing the sample arm configuration, path-length resolved reflectance measurements were obtained at different distances from the incidence and different angles from fiber orientation. A time-resolved isotropic diffusion model was applied to retrieve optical properties by fitting the measured pathlength resolved reflectance. The system was calibrated in isotropic samples with known optical properties. The raw path-length resolved reflectance as well as the fitted reduced scattering coefficients showed strong angular dependency in anisotropic tissues. 
Anisotropic diffuse theory can satisfactorily describe the observed results in fibrous tissues. 


\section{CHAPTER 1}

\section{INTRODUCTION AND BACKGROUND}

The field of biomedical optics has become an important area of research due to many advantages of optical detection, such as non-invasiveness, non-toxicity and high speed. Optical techniques have been used in early diagnosis of many diseases, such as premalignant lesion and early neoplastic change (Backman et al. 1999; Mourant et al. 2009; Perelman et al. 1998; Wax and Pyhtila 2008). Light propagation in tissue depends on the scattering and absorption properties of the tissue components: cells, cell organelles, and various fiber structures (Minet et al. 1998; Muller et al. 1993; Muller and Roggan 1995; Tuchin 1994, 2002; Vo-Dinh 2003).

Light absorption is due to the various chromophores in the tissue. The absorption coefficient $\mu_{a}$ is defined as the probability that light is absorbed within an infinite small distance (Wang and $\mathrm{Wu}$ 2007). The overall absorption coefficient is the summation of contribution from each individual chromophore in tissue. In biological tissues, the major absorbers include water, hemoglobin, myoglobin and melanin. Due to the fact that absorption is wavelength dependent, it has been widely used in measuring the chemical concentration in various tissues. For example, tissue oxygenation is a valuable tool in assessment of hypoxia since the status of a tumor can show oxygen deficiency (Vishwanath et al. 2009). Usually, the wavelength used is in the near infrared (NIR) range $(700 \sim 1500 \mathrm{~nm})$ which is called "the biological window" (Cheong et al. 1990). 
The absorption in this spectral window is at minimum from water and protein; therefore, the light penetration depth is longer in biological tissue.

Light scattering in biological tissue is due to refractive index mismatch in different subcomponents such as nuclei, mitochondria and connective tissues. The size of these scattering components affects the scattering process. As a result, scattering-based diagnostic methods are used to monitor morphological changes in the tissue (Wang et al. 2005). The solution of Maxwell's equation under specific boundary condition, the Mie theory, is widely used to study the light scattering by a single particle.

Two parameters are used to characterize light scattering in tissue: 1) the scattering coefficient $\mu_{s}$ which describes the probability that the light is scattered in a unit distance; 2) the scattering phase function which describes the probability of the light being scattered in a certain direction for each scattering event. For many applications, the effect of scattering phase function can be simplified as a single parameter: the anisotropy factor $g$, that is the average cosine function of each scattering angle weight by the corresponding probability $(\mathrm{g}=<\cos (\theta)>)$, where $\theta$ is the scattering angle. The scattering coefficient and anisotropic factor are wavelength dependent. Under diffusion approximation in highly scattering tissues, the two quantities are combined into the reduced scattering coefficient $\mu^{\prime}{ }_{s}=(1-\mathrm{g}) \mu_{s}$.

Scatters in biological tissues are often modeled with spherical particles that are similar to many cells, micro-organism, and blood particles (Schmitt and Kumar 1996; Tuchin 1994, 2002). Spherical particles are isotropic, i.e. its optical properties are independent of photon incident directions. A system of randomly distributed, noninteractive spherical particles is the simplest tissue model. However, fibrous tissues (such 
as tendon, cartilage, skin, muscle, myocardium, retinal nerve fiber layer, etc.) are composed mostly of micro-fibrils and/or microtubules with the usual radius of the cylindrical structure elements from $5-200 \mathrm{~nm}$ and length from 10-25 $\mu \mathrm{m}$ to a few millimeters (Schmitt and Kumar 1996; Tuchin 2002). The distribution of the cylindrical radius might have sharp or broad mono-dispersive distribution (Tuchin 2002; Vo-Dinh 2003). Fibrous tissues can be modeled as infinitely long cylinders to represent the fibers with the random spherical particles representing cells. The light propagation in such fibrous tissues is anisotropic because their optical properties depend on incident angle. For instance, Marquez et al. (1998) showed that deriving the optical properties of the chicken breast depends on the muscle fiber direction. Nickell et al. (2000) also found that this was true in skin. It is necessary to understand the effects of anisotropic structures on optical properties. Such knowledge will benefit the development of accurate optical methods for diagnostic and therapeutic applications.

In this study, we focused on studying light propagation in fibrous tissues, such as tendon, ligament, cartilage, and skin. A brief introduction of different models and light propagation in biological tissue is provided below.

\subsection{Light propagation in biological tissue}

\subsubsection{Isotropic diffuse theory}

Light propagation in turbid medium can be modeled analytically by the radiative transport equation (RTE) (Case and Zweifel 1967; Ishimaru 1978). Analytical solutions to the RTE that describe photon migration can be obtained only by introducing further 
approximations. One of the most popular models used in the field of tissue optics is diffusion approximation (Farrell et al. 1992; Keijzer et al. 1988; Lin et al. 1997). The diffuse approximation is only valid if the light distribution can be considered as diffuse (Haskell et al. 1994; Patterson et al. 1989). The requirement for the validity of the diffuse approximation; therefore, are the reduced scattering coefficient must be much larger than the absorption coefficient $\left(\mu_{s}^{\prime} \gg>\mu_{a}\right)$ and the distance between the incident light and the point of interest needs to be large $\left(\rho>>1 / \mu_{s}^{\prime}\right)$ (Ishimaru 1978). The first requirement is true for most biological tissues in the red and near-infrared wavelength region.

The diffusion equation is:

$$
\frac{1}{c} \frac{\partial \phi(\vec{r}, t)}{\partial t}-D \nabla^{2} \phi(\vec{r}, t)+\mu_{a} \phi(\vec{r}, t)=Q_{o}(\vec{r}, t)
$$

where $c$ is the light speed in the turbid medium. $\phi$ is the fluence rate, $\mu_{a}$ is the absorption coefficient, and $Q_{o}$ is the light source. $D$ is the diffusion coefficient given by

$$
D=\frac{1}{3 \mu_{t}^{\prime}}=\frac{1}{3\left(\mu_{a}+\mu_{s}^{\prime}\right)}
$$

where $\mu_{s}^{\prime}$ is the reduced scattering coefficient defined as $\mu_{s}(1-\mathrm{g}) . g$ is the anisotropic factor and $\mu_{s}$ is the reduced scattering coefficient (Patterson et al. 1989). The transport coefficient $1 / \mu_{t}^{\prime}$ is the mean distance travelled by a photon between interactions (absorption and scattering).

In practical applications, the photon fluence rate $\phi$ is usually not measured directly. The measureable quantity is the reflectance $R(\rho, t)$, i.e. numbers of photons reaching that specific surface per unit area per unit time at specific distance away from the incident light, $\rho$. The following formula, known as Fick's Law, enables one to 
calculate the reflectance $R(\rho, t)$ as follows:

$$
R(\rho, t)=-\left.D \nabla \phi(\rho, z, t) \cdot(-\hat{z})\right|_{z=0}
$$

where $z$ is the distance normal to the boundary.

Time-resolved diffuse reflectance can be used to measure the optical properties of the medium. A pencil beam short-pulsed light is incident normally onto the semi-infinite medium, and then a detector measures the local diffuse time-resolved reflectance. When the light enters the tissue, the light cannot be assumed to be an isotropic source on the surface. The incident light can be described as an isotropic source at a depth that is equal to the mean free path of isotropic medium $z_{o}=1 / \mu^{\prime}{ }_{s}$ (Farrell et al. 1992; Haskell et al. 1994; Patterson et al. 1989) as shown in Fig. 1-1. All incident photons are assumed to be initially scattered at this depth, which will not affect significantly the accuracy of the fluence rates far from the source. Under a simplified zero-boundary condition (ZBC), where there is no refractive index mismatch at the tissue-air interface, the diffuse solution is:

$$
R(\rho, t)=\frac{e^{-\mu_{a} c t} e^{-\frac{\rho^{2}}{4 D c t}}}{2(4 \pi D c)^{3 / 2}(t)^{5 / 2}} z_{o} e^{\frac{-z_{o}^{2}}{4 D c t}}
$$

where $\rho$ is the measurement distance from the incident location, and $z_{o}=1 /\left(\mu_{a}+\mu_{s}^{\prime}\right)$ is the transport mean free path.

Furthermore, an appropriate boundary condition is needed if there is refractive index mismatch between the tissue and surrounding medium. The boundary condition should be that there is no photon flux from the surrounding medium back into the tissue. 
It has been shown that setting the fluence rate to zero at an extrapolate boundary outside the real boundary is a convenient way of approximately fulfilling the boundary condition for a medium-tissue interface (Haskell et al. 1994). The boundary is located at distance $z_{b}$ above the surface, where $z_{b}$ depends on the tissue diffusion coefficient $D$ and the Fresnel reflection at the surface. Therefore, this solution is called the extrapolated boundary condition $(\mathrm{EBC})$ that accounts for tissue-medium interface by adding a negative image source outside the tissue at distance $2 z_{b}+z_{o}$ above the surface as shown in Fig. 1-1; therefore, the time-resolved fluence rate is written as:

$$
\phi(d, z, t)=\frac{c e^{-\mu_{a} c t} e^{-\frac{\rho^{2}}{4 D c t}}}{2(4 \pi D c t)^{3 / 2}}\left[z_{o} e^{\frac{-z_{o}^{2}}{4 D c t}}+\left(z_{o}+2 z_{b}\right) e^{\frac{-\left(z_{o}+2 z_{b}\right)^{2}}{4 D c t}}\right]
$$

The reflectance is traditionally calculated by considering the flux across the boundary, which yields

$$
R(d, t)=\frac{e^{-\mu_{a} c t} e^{-\frac{\rho^{2}}{4 D c t}}}{2(4 \pi D c)^{3 / 2}(t)^{5 / 2}}\left[z_{o} e^{\frac{-z_{o}^{2}}{4 D c t}}+\left(z_{o}+2 z_{b}\right) e^{\frac{-\left(z_{o}+2 z_{b}\right)^{2}}{4 D c t}}\right]
$$

where $z_{b}=2 A D$, and $A=\left(1+R_{e f f}\right) /\left(1-R_{e f f}\right)$; where $R_{e f f}$ is the reflection of photon that are internally diffusely reflected at the boundary (Haskell et al. 1994).

Kienle and Patterson (1997) introduced an improved expression for the reflectance by taking the integral of the radiance over the background hemisphere. In addition, Kienle et al. (1998) derived a solution for two-layer geometry where the upper layer has a finite thickness, and the lower layer is infinite. Analytical solution for 
embedded sphere has also been derived. A comprehensive treatment on various analytical solutions of the diffusion equation is also presented in this paper (Haskell et al. 1994). Additional information on the influence of boundary conditions on the diffuse equation was analyzed by Hielscher et al. (1995, 1996).

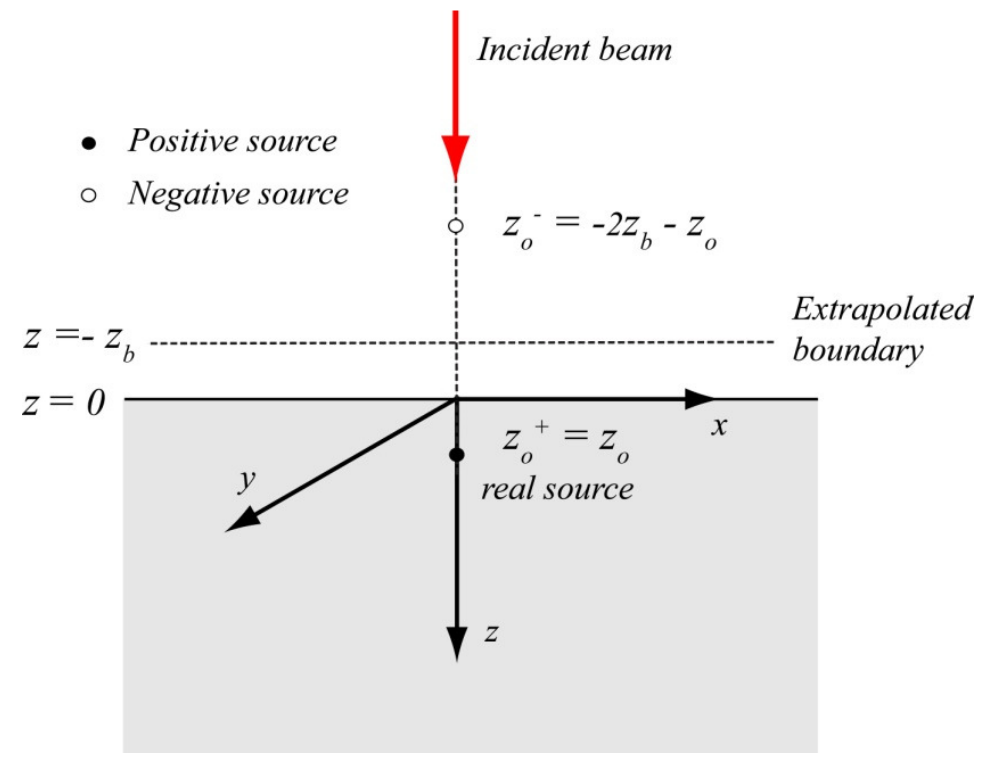

Figure 1-1: Geometry for the calculation of $R(\rho, \mathrm{t})$ for a semi-infinite isotropic medium. The incident beam is assumed to create an isotropic photon source at depth $z_{o}$, indicated by the close circle. The boundary condition can be met by adding a negative source indicated by the open circle.

\subsubsection{Anisotropic models}

The validity of applying the isotropic diffuse model is questionable in anisotropic tissues (Johnson et al. 2002; Kao et al. 1996; Kienle 2007). Recent studies modified the conventional isotropic diffuse equation (Heino et al. 2003; Stark and Lubensky 1996) by replacing the scalar diffusion coefficient with a diffusion tensor to describe the 
anisotropic tissue optical properties. The $D$ in Equation (1-3) becomes a diffuse tensor with non-zero diagonal components along three axes.

$$
\begin{gathered}
D=\left(\begin{array}{ccc}
D_{x} & 0 & 0 \\
0 & D_{y} & 0 \\
0 & 0 & D_{z}
\end{array}\right) \\
\frac{1}{c} \frac{\partial \Phi(r, t)}{\partial t}-\left(D_{x} \frac{\partial^{2}}{\partial x^{2}}+D_{y} \frac{\partial^{2}}{\partial y^{2}}+D_{z} \frac{\partial^{2}}{\partial z^{2}}\right) \Phi(r, t)+\mu_{a} \Phi(r, t)=Q_{0}(r, t),
\end{gathered}
$$

The equi-intensity of the surface reflectance is predicted to have elliptical shapes in a fibrous anisotropic medium with a highly organized structure (Johnson et al. 2002). At large distance, the ellipse is oriented parallel to the direction of the aligned fibers, along which the reduced scattering coefficient is smaller.

Kienle et al. (2004) was the first to use cylinders in a Monte Carlo model to simulate fibrous structures (Yousif and Boutros 1992). Kienle showed that the equiintensity has different orientations of ellipses at different distances from the incident light in dentin (Kienle et al. 2003). Several studies confirmed experimentally that the Monte Carlo results in several anisotropic media, such as porcine artery (Kienle et al. 2004), dentin (Kienle et al. 2003), and wood (Kienle et al. 2008). In addition, Kienle (2007) questioned the validity of the ADE theory based on his Monte Carlo simulation. Shuaib and Yao (2010) showed that the results from ADE theory are in agreement with Monte Carlo simulation when fiber size is small. 


\subsection{Isotropic and anisotropic biological tissues}

Several tissues, such as adipose and liver, are quite isotropic. Adipose tissue is a loose connective tissue that is composed of many adipocytes (Marieb and Hoehn 2008). Fat accounts for approximately $80 \%$ of the adipose tissue. The adipose tissue is usually located beneath the skin ("subcutaneous layer") and breast tissue. The major function of adipose tissue is to store the excess energy in the form of fat, whereas the minor functions are to provide mechanical protection and support around some major organs, and to reduce heat loss through skin (an insulation layer).

Some examples of anisotropic tissues are fibrous tissues such as tendon, ligament, cartilage, and skin, and skeletal muscle tissue. Fibrous tissues contain collagen fibers and can be found throughout the body. The three main components of fibrous tissues are cells, fibers, and extracellular matrix. The fibrous tissues' major function is to protect the organ, provide structural framework for the body, and connect body tissues with each other. In addition, skeletal muscle consists of thousand of muscle fibers, where the fibers are bound together by connective tissue. The main function of the skeletal muscle is to produce skeletal movement, maintain posture and body position, guard entrance and exit, and maintain body temperature. The anisotropic tissues will be discussed in more depth in the following sections.

\subsubsection{Tendon and ligaments}

Tendons and ligaments are a form of connective tissues that are subjective to mechanical forces in the body (Lieber 2002). Tendons connect muscle to bone and ligaments connect bone to bone. Tendon and ligaments (Figure 1-2(a)) are assembled 
from fiber bundles, or sometimes, fascicles. Fibers (Figure 1-2 (b) and (c) are formed from fibrils. Fibrils are formed from collagens (Kastelic et al. 1978). Fibroblast, shown in Figure 1-2 (b) and (c), is the most common type of cells found in tendon. Fibroblasts are responsible for synthesis and turnover of tendon fibers and ground substance (Lieber 2002).

Collagen is a fibrous protein that has a rod-like shape and is insoluble in water. Collagen is an abundant protein in the body that provides structural support for many tissues (Lieber 2002). Different collagen types are present. For instance, type I collagen is the most abundant collagen in the body. Type I collagen has a strong structure and is found in bone, tendon, and dentine. Type III collagen has a minimal presence in bone and tendon; even though it has similar distribution to Type I collagen.
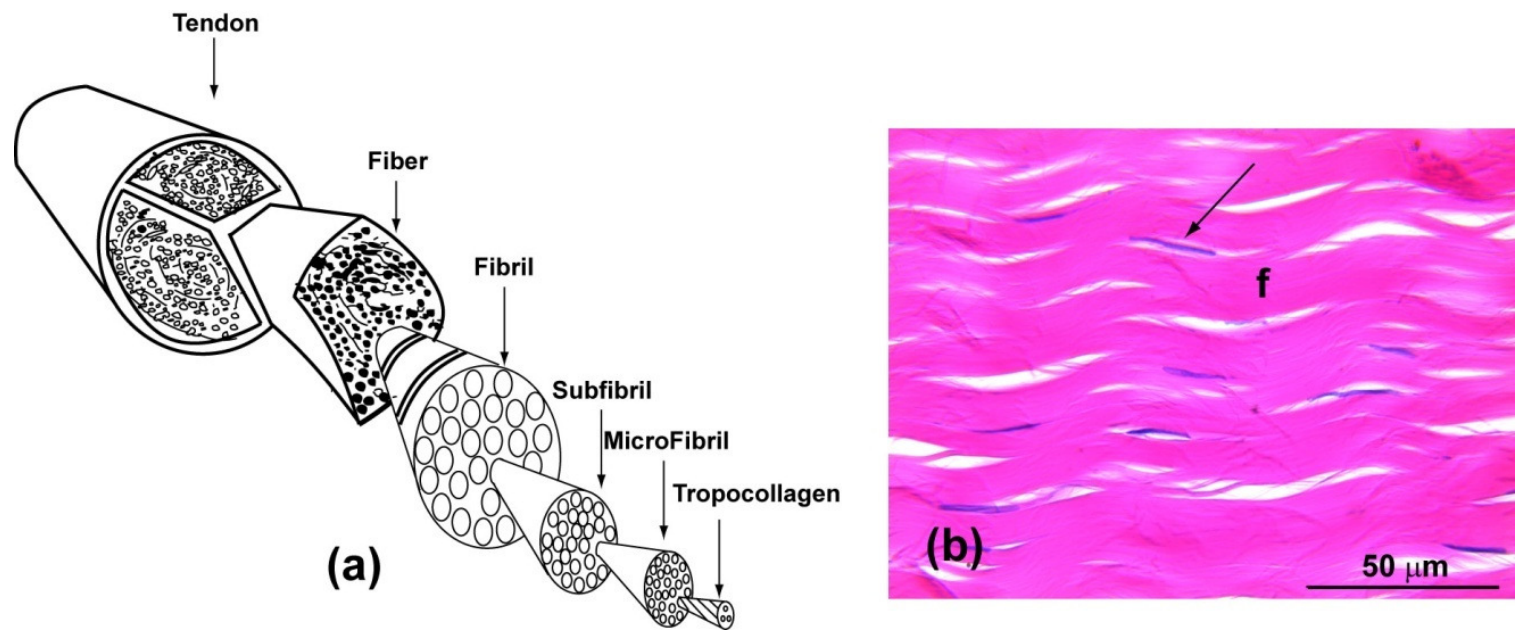

Figure 1-2: (a) Hierarchical structure of tendon. A tendon consists primarily of collagen. The illustration is sketched from (Kastelic et al. 1978). Light microscopy of a tendon in (b) longitudinal section. Regularly arranged fibers "f" are mixed with fibroblasts (arrow). Hematoxylin-Eosin (H\&E) stain magnified 40 times of its original size. 
For collagen to handle the mechanical force it experiences, it is arranged in a specific pattern. Even though the fibers are somewhat flexible, they have a great resistance to pulling force. Muscle-tendon units connect to bones, which often stabilize or move under considerable amounts of force (Lieber 2002). Ligaments are similar to tendons except that the bundles are irregularly arranged (Lieber 2002). This is likely due to the tendon being able to withstand strong force in one direction; however, ligaments need to be able to withstand force from a variety of directions in order to protect the joint. A ligament typically stretches across joints and helps to minimize excessive movements in the joint. In general, ligaments are unable to maintain control of the joint if weakness or failure of the muscle occurs, and damage to the ligament or joint will occur (Lieber 2002).

Several researchers have studied the morphological structure effect on the mechanical structure of tendons and ligaments. The distribution of collagen fibril diameters and the mean fibril diameter of connective tissues have been shown to depend on many different influences. Collagen fibril population changes during maturation (Frank et al. 1989), immobilization (Newton et al. 1990), and increased stress levels on the tissue (Michna 1984; Williams et al. 1985). In the connective tissue, the mean collaged fiber diameter has been suggested to be correlated with the resistance to applied maximum mechanical stress (Parry and Craig 1984).

Tendon and ligament abnormalities can lead to significant morbidity, including pain, rupture, and tear (Khan et al. 2000; Movin et al. 1997). For instance, achilles and ACL rupture have been linked to abnormalities of collagen when there is a degeneration of collagen or when collagen fiber becomes non-parallel or shows an irregular bundle 
dimension. In addition, collagen fiber size distribution between the healed lesion area of a ruptured tendon in comparison with normal tendons were found to be quite different (Matthew and Moore 1991).

\subsubsection{Articular cartilage structure}

Articular cartilage is located on the surface of the joints. The function of the articular cartilage is to absorb shocks and distribute the load to the bones. The mechanical properties of the cartilage are due to the contribution of water, collagen, and proteoglycan (PG). Water accounts for $70 \%$ of cartilge. Collagen and proteoglycan accounts for $50 \%$ and $10 \%$ of the cartilage dry weight, respectively (Tyler et al. 1995). Articular cartilage is composed mainly of Type II collagen fibers. Type I collagen fiber, the major type in the body, is present in very small percentages of the articular cartilage. The mechanical tensile of the cartilage is related to the collagen fibers' concentation and their arrangment

in the tissue; whereas the elastic resistance to compression is believed to be due to the high water content in the cartilage. 


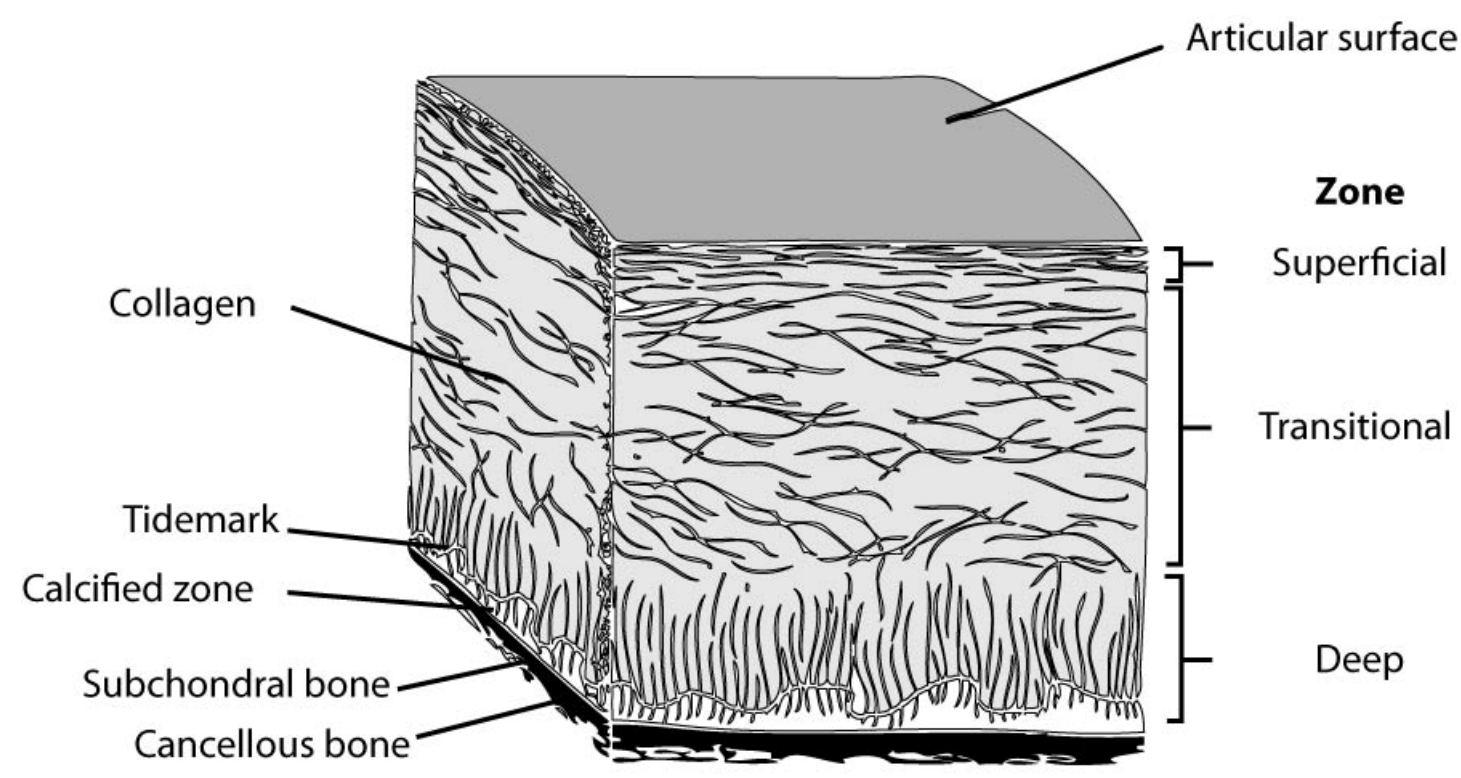

Figure 1-3: The illustration, showing collagen organization in the articular cartilage, is sketched from (Jazrawi et al. 2011).

The specific arrangement of the collagen fiber are different and depend on the location and the person. Figure 1-3 shows an example of collagen organization in the Articular cartilage (Nueman 1998). The superficial zone, the lamina splenders, accounts for $5-10 \%$ of the total thickness of the cartilage (Jeffery et al. 1991). In the superficial zone, the orientation of collagen fibrils are parallel to the aricular surface. Afterward, the transitional zone accounts for the largest percentage of the total thickness of the cartilage. The transitional zone contains a combination of the tangential and radial fibers. Then appears the deep zone, where the fiber orientation is tangential to the surface. At the calcified zone, the deepest zone, it is suggested that the fiber orientaiton is rotated and interwined. 
One of most common joint disease is osteoarthritis (OA), and it is the leading cause of disability in the United States. It is desirable to develop sensitive clinical diagnostic tools to identify and monitor the early degenerative processes of OA to aid in finding a cure or effective management to improve the quality of the life of the aging population. The disorder affects the knee, hip, vertebrae, and finger joints. Morphological changes happen in all tissues with the osteoarthritis joint; however, cartilage degeneration is a major characteristic of the disease. At first, chemical changes occur in the cartilage, such as an increase in water content and a loss of orientation in the surface collagen fibers (Mankin and Thrasher 1975; Venn and Maroudas 1977). Then, cartilage changes include clefts, and fibrillation form; fibrous tissue appears with the subchondral bone, and blood vessels from subchondral bone penetrate into the articular cartilage.

\subsubsection{Skin}

Skin is the largest organ in the in the body and serves four critical functions which are to (1) protect the body against the outer environment, (2) prevent the excess loss or gain of heat, (3) receive and interpret information from surrounding stimuli, and (4) produce vitamin D. Figure 1-4 shows the two main layers of skin: the epidermis and the dermis. Another minor layer, the subcutaneous layer, is a fatty layer underneath the skin, the thickness of which varies between humans (Williams 2002).

First of all, the epidermis is the outermost layer. The layer is composed primarily of epithelial cells, more specifically keratinocytes. Keratinocytes produce a tough protein called "keratin" that is formed in nails and hair. The skin's mechanical properties and its ability to be waterproof are mainly due to keratin protein. Also, melanocytes are present 
in the epidermis. Melanocytes produce melanin, which give the skin its characteristic color and protects the body from ultraviolet irradiation (Williams 2002).

The second layer, the dermis is immediately underneath the epidermis. Normally, the dermis is about four times thicker than the epidermis (Ross et al. 2003; Williams 2002). The dermis is composed of fibrous tissue, specifically fibroblasts. The protein collagen and elastin are excreted from the fibroblast into the extracellular matrix. The dermis also consists of the glands, hair follicles, veins, and nerves. The collagen fibrils are important for the strength and elasticity of skin; whereas, the elastin gives the dermis its resting tension (Williams 2002).

Studies have shown variations in the concentration and structure changes in the mechanical properties of the skin. For instance, reduction in the collagen concentration causes the skin to be fragile and cause wrinkle formation which is observed in the elderly (Smith et al. 1962). Sunlight exposure reduces the level of the collagen precursor and cross-links (Yamauchi et al. 1991). Also, smoking has been suggested to be related to an increase in wrinkling due the alteration of the collagen structure (Kadunce et al. 1991). 


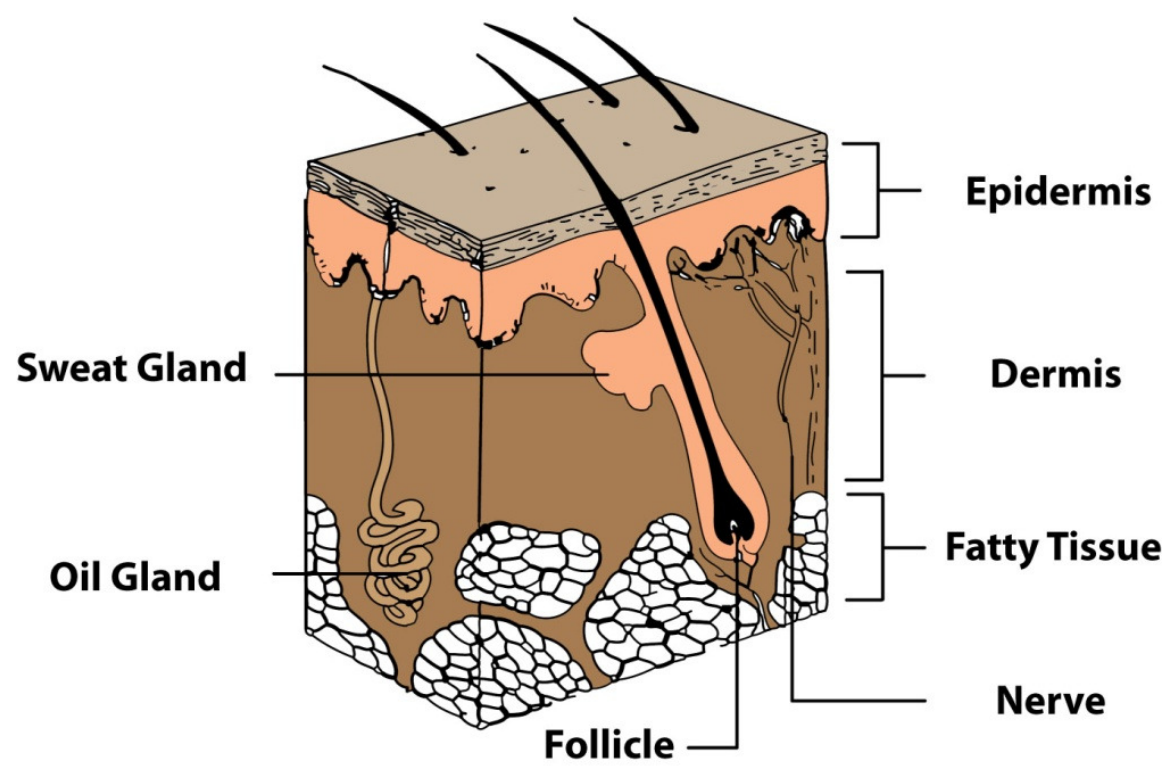

Figure 1-4: The anatomy of normal skin is shown here. The epidermis lies above the dermis, which lies above the subcutaneous fat or "fat cells". This illustration is sketched from (Victoria 2010).

\subsubsection{Skeletal muscle}

Skeletal muscle is the most abundant tissue and responsible for many important physiological functions (Lieber 2002). Whole muscle (Figure 1-5(a)) consists of collection of muscle fiber, fascicles, by an elaborate connective tissue matrix. In each single fiber muscle, there are hundreds of small myofibrils arranged in parallel. The diameter of the myofibrils is about $1 \mu m$ (Lieber 2002). In myofibrils, there are unique units know as sarcomeres that are arranged in series. A sarcomere is the functional unit of muscle contraction that is the responsible for mechanical and physiological functions (Bannemann and Laing 2004; Laing and Nowak 2005). Many skeletal muscle diseases eventually relate to the changes of the sarcomere protein (Laing and Nowak 2005). 

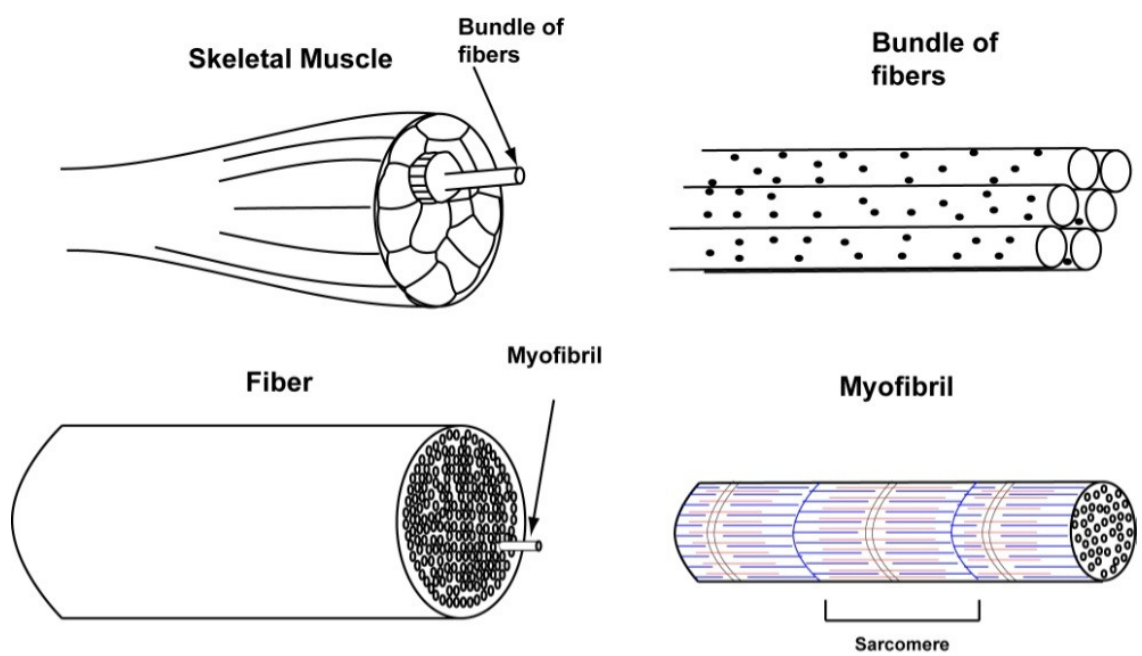

(a)

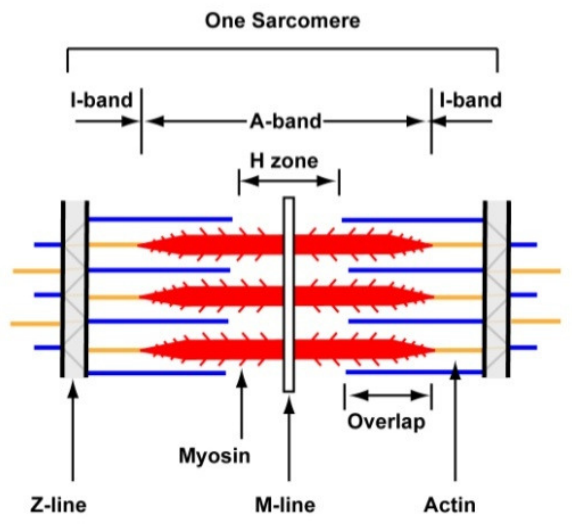

(b)

Figure 1-5: (a) The hierarchy of the skeletal muscle. (b) Illustration of sarcomere structure. These illustrations are sketched from (Lieber 2002).

A sarcomere appears striated mainly due to the alternating darker A-bands and lighter I-bands as shown in Figure 1-5(b). The $\mathrm{H}$ zone is the segment with only myosin proteins. The Z-line is located in the middle of the I-band, whereas the M-line is located in the middle of the $\mathrm{H}$ zone. The distance between the two Z-lines is usually defined as 
the sarcomere length. The A-band is the region where both the actin and myosin are present.

The majority of muscular diseases are related to the changes in sarcomere proteins (Proske and Morgan 2001). For instance, Duchenne Muscular Dystrophy (DMD) is one of most common diseases that affects children and is due to the lack of a protein product known as "dystrophine" (Lieber 2002). DMD is a destructive disease that strikes young boys in their first year of life. Most DMD patients use wheel chairs for mobility and experience muscle weakness by the age of about 13. By the age of 20, most DMD patients are dead. From the histological examination, their muscle has significant variation in fiber size and central nuclei (Watkins and Cullen 1982). In addition to the muscle fiber variations, the connective tissue and fat cells within the tissue proliferation lead to the muscle becoming non-functional.

\subsection{Objective of the study}

A variety of noninvasive or minimally invasive methods have been used for assessments in tissue. Biopsy, which is considered the "gold-standard" of diagnostic methods, has disadvantages. The location may be hazardous or difficult to obtain or it may produce a false positive if it misses the region that contains the disease. Also, Computed Tomography (CT) has been widely used. However, it causes radiation damage and lack contrast to evaluate early tissue changes (Chan et al. 1991). Magnetic resonance imaging (MRI) represents a noninvasive powerful tool for diagnostic joints abnormalities (Eckstein and Glaser 2004). However, due to the high cost, relatively low resolution, and 
the long time required for data acquisition, it has not achieved wide-spread use for routine detection.

An optical technique that is able to analyze the structure of the biological tissue could have four potential benefits for experimental purposes: 1) it is capable of on-line monitoring of the construction of tissue engineering; 2) it can be used to detect earlystage degenerative changes and it can be used to monitor early stages degenerative bone disease in a non-invasive or minimally invasive way; 3) because animals model are still used to develop and evaluate new drugs, optical techniques can provide their tissue measurements without killing the animal for dissection and tissue analysis. This could improve accuracy and reduce the cost of experiment.

\subsection{Outline}

This dissertation focuses on improving our understanding of light propagation in anisotropic tissue. Fibrous tissue, such as tendon, ligament, cartilage, and skin, were studied in detail using Monte Carlo simulation and experimental studies. In Chapter 2, Monte Carlo simulation was employed to simulate the propagation of light in a fibrous anisotropic scattering medium. A parametric equation was used to quantitatively characterize the geometric profiles of the reflectance patterns. The axes ratio of the reflectance of tissues with different cylinder sizes were measured and compared with the anisotropic diffuse theory (ADE). In Chapter 3, we investigated the possibility of retrieving optical properties in anisotropic fibrous tissue using time-resolved measurements generated from Monte Carlo simulation. An isotropic diffuse model was 
used to determine the optical properties from simulated time-resolved reflectance. We found that the isotropic diffuse model is not capable of describing the light propagation in fibrous tissue unless the fiber size is small. In Chapter 4, we further investigated the possibility of retrieving the fibrous tissue optical properties using isotropic and anisotropic diffuse models under two commonly used boundary conditions. Afterward, a comparison between the isotropic and anisotropic diffuse models retrieved optical properties and concluded which model was better in describing the light propagation in fibrous tissue.

In Chapter 5, a low-coherence Mach-Zehnder interferometer was developed to measure the path-length resolved optical reflectance in both isotropic turbid media and anisotropic tissues (tendon and skeletal muscles). In anisotropic tissue, the measured optical path-length depended strongly on the fiber orientation. The results revealed both similarities and differences in tendon and skeletal muscles which are likely caused by their different structures. These results provided useful evidence leading to a better understanding of photon migration in such complicated biological tissues. In Chapter 6, a time-resolved diffusion model was applied to retrieve the optical properties from the path-length resolved reflectance from anisotropic (tendon) tissue. The reduced scattering coefficient is strongly dependent on the fiber direction, whereas the absorption is independent of the fiber direction. Finally, Chapter 7 presents the summary and discusses potential future work. 


\section{CHAPTER 2}

\section{EQUI-INTENSITY DISTRUBTION OF OPTICAL REFLECTANCE IN FIBROUS TURBID MEDIUM ${ }^{1}$}

\subsection{Introduction}

Optical methods have been widely used to study biological tissues. In many studies, the tissue sample can be assumed to be isotropic, i.e., its optical properties are independent of photon incident directions. However, this is not true in some biological tissues such as muscle (Marquez et al. 1998), skin (Nickell et al. 2000), dentin (Forster et al. 2001), and white matter (Moseley et al. 1990), where anisotropic fibrous or tubular structures are abundant. For example, Marquez et al. (1998) found that optical properties of the chicken breast differed significantly when measured parallel or perpendicular to the muscle fiber orientation.

In order to describe light propagation in anisotropic tissue, the conventional isotropic diffuse equation can be modified (Heino et al. 2003; Stark and Lubensky 1996) by replacing the scalar diffusion coefficient with a diffusion tensor. This anisotropic diffuse equation (ADE) model predicts that the equi-intensity profiles of the surface reflectance have elliptical shapes (Johnson et al. 2002) in media with a predominant structural orientation (such as aligned fibers). The ellipse is elongated along the direction with a smaller reduced scattering coefficient. The ratio of the two primary axes of the

\footnotetext{
${ }^{1}$ The Chapter was adapted from a published paper: Shuaib, A. and G. Yao, "Equi-intensity distribution of optical reflectance in a fibrous turbid medium," App. Opt. 49, 838-844 (2010).
} 
ellipse is inversely proportional to the square root of the corresponding reduced scattering coefficients along the two axes.

In another approach, Dagdug et al. (2003) extended the continuous time random walk (CTRW) theory to analyze light propagation in anisotropic turbid medium by assigning different transition probabilities along different directions. In a fibrous sample, the model used a bias parameter $\mathrm{B}$, defined as the ratio of the transition probability parallel with and perpendicular to the fibers direction, to characterize the anisotropy. This model also predicts an elliptical pattern of the equi-intensity reflectance in fibrous tissues. The ratio of the two primary axes is proportional to the square root of the corresponding transitional probabilities.

The elliptical equi-intensity reflectance profile predicted from both ADE and CTRW models has been observed in several anisotropic tissue models (Hebden et al. 2004; Ranasinghesagara et al. 2006; Sviridov et al. 2005). Kienle et al. (2004) showed that the equi-intensity profiles had different elliptical orientations at different evaluation distances in dentin. Nickell et al. (2000) found that the elliptical reflectance profile in skin tissue was caused by the preferential orientation of the collagen fibers.

Kienle (2007) studied spatial- and time-resolved diffuse transmission in fibrous anisotropic media, and found the results obtained from ADE theory were very different from those obtained with Monte Carlo simulation. Kienle (2007) proposed that Monte Carlo models based on light scattering by cylindrical structures (Kienle et al. 2004) were more accurate for studying light propagation in fibrous scattering media. Schäfer and Kienle (2008) proved that this approach had a good agreement with Maxwell theory 
based solutions when the volume cylinder density is small $(\rho<10 \%)$. Several studies showed that the Monte Carlo simulation had good agreements with the experimental results obtained in porcine artery (Kienle et al. 2004), dentin (Kienle et al. 2003) and wood (Kienle et al. 2008).

Although discrepancies were noticed when comparing the equi-intensity profiles of the diffuse transmittance obtained by ADE and Monte Carlo simulation (Kienle 2007), these differences have not been studied in detail. In this study, we applied Monte Carlo simulations to investigate the equi-intensity profiles of the diffuse reflectance in an anisotropic medium composed of a mixture of cylinders and spheres scatters. A parametric equation was used to describe quantitatively the reflectance images. The effects of the background optical properties and the cylinder size were investigated. We found that the Monte Carlo results approached the predictions of ADE theory when the cylinders have a smaller radius. 


\subsection{Methods}

\subsubsection{Simulation model}

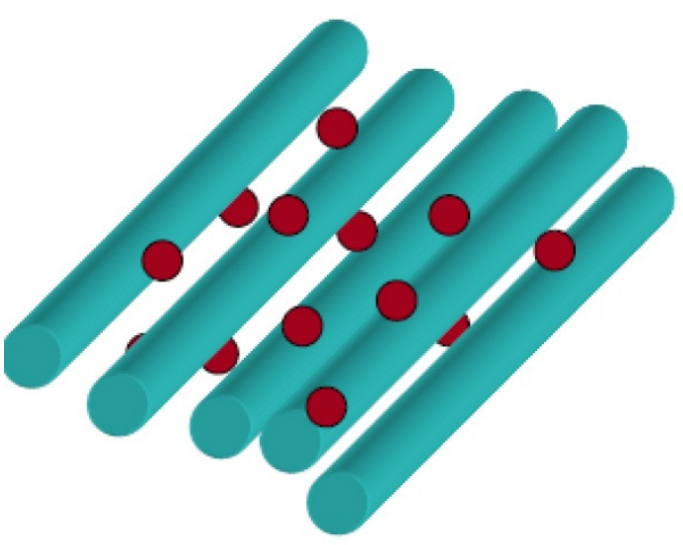

(a)
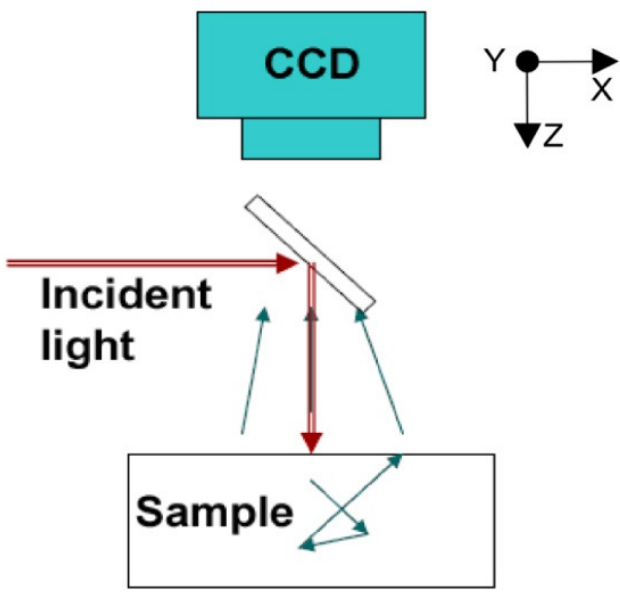

(b)

Figure 2-1: (a) Illustration of the scattering medium, which is composed of infinite long cylinders and uniformly distributed spherical particles. (b) Schematic diagram of the simulated experimental setup.

The scattering medium model used in this study has been previously described by Kienle et al. (2004) to simulate anisotropic fibrous tissues. As shown in Fig. 2-1(a), the sample was a semi-infinite medium composed by two different scattering particles: 1) organized infinite long cylinders and 2) randomly distributed background spherical particles. The cylinders were parallel to the sample surface. A single isotropic absorption coefficient $\mu_{\mathrm{a}}$ was assigned to the sample. An isotropic scattering coefficient $\mu_{\mathrm{s}, \mathrm{b}}$ and anisotropy $g_{\mathrm{b}}$ were assigned to the background spherical particles. The conventional Henyey-Greenstein function was used as the scattering phase function for these spherical particles. The scattering coefficient $\mu_{\mathrm{s}, \mathrm{c}}$ of the cylinders at an incident angle $\xi$ can be calculated as: 


$$
\mu_{s, c}(\xi)=2 \mathrm{r} * \mathrm{c}_{\mathrm{A}} * \mathrm{Q}_{\mathrm{s}}(\xi)
$$

where $r$ is the cylinder radius, $\mathrm{c}_{\mathrm{A}}$ is the concentration of cylinders $\left(\mathrm{mm}^{-2}\right)$, and $\mathrm{Q}_{\mathrm{s}}(\xi)$ is the scattering efficiency and can be calculated using the algorithm described by Yousif and Boutros (1992). The volume density of the cylinders can be calculated as $c_{A} \times \pi r^{2}$. The incident angle $\xi$ is defined as the angle formed by the incident light and the cylinder axis. $\xi=0^{\circ}$ indicates light incident along the cylinder; whereas $\xi=90^{\circ}$ indicates light incident perpendicularly to the cylinder.

The light scattering direction by a cylinder can be defined by the following two angles: the angle between the scattered light and the cylinder axis which is the same as the incident angle $\xi$ and the scattering angle $\phi$ defined as the angle between the incident and scattered light within a projection plane perpendicular to the cylinder. Therefore the scattered light is restricted to a cone with the cylinder as the axis and has a half-angle of $\xi$ (Bohern and Huffman 1983). As a conventional definition, the scattering angle $\phi$ is defined as the angle between the incident and scattering direction. The corresponding scattering anisotropy $g_{c}$ can be then derived as:

$$
g_{c}(\xi)=\langle\cos \theta\rangle=\cos ^{2} \xi+\sin ^{2} \xi \frac{\int_{0}^{\pi} \cos \phi p(\phi, \xi) \sin \phi d \phi}{\int_{0}^{\pi} p(\phi, \xi) \sin \phi d \phi}
$$

The scattering phase function $p(\phi, \xi)$ can be calculated using Yousif and Boutros's method (1992). When incident along the cylinder $\left(\xi=0^{\circ}\right)$, the light is not scattered and thus $\mathrm{g}_{\mathrm{c}}=1$. The reduced scattering coefficient of the cylinders is calculated as $\mu_{\mathrm{s}, \mathrm{c}}^{\prime}=\mu_{\mathrm{s}, \mathrm{c}}(1-$ $g_{\mathrm{c}}$ ). The total reduced scattering coefficient of the sample is $\mu_{\mathrm{s}}{ }^{\prime}=\mu_{\mathrm{s}, \mathrm{c}}{ }^{\prime}+\mu_{\mathrm{s}, \mathrm{b}}$, which is also a function of the incident angle $\xi$ 


\subsubsection{Monte-Carlo algorithm}

The simulated imaging setup is shown in Fig. 2-1(b). A pencil beam $(\lambda=800 \mathrm{~nm})$ was incident at $90^{\circ}$ upon the semi-infinite sample. The backscattered light at the sample surface was directly imaged by a CCD camera. The movement of a photon packet inside the sample was determined based on the local scattering and absorption coefficients (Wang et al. 1995). At each scattering event, a sampling method was applied to determine whether the photon was scattered by the cylinders or the background spherical particles:

$$
\text { if } \zeta<\frac{\mu_{s, c}}{\mu_{s, c}+\mu_{s, b}} \text {, scattered by cylinders }
$$

$$
\text { else, scattered by spherical particles }
$$

where $\zeta$ is a uniformly distributed random number between $[0,1]$. If the photon was scattered by spherical particles, the standard procedure (Wang et al. 1995) using the Henyey-Greenstein phase function was applied to determine the scattering direction. If the photon was scattered by cylinders, the new photon direction was determined by sampling the cylindrical scattering phase function $p(\phi, \xi)$. To expedite the computation, the cylindrical scattering phase function was pre-calculated at different incident and scattering angles at a $0.5^{\circ}$ precision and loaded into the Monte-Carlo program at the beginning of the simulation. To determine the scattering direction, the incident direction $\xi$ of a photon was used as an index to retrieve the stored cylinder phase function. If the phase function was not pre-calculated at this particular incident angle, linear interpolation was applied by using the phase functions calculated at the two closest angles to obtain the 
function at this particular angle. The new photon scattering angle $\xi$ was then sampled from the phase function $p(\phi, \xi)$ :

$$
\zeta \leq \frac{\sum_{\phi=0}^{\phi} p(\phi, \xi)}{\sum_{\phi=0}^{180} p(\phi, \xi)}
$$

where $\zeta$ is a uniformly distributed random number between $[0,1]$. The simulation process continued until a photon was completely absorbed in the medium or re-emitted out of the sample. The physical location and weight of the re-emitted photon were then stored in arrays to represent 2D images of the diffuse reflectance.

\subsubsection{Image processing}

The equi-intensity profiles were obtained by extracting all pixels with the same reflectance. Specifically, a pixel located at a specific distance from the incident point was selected and its intensity was set as a reference to find all pixels with the same intensity. A $\pm 1 \%$ error margin was applied in the search and the coordinates of all identified pixels were stored. Then the Levenberg-Marquardt algorithm was applied to obtain the best numerical fitting using the following parametric equation $f(x, y)$ (Ranasinghesagara and Yao 2007):

$$
f(x, y)=\left(\frac{|x|}{r_{x}}\right)^{q}+\left(\frac{|y|}{r_{y}}\right)^{q}-1=0
$$

where the $r_{\mathrm{x}}$ and $r_{y}$ represent the half axial length along the $x$ - and $y$-axis, respectively. In 
the simulation, the cylinders were always aligned with the $y$-axis. The shape parameter $q$ represents the geometrical shape of the equi-intensity profile with $q=1$ indicating a rhombus and $q=2$ indicating an ellipse. This parametric function can represent any geometric shapes between a perfect rhombus and a perfect ellipse (Ranasinghesagara and Yao 2007). The ratio of the two axes was calculated as:

$$
\beta=r_{\mathrm{y}} / r_{\mathrm{x}}
$$

\subsection{Results}
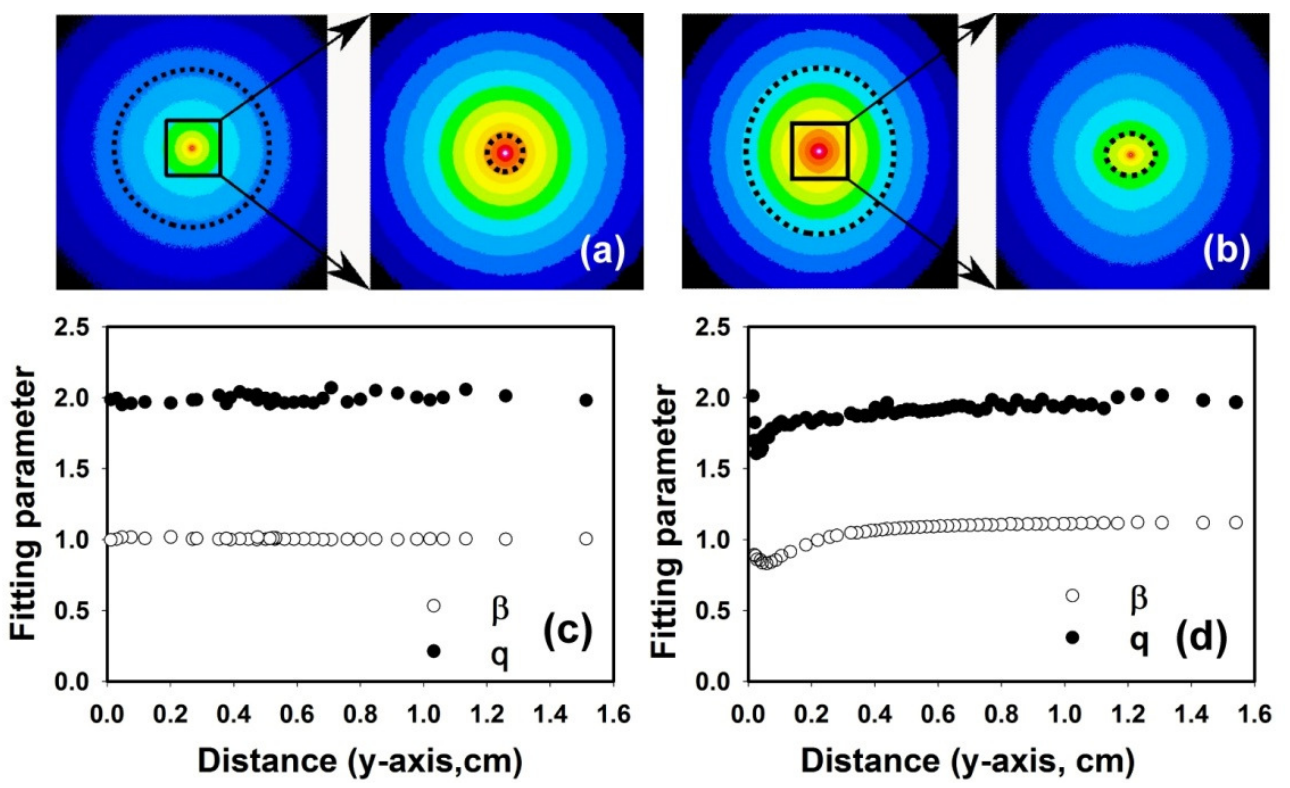

Figure 2-2: Sample reflectance images obtained in (a) isotropic and (b) anisotropic mediums. The cylinders were aligned with the y axis (vertical direction). The dash lines were sample fitting results using Eq. (5). The fitting parameters $\beta$ and $\mathrm{q}$ were shown as a function of the distance along the $\mathrm{y}$ axis in the above (c) isotropic and (d) anisotropic media.

Figure 2-2(a) shows an example of the reflectance image in an isotropic medium (without any embedded cylinders). The image was shown using a pseudo color map with 
different colors indicating different reflectance. The following optical properties were used for this isotropic medium: $\mu_{\mathrm{s}, \mathrm{b}}=30.0 \mathrm{~cm}^{-1}, g_{\mathrm{b}}=0.8$ and, $\mu_{\mathrm{a}}=0.01 \mathrm{~cm}^{-1}$. The simulated reflectance image had a pixel resolution of $100 \mu \mathrm{m}$ and a size of $5 \times 5 \mathrm{~cm}^{2}$. In order to have a better view of the reflectance distributions close to the incident point, images with a higher resolution of $10 \mu \mathrm{m}$ but a smaller size of $1 \times 1 \mathrm{~cm}^{2}$ are also shown. The dotted lines in the images were example fitting results using Eq. (5). The equiintensity distribution of the isotropic medium was a circle. Figure 2-2(c) shows the axes ratio $\beta$ and $q$ parameter obtained by fitting the corresponding spatial resolved reflectance images in Fig. 2-2(a). The fitted results were: axes ratio $\beta=1.0$ and $q \sim 2.0$, indicating a perfect circular profile.

As a comparison, Figure 2-2(b) shows a reflectance image obtained in an anisotropic medium where the cylindrical component had a radius of $r=1.5 \mu \mathrm{m}$, refractive index $n_{c}=1.46$ and a volume density of $\rho=0.98 \%$ and the background refractive index $n=1.36$. The corresponding scattering coefficient was $\mu_{\mathrm{s}, \mathrm{c}}=109.1 \mathrm{~cm}^{-1}$ and anisotropy $\mathrm{g}_{\mathrm{c}}=0.945$ both at incident angle of $\xi=90^{\circ}$. The background spherical scatters had $\mu_{\mathrm{s}, \mathrm{b}}=30.0 \mathrm{~cm}^{-1}$ and $g_{\mathrm{b}}=0.8$. The absorption coefficient was $\mu_{\mathrm{a}}=0.01 \mathrm{~cm}^{-1}$. The cylinders were aligned with the y-axis. As shown in Fig. 2-2(b), the equi-intensity profile of the reflectance image showed an ellipse perpendicular to cylinder at small distance from the incidence; whereas the ellipse became parallel to the cylinders at large distance. These observations were confirmed by the fitting results as shown in Fig. 22(d). The $q$ parameter at near distance had a value of $\sim 2.0$, indicating an ellipse. It decreased to $\sim 1.6$ at $\sim 0.04 \mathrm{~cm}$ and increased again until stabilizing at $\sim 2.0$. At the same time, the axes ratio $\beta$ decreased from 0.9 to 0.8 , increased thereafter and stabilized at 
Therefore, as the evaluation distance increased, the fitting parameters $\beta$ and $q$ went through a transition and stabilized as an ellipse along the cylinders at larger distances. To better describe this transition, a "transition distance" can be defined as the distance from the incident point where the fitted axes ratio reached $90 \%$ of the final stable value.

Figure 2-3 shows the stable fitted axes ratio $\beta$ obtained in anisotropic media with three different cylinder radii of $0.1,0.25$ and $1.5 \mu \mathrm{m}$. The $\beta$ results were plotted against the ADE predictions, i.e., the square root of $\mu_{s}^{\prime}(x) / \mu_{s}^{\prime}(y)$, where the $\mu_{s}^{\prime}(x)$ and $\mu_{s}^{\prime}(y)$ are the reduced scattering coefficient perpendicular and parallel to the cylinder, respectively. The dash line in Fig. 2-3(a) shows the prediction from ADE theory that the axes ratio $\beta=r_{\mathrm{y}} / r_{\mathrm{x}}$ is equal to $\sqrt{\mu_{s}^{\prime}(x) / \mu_{s}^{\prime}(y)}$. The background optical properties were maintained at $\mu_{\mathrm{s}, \mathrm{b}}=30 \mathrm{~cm}^{-1}, g_{\mathrm{b}}=0.8$, and $\mu_{\mathrm{a}}=0.01 \mathrm{~cm}^{-1}$. We altered $\sqrt{\mu_{s}^{\prime}(x) / \mu_{s}^{\prime}(y)}$ by changing the concentration of the cylinders $\left(c_{A}\right)$ in the medium. The cylinder volume densities $(\rho)$ in all samples ranged from $0.14 \%$ to $0.98 \%$. 


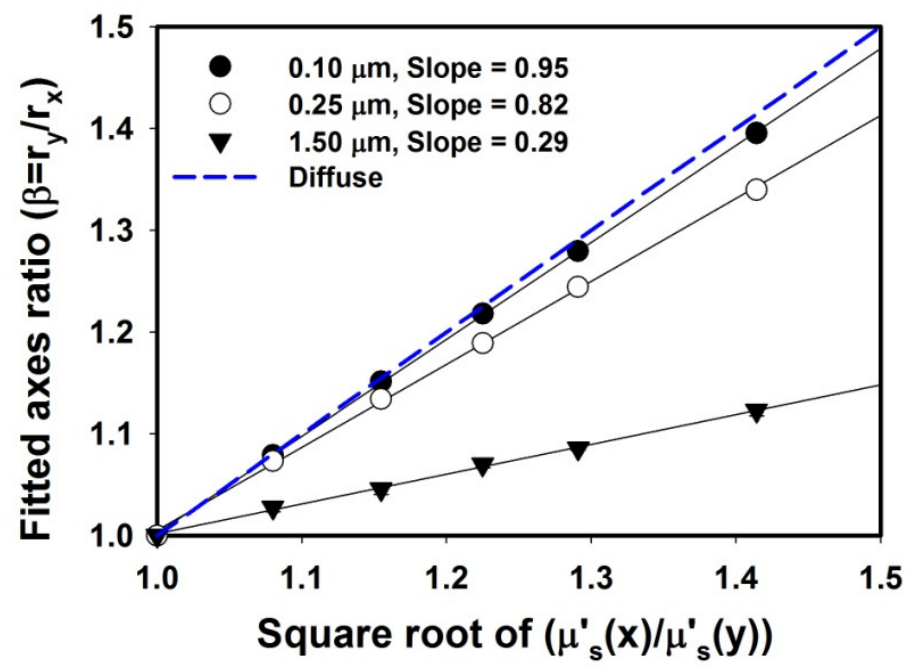

Figure 2-3: The fitted axes ratio $\beta$ versus the $\operatorname{ADE}$ prediction of $\sqrt{\mu_{s}^{\prime}(x) / \mu_{s}^{\prime}(y)}$ in anisotropic media of three different cylinder radii: $0.1,0.25$, and $1.5 \mu \mathrm{m}$. The background optical properties used in the simulation were $\mu_{\mathrm{s}, \mathrm{b}}=30 \mathrm{~cm}^{-1}, \mathrm{~g}_{\mathrm{b}}=0.8$, and $\mu_{\mathrm{a}}=0.01 \mathrm{~cm}^{-1}$.

A linear relationship was observed between $\sqrt{\mu_{s}^{\prime}(x) / \mu_{s}^{\prime}(y)}$ and the fitted axes ratio $\beta$ at all cylinder sizes. As shown in Fig. 2-3, all data points can be linearly fitted with $\mathrm{R}^{2}$ $\geq 0.99$. However, the fitted lines had different slopes: $0.95,0.82$ and 0.29 for cylinders with radius of $0.1,0.25$ and $1.5 \mu \mathrm{m}$, respectively. The point $(1,1)$ represents the results from isotropic samples. All regression lines in Fig. 2-3 passed through the point $(1,1)$ with a very small Standard Error of Intercept $(<0.015 \%)$. Interestingly, when the cylinder radius was small $(0.1 \mu \mathrm{m})$, the fitted axes ratio $\beta$ from the Monte Carlo simulations approached the prediction from the ADE theory.

To further confirm this, the fitted axes ratio $\beta$ was calculated at different cylinder radius from 0.05 to $1.5 \mu \mathrm{m}$ while $\sqrt{\mu_{s}^{\prime}(x) / \mu_{s}^{\prime}(y)}$ was maintained at constant value of 1.08 . The background optical properties were the same as before. The cylinder volume 
densities $(\rho)$ in all samples ranged from $0.16 \%$ to $0.29 \%$. As shown in Figure $2-4, \beta$ indeed approached the ADE predication when the cylinder radius was small.

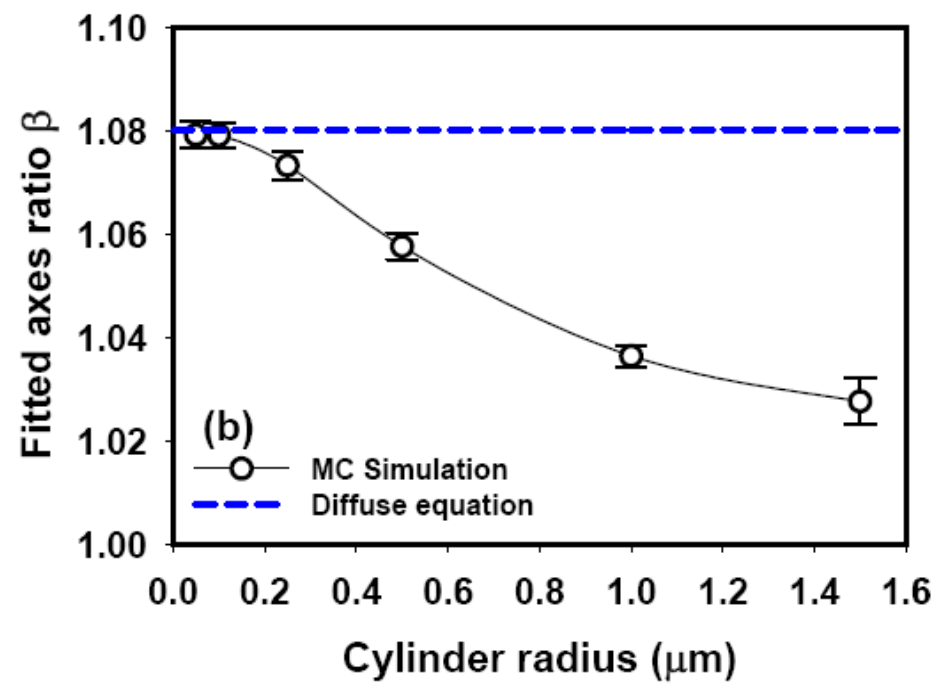

Figure 2-4: The fitted axes ratio $\beta$ versus cylinder radius. $\sqrt{\mu_{s}^{\prime}(x) / \mu_{s}^{\prime}(y)}$ was maintained at constant value of 1.08. The background optical properties used in the simulation were $\mu_{s, b}=30 \mathrm{~cm}^{-1}, \mathrm{~g}_{\mathrm{b}}=0.8$, and $\mu_{\mathrm{a}}=0.01 \mathrm{~cm}^{-1}$.

A small variation in the cylinder refractive index $n_{c}$ had little effect on the fitted axis ratio $\beta$. Using cylinders with a radius of $r=0.1 \mu \mathrm{m}$, the calculated $\beta$ had less than $0.5 \%$ variation when the cylinder refractive index was changed from 1.44 to 1.48 . In the above calculation, the background optical properties were maintained at $\mu_{\mathrm{s}, \mathrm{b}}=30 \mathrm{~cm}^{-1}, g_{\mathrm{b}}$ $=0.8, \mu_{\mathrm{a}}=0.01 \mathrm{~cm}^{-1}$. The $\sqrt{\mu_{s}^{\prime}(x) / \mu_{s}^{\prime}(y)}$ was maintained at a constant value of 1.41. 


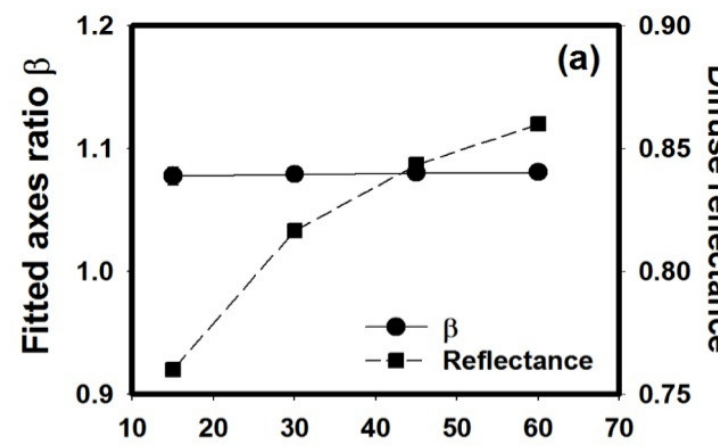

Background scattering $\mu_{\mathbf{s}, \mathbf{b}}\left(\mathrm{cm}^{-1}\right)$

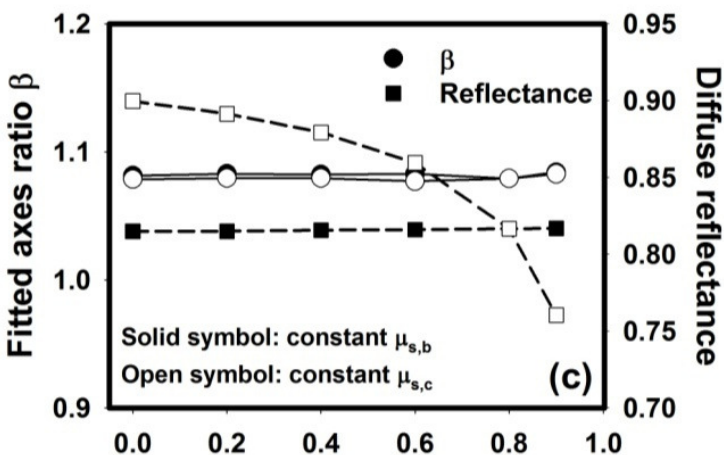

Backgroun anisotropy $\mathbf{g}_{\mathbf{h}}$

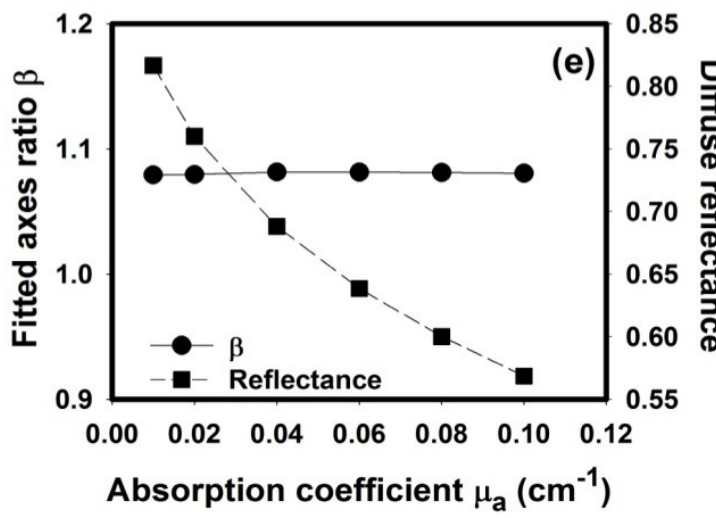

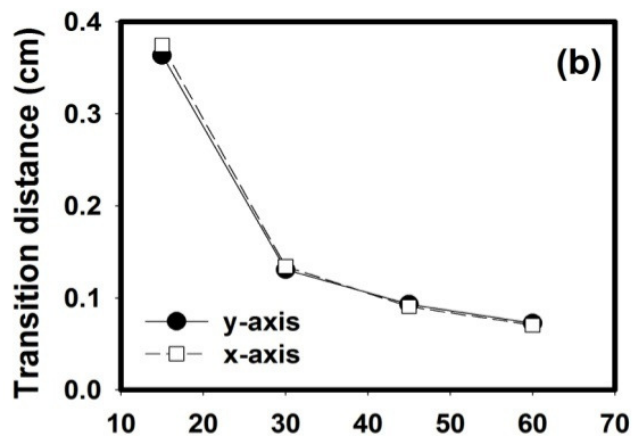

Background scattering $\mu_{\mathbf{s}, \mathbf{b}}\left(\mathrm{cm}^{-1}\right)$
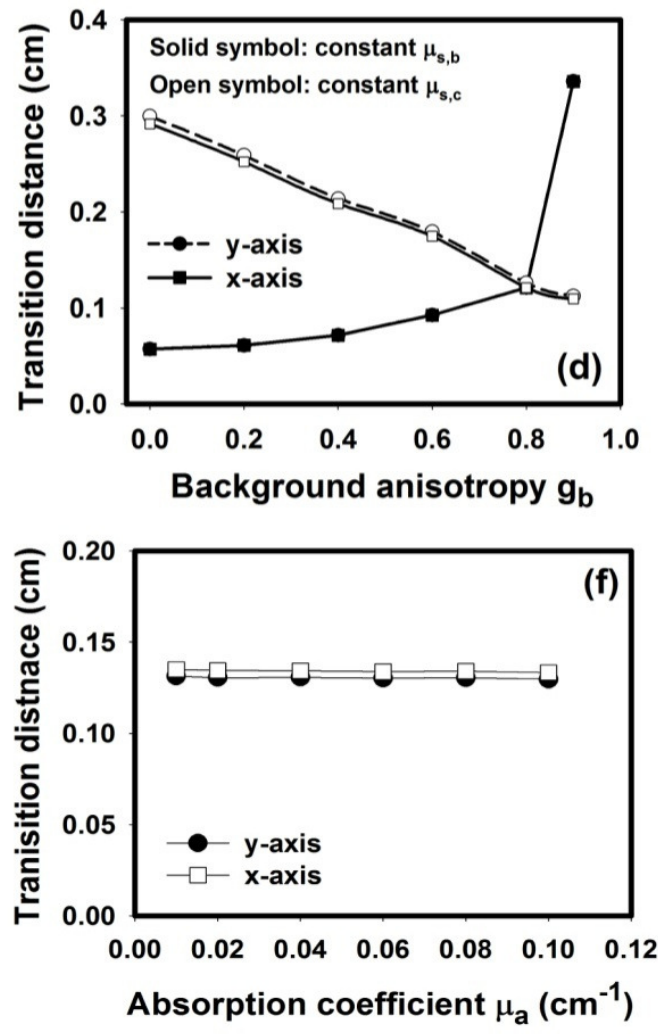

Figure 2-5: The fitted axes ratio $\beta$ and total diffuse reflectance at different (a) background scattering coefficient $\mu_{\mathrm{s}, \mathrm{b}}$, (c) background anisotropy $g_{\mathrm{b}}$, and (e) absorption coefficient $\mu_{\mathrm{a}}$. The corresponding transition distances were shown in (b), (d), and (f), respectively. Unless otherwise indicated, the optical properties in the simulation were $r=0.1 \mu \mathrm{m} ; \mu_{\mathrm{s}, \mathrm{b}}=30 \mathrm{~cm}^{-1} ; g_{\mathrm{b}}=0.8 ; \mu_{\mathrm{a}}=$ $0.01 \mathrm{~cm}^{-1}$ and $\sqrt{\mu_{s}^{\prime}(x) / \mu_{s}^{\prime}(y)}=1.08$.

Figure 2-5 shows the effects of background optical properties on the geometric profiles of the simulated diffuse reflectance. We also calculated the transition distance as defined earlier and the total diffuse reflectance. Unless otherwise indicated, the optical 
properties used in the simulation were: the cylinder radius of $r=0.1 \mu \mathrm{m}, n_{c}=1.46, \mu_{s, b}=$ $30 \mathrm{~cm}^{-1}, g_{\mathrm{b}}=0.8, \mu_{a}=0.01 \mathrm{~cm}^{-1} \cdot \sqrt{\mu_{s}^{\prime}(x) / \mu_{s}^{\prime}(y)}$ was maintained at a constant value of 1.08 .

Figure 2-5(a) shows the fitted axes ratio $\beta$ and total diffuse reflectance at different background scattering coefficients from $\mu_{s, b}=15$ to $60 \mathrm{~cm}^{-1}$. In order to maintain $\sqrt{\mu_{s}^{\prime}(x) / \mu_{s}^{\prime}(y)}$, the cylinder volume densities were varied from $\rho=0.07 \%$ to $0.27 \%$, respectively. The result indicated that the background scattering coefficient had no significant effect on the fitted axes ratio when $\sqrt{\mu_{s}^{\prime}(x) / \mu_{s}^{\prime}(y)}$ was the same. As expected, the total diffuse reflectance increased with the background scattering coefficient. However, the transition distance decreased as the background scattering coefficient increased as shown in Fig. 2.5(b). The transitional distances calculated along the cylinder were nearly identical to those calculated perpendicular to cylinders.

Figure 2-5(c) shows the fitted axes ratio $\beta$ and total diffuse reflectance at different background anisotropy values. When increasing the background $g_{\mathrm{b}}$ from 0.0 to 0.9 , the $\sqrt{\mu_{s}^{\prime}(x) / \mu_{s}^{\prime}(y)}$ was maintained as a constant of 1.08 by either (1) maintaining background scattering $\mu_{\mathrm{s}, \mathrm{b}}$ and reducing cylinder volume density $\rho$ from $0.69 \%$ to $0.07 \%$ or (2) maintaining cylinder volume density $\rho$ and increasing background scattering coefficient $\mu_{\mathrm{s}, \mathrm{b}}$ from 6.0 to $60.0 \mathrm{~cm}^{-1}$. In either cases, the background anisotropy $g_{\mathrm{b}}$ had no impact on the fitted axes ratio $\beta$. The total diffuse reflectance decreased as the background anisotropy increased when reducing cylinder volume concentration (and thus the cylinder scattering coefficient $\mu_{s, c}$ ). But it did not change with $g_{\mathrm{b}}$ when increasing $\mu_{\mathrm{s}, \mathrm{b}}$ but maintaining the $\mu_{s, b}^{\prime}$. On the other hand, the transition distance decreased with the 
background anisotropy when increasing the $\mu_{s, b}$; whereas the transition distance increased with the background anisotropy when reducing $\mu_{s, c}$ as shown in Fig. 2.5(d).

Figure 2-5(e) shows the fitted axes ratio $\beta$ and total diffuse reflectance at different background absorption coefficients from $\mu_{\mathrm{a}}=0.01$ to $0.1 \mathrm{~cm}^{-1}$. The cylinder volume density was kept at $\rho=0.14 \%$. The result indicated that the total diffuse reflectance decreased as the absorption coefficient increased. However, background absorption coefficient had no significant effect on the axes ratio and the transition distance as shown in Fig. 2-5(f).

\subsection{Discussion}

As predicted from the ADE and CTRW theories, Monte Carlo simulation showed that the equi-intensity reflectance in a fibrous turbid medium had an elliptic shape. The elliptical patterns were inclined to the fiber axis where the total reduced scattering coefficient $\mu_{\mathrm{s}}$ ' is the smallest than in any other direction. The ADE also predicts that the ratio of the two orthogonal axial lengths in the elliptical distribution is inversely proportional to the square root of corresponding reduced scattering coefficients perpendicular and parallel to the fibrous structures (Dagdug et al. 2003; Heino et al. 2003): $r_{y} / r_{x}=\sqrt{\mu_{s}^{\prime}(x) / \mu_{s}^{\prime}(y)}$. However, the Monte Carlo simulation indicated that this is true only for small cylinders. At a constant $\sqrt{\mu_{s}^{\prime}(x) / \mu_{s}^{\prime}(y)}$, the elliptical equi-intensity patterns were much less elongated for cylinders of $1.5 \mu \mathrm{m}$ radius than those of $0.1 \mu \mathrm{m}$ radius. 
The observed size dependency is likely due to the different scattering efficiency and anisotropy profiles at different cylinder sizes. Figure 2-6 shows the scattering efficiency and anisotropy calculated for cylinders of different radii. Both the scattering efficiency and anisotropy depended on the incident direction of the photon relative to the cylinder axis. The anisotropy of small cylinders acted more like an isotropic than anisotropic scatterer at large incident angles of $\xi>45^{\circ}$ because of the small anisotropy. On the contrary, for cylinders of a large radius, the anisotropy was $\sim 0.9$ at large incident angles, indicating a predominantly forward scattering. Therefore, photons that were incident at larger angles had much less chance to be scattered back toward the large cylinders than small cylinders. In addition, when $\xi>30^{\circ}$ the scattering efficiency value for large cylinders was significantly larger than for the small cylinders. So photons experienced more scattering by large cylinders at large incident angles. Overall, photons propagated farther along the cylinders than across the cylinders, but such inclination was greater for small cylinders. The fitted axes ratio $\beta$ in anisotropic media of small cylinders was closer to the ADE prediction which appeared to be an extreme limit for small cylinders. 

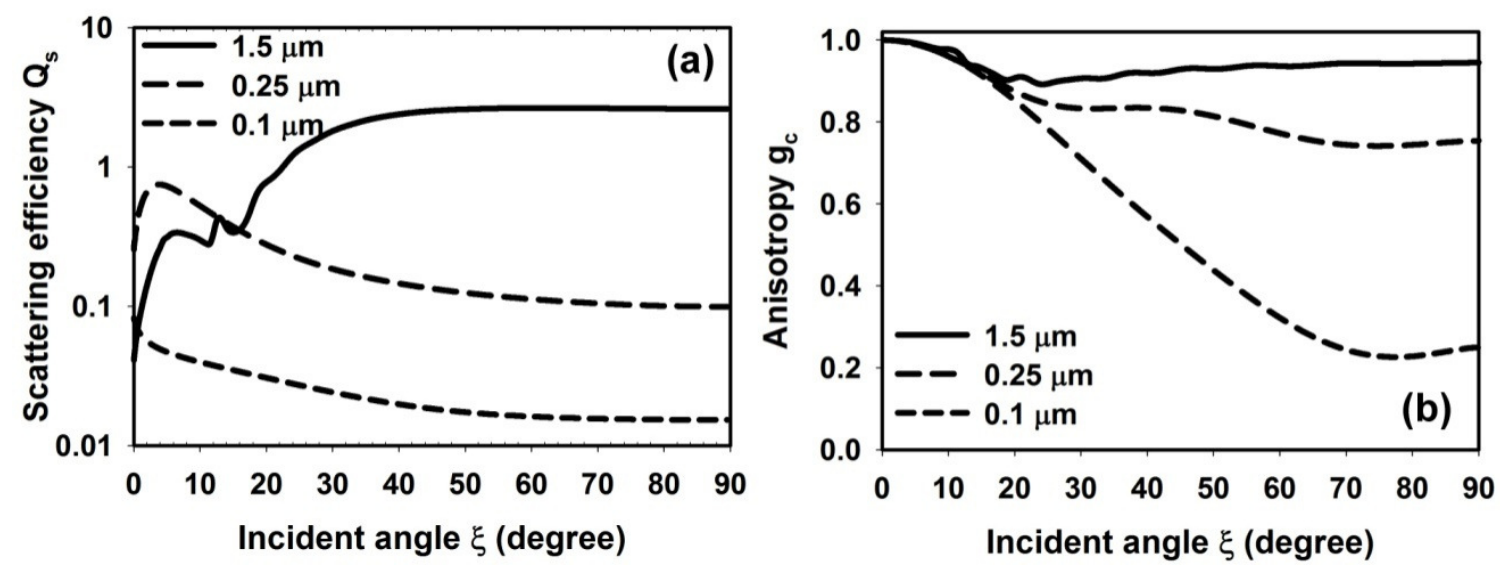

Figure 2-6: (a) The scattering efficiency $\mathrm{Q}_{\mathrm{s}}$ and (b) anisotropy $\mathrm{g}_{\mathrm{c}}$ as a function of incident angle $\xi$ for cylinders of different radii.

We found that when $\sqrt{\mu_{s}^{\prime}(x) / \mu_{s}^{\prime}(y)}$ was maintained at a constant value, the fitted axes ratio $\beta$ was not affected by varying the background optical properties when evaluated far away from the incident point. In other words, the geometric shape of the diffuse reflectance was only determined by the ratio of the total reduced scattering coefficient along the two primary axes. However, the total diffuse reflectance was affected by all of the background optical properties. In agreement with diffuse theory, the total diffuse reflectance generally decreased as the absorption coefficient increased, and increased as reduced scattering coefficient increased.

The transition distance was also affected by the scattering coefficient. It is known that single scattering is important at locations closer to the incident point, while multiple scattering becomes dominant at larger distances. At a certain distance from the incident, a photon undergoes a higher number of scattering events in samples with higher scattering coefficients. In other words, a higher background scattering coefficient reduces the transport distance required for the transition from single scattering to multiple scattering, 
which results in a smaller transition distance as shown in Figure 2-5(b). The effects of background anisotropy (Figure 2-5(d)) can also be explained in the same way due to the changes in either the background scattering coefficient or the cylinder scattering coefficient. Because the overall absorption coefficients were much smaller than the scattering coefficients, changes in absorption coefficient had little effect on the transition distance.

\subsection{Conclusion}

In summary, Monte Carlo simulation was employed to simulate the propagation of light in fibrous anisotropic scattering medium. The spatial profiles of the diffuse reflectance were quantitatively analyzed using a numerical fitting algorithm. The results indicated that the equi-intensity profiles of the surface reflectance always had elliptic distributions when evaluated at larger distances from the incidence. The shape of the elliptical distribution was not affected by the background optical properties when the ratio of the total reduced scattering coefficients parallel and perpendicular to the cylinder was maintained constant. The prediction of the anisotropic diffuse equation theory was in agreement with the Monte Carlo simulation only for small cylinders. As fiber diameters in biological tissue may vary from tens of nanometers to hundreds of microns, caution should be taken when applying diffuse theory to study light propagation in tissues. 


\section{CHAPTER 3}

\section{DETERMINING THE OPTICAL PROPERTIES IN A FIBROUS TURBID MEDIUM USING TIME-RESOLVED REFLECTANCE ${ }^{2}$}

\subsection{Introduction}

The optical properties of biological tissue affect the light propagation. For instance, the size and concentration of scatters in tissue affect how photons are scattered. Knowledge of such interactions is essential for developing optical techniques to derive tissue structure properties, such as size, concentration, and alignment of fibers which strongly influence the tissue mechanical properties (Lake et al. 2009; Morgan et al. 2006; Parry and Craig 1984). In many optical studies, tissue samples are often assumed to be isotropic, i.e., the optical properties are independent of the measurement direction. However, this assumption is invalid for many biological tissue such as muscle (Marquez et al. 1998), skin (Nickell et al. 2000), and dentin (Kienle et al. 2003) because they contain fibrous and tubular structures that are anisotropic. For instance, Marquez et al. (1998) used the spatial resolved technique and found the optical properties on chicken breast were different depending on the measurement direction to the fiber.

\footnotetext{
2 Materials in this Chapter were published in Shuaib, A. and G. Yao "Determining the optical properties in a fibrous turbid medium,” Proc. SPIE 7897, 78978Q (2011). doi:10.1117/12.873007.
} 
Several models, such as anisotropic diffuse equation (ADE) (Heino et al. 2003; Johnson et al. 2002) and continues time random walk (CTRW) (Dagdug et al. 2003), predicted that the equi-intensity profile of the surface reflectance has an elliptical shape in medium that consists of cylindrical shape scatters (such as aligned fibers). This is confirmed experimentally in several studied with various fibrous tissue models (Hebden et al. 2004; Ranasinghesagara et al. 2006; Ranasinghesagara and Yao 2007). Previously, ADE was shown to be capable of predicating the elliptical shape in samples of small fibers (Shuaib and Yao 2010). Kienle et al. (2004) developed a Monte Carlo method to simulate light propagation in a tissue model that consists of aligned infinite long cylinders and spherical scatters to explain the pattern of spatially resolved reflectance in dentin and arteries. Kienle showed (2007) that the ADE model did not correctly predict the spatially resolved reflectance in fibrous turbid media. In addition, the derived reduced scattering coefficient using the time-resolved isotropic model (Kienle et al. 2007) varied with the direction of the fibers. The derived reduced scattering coefficient parallel to the fiber direction was close to the background reduced scattering coefficient; whereas the derived reduced scattering coefficient measured in a perpendicular direction was different and larger than any other direction. The difference between perpendicular and parallel measured reduced scattering coefficients was found to increase when the ratio of cylinder and background reduced scattering coefficient increases. On the other hand, the derived absorption coefficient is independent of the direction of the fibers, although the distance between the incident light and the detector needs to be large $\left(\rho>>1 / \mu_{s}^{\prime}\right)$ to obtain the correct value (Kienle et al. 2007). 
In this paper, we further investigate the possibility of retrieving separately the scattering coefficients of the cylindrical component and the background isotropic component in fibrous tissues by using the time-resolved reflectance. We implemented the Monte Carlo method (Kienle et al. 2004) to simulate surface reflectance in a fibrous tissue that contains both background isotropic scatters and infinite long cylinders. Isotropic diffuse equation was then used to derive the optical properties of the Monte Carlo generated reflectance data. The optical coefficients derived from different measurement directions were studied. The derived reduced scattering coefficient, when measured along the cylindrical fibers, had good agreement with the true background scattering coefficients. When measured perpendicularly to the cylinders the derived reduced scattering coefficient was close to the summation of the reduced scattering coefficients of both cylinders and background in samples with small cylinders. If the fiber size in the medium is known, the reduced scattering coefficient associated with the cylinders can be derived by using a correction coefficient.

\subsection{Method}

\subsubsection{Monte Carlo simulation}

A pencil beam $(\lambda=800 \mathrm{~nm})$ was incident on the semi-infinite fibrous medium as shown in Fig. 3-1. The Monte Carlo simulation process is described in detail previously (Kienle et al. 2004; Shuaib and Yao 2010). The semi-infinite fibrous medium contains aligned cylinder structures that are parallel to the surface. The sample is assumed to have a single homogenous isotropic absorption coefficient $\mu_{a}$. The spherical particles were 
assumed to have isotopic optical properties (i.e. independent of the incident direction) characterized by the background scattering coefficient $\mu_{s, b}$ and background anisotropy $g_{b}$ using a Henyey-Greenstein phase function. The scattering coefficient of the cylinder $\mu_{s, c}$ at angle $\xi$ was calculated as the following:

$$
\mu_{s, c}(\xi)=2 \mathrm{r} * \mathrm{c}_{\mathrm{A}} * \mathrm{Q}_{\mathrm{s}}(\xi)
$$

where $r$ is the cylinder radius, $\mathrm{c}_{\mathrm{A}}$ is the concentration of cylinders $\left(\mathrm{mm}^{-2}\right)$, and $\mathrm{Q}_{\mathrm{s}}(\xi)$ is the scattering efficiency of a single cylinder calculated using Yousif and Boutros's method (Yousif and Boutros 1992). Angle $\xi$ is defined as the angle between the direction of the incident photon and that of the cylinder axis. When the incident photon is parallel to the cylinder axis the angle $\xi=0^{\circ}$; whereas if the incident photon is perpendicular to the cylinder axis the angle $\xi=90^{\circ}$.

The scattered light by the cylinder is restricted to a cone with the cylinder as the axis of the cone has a half angle $\xi$. The scattering phase function can be calculated using the algorithm by Yousif and Boutros (1992). When the incident light is parallel to the cylinder axis $\left(\xi=0^{\circ}\right)$, the cylinder anisotropic factor $g_{c}=1$; therefore, the light is not deflected and the cylinder reduced scattering coefficient $\mu_{s, c}^{\prime}\left(0^{\circ}\right)=0$. The total reduced scattering in the sample was defined as $\mu_{s, t}^{\prime}=\mu_{s}^{\prime}\left(90^{\circ}\right)=\mu_{s, c}^{\prime}\left(90^{\circ}\right)+\mu_{s, b}^{\prime}$.

The Monte Carlo simulation has a temporal resolution of $5.0 \mathrm{ps}$ and a spatial resolution of the $0.05 \mathrm{~cm}$. Reflectance measurement positions parallel $\left(0^{\circ}\right)$ and perpendicular $\left(90^{\circ}\right)$ to cylinder structure direction at distance away from the incident light $d=0.95 \mathrm{~cm}$ were obtained from the Monte Carlo simulation. 


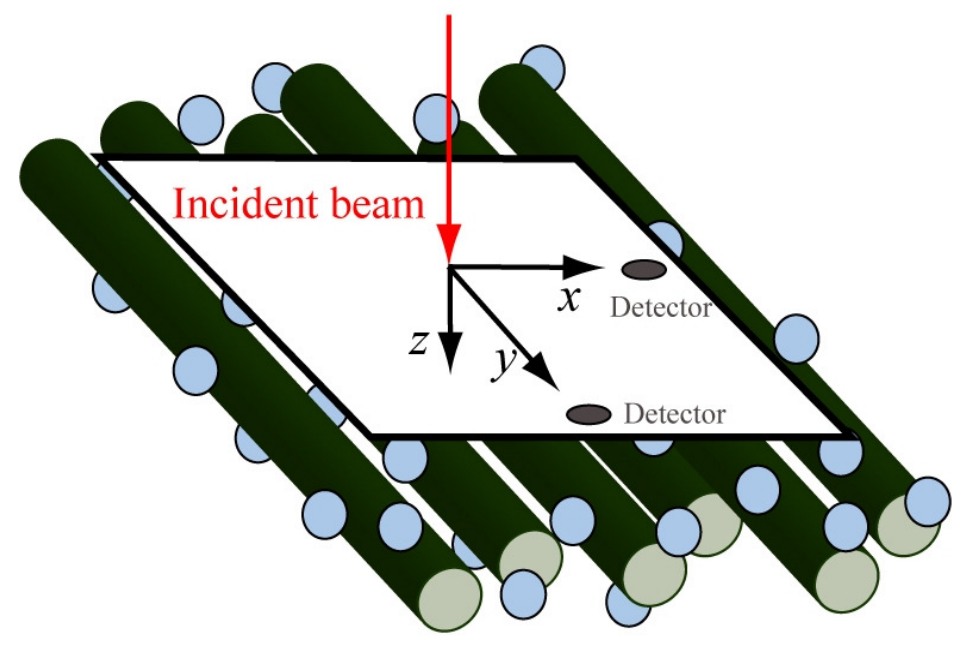

Figure 3-1: The schematic diagram of the sample model used in the Monte Carlo

\subsubsection{Diffuse theory model}

For the determination of the optical properties of the fibrous turbid medium, the solution of the isotropic diffusion theory is used to obtain the optical properties from the time-resolved measurement (Kienle and Patterson 1997; Kienle et al. 2007). An extrapolated boundary condition (EBC) isotropic diffuse equation for time-resolved measurement is as follows:

$$
R(d, l)=\frac{c e^{-\mu_{a} l} e^{-\frac{\rho^{2}}{4 D l}}}{2(4 \pi D)^{3 / 2}(l)^{5 / 2}}\left[z_{o} e^{\frac{-z_{o}^{2}}{4 D l}}+\left(z_{o}+2 z_{b}\right) e^{\frac{-\left(z_{o}+2 z_{b}\right)^{2}}{4 D l}}\right]
$$

where $D=1 /\left(3 \cdot\left(\mu_{s}^{\prime}+\mu_{a}\right)\right)$ is the diffusion constant, $c$ is the speed of light in turbid medium, $\rho$ is the measurement distance from the incident location, $\mu_{s}$ is the scattering coefficient, $g$ is the anisotropic factor, and $\mu_{s}^{\prime}$ is the reduced scattering coefficient and is 
$(1-g) \mu_{s} ; z_{0}=1 / \mu_{s}$ is the transport mean free path; $z_{b}=2 A D, A=\left(1+R_{\text {eff }}\right) /\left(1-R_{\text {eff }}\right)$; where $R_{\text {eff }}$ is the fraction of photon that are internally diffusely reflected at the boundary (Haskell et al. 1994). A Levenberg-Marquadt nonlinear least squares fitting algorithm in Matlab Curve Fitting Toolbox 3.0 (The Mathworks Inc., Natick, MA) was used to retrieve the optical properties. The range of the data points used in fitting program was $85 \%$ and $0.1 \%$ of the maximum value for the leading and tailing portion, respectively.

For convenience, the derived reduced scattering and absorption coefficients when the measurement is parallel $\left(0^{\circ}\right)$ and perpendicular $\left(90^{\circ}\right)$ to the cylinder direction are defined as $\mu_{s, 0}^{\prime}, \mu_{a, 0}$ and $\mu_{s, 90}^{\prime}, \mu_{a, 90}$, respectively.

\subsection{Results}

Figure 3-2(a) shows an example of the spatial resolved reflectance image of anisotropic medium where the cylinder structures were aligned along the $y$-axis. The cylinders had a radius of $r=0.25 \mu \mathrm{m}$, a refractive index of $n_{c}=1.46$, a volume density of $\rho=0.8 \%$, and a background refractive index $n_{b}=1.36$. When the incident light is perpendicular to the cylinder $\left(\xi=90^{\circ}\right)$, the corresponding scattering coefficient was $\mu_{s, c}=$ $20.32 \mathrm{~cm}^{-1}$ and $g_{c}=0.754$. The background spherical scatters had $\mu_{s, b}=40 \mathrm{~cm}^{-1}$ and $g_{b}=$ 0.8 . The absorption coefficient was $\mu_{a}=0.1 \mathrm{~cm}^{-1}$. It can be seen that the equi-intensity has an elliptical shape orientated perpendicular to the fiber direction at close distance from the incident light. As the distance away from the incident light increase, the equi-intensity shape goes through a transition where it changes continuously. Then, at a large distance from the incident light, the equi-intensity stabilizes and its elliptical shape direction becomes parallel to the fiber direction. The time-resolved measurements parallel $\left(0^{\circ}\right)$ and 
perpendicular $\left(90^{\circ}\right)$ to the fiber direction at $\rho=0.95 \mathrm{~cm}$, and the results of the isotropic diffuse fit are shown in Fig. 3-2(b). The diffuse equation can fit the simulation results very well.
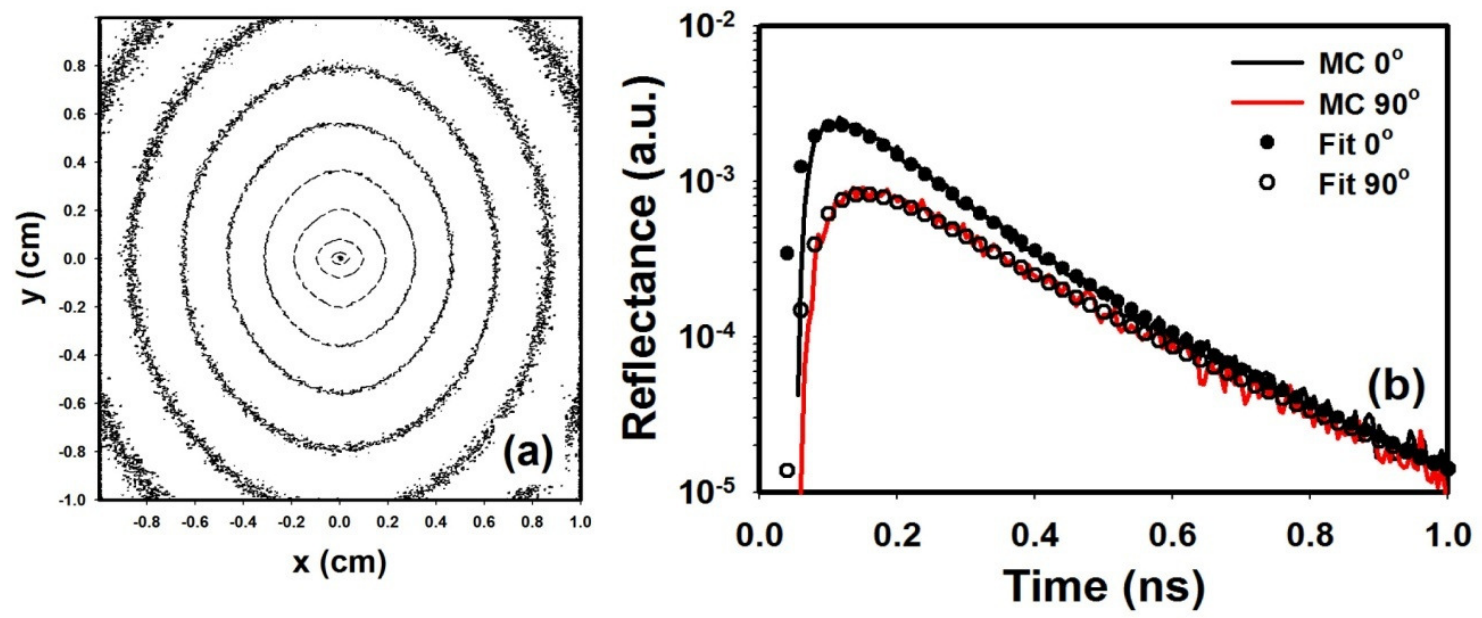

Figure 3-2: (a) Sample spatial resolved reflectance image obtained in anisotropic medium. The cylinders were aligned along the y-axis. The image size is $1 \times 1 \mathrm{~cm}^{2}$. (b) The time-resolved measurements parallel $\left(0^{\circ}\right)$ and perpendicular $\left(90^{\circ}\right)$ to the fiber direction (solid lines) at $\rho=0.95$ $\mathrm{cm}$ and their isotropic diffuse model (circles).

Figure 3-3(a-b) shows the derived reduced scattering coefficient parallel $\left(\mu_{s, 0}^{\prime}\right)$ and perpendicular $\left(\mu_{s, 90}^{\prime}\right)$ to the cylinder direction for different cylinder radii with different total reduced scattering coefficient $\left(\mu_{s, t}^{\prime}\right)$. The cylinder reduced scattering coefficient $\left(\mu_{s, c}^{\prime}\right)$ ranged from 2 to $10 \mathrm{~cm}^{-1}$ and the background reduced scattering coefficient $\left(\mu_{s, b}^{\prime}\right)$ was maintained at a constant value of $8 \mathrm{~cm}^{-1}$ (dotted line in Fig. 3-3a). For all different cylinder sizes, the derived $\mu_{s, 0}^{\prime}\left(\approx 8 \mathrm{~cm}^{-1}\right)$ was close to the true background scattering coefficient $\left(\mu_{s, b}^{\prime}\right)$ with an error less than $10 \%$. However, the derived $\mu_{s, 90}^{\prime}$ showed a strong dependency on the cylinder size. The derived $\mu_{s, 90}^{\prime}$ increased linearly with total reduced scattering coefficient $\left(\mathrm{R}^{2} \geq 0.99\right.$ in Fig. $\left.3-3 b\right)$. The different sizes of cylinder of $0.1,0.25$, and $1.5 \mu \mathrm{m}$ had a linear slope of: $0.90,0.81$, and 0.24 , respectively. Only when the 
cylinder size is small, the derived $\mu_{s, 90}^{\prime}$ was close to the true value of the total reduced scattering coefficient $\left(\mu_{s, t}^{\prime}\right)$. The linear slopes in Figure 3-3(b) are similar with fitted axes ratio of the spatial-resolved measurements with different cylinder sizes in our previous study (Shuaib and Yao 2010) (Fig. 2-3).
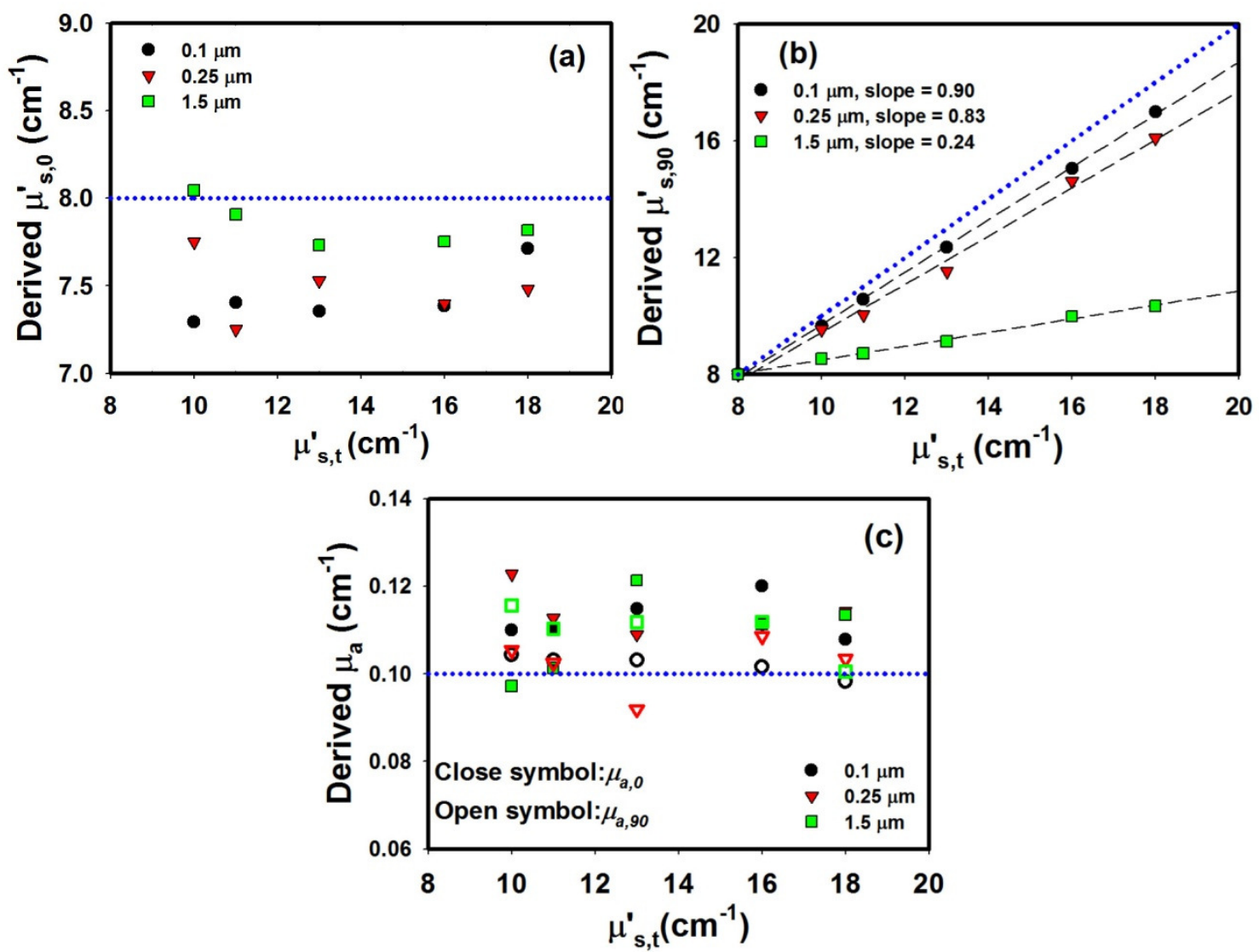

Figure 3-3: The derived reduced scattering coefficients (a) parallel $\mu_{\mathrm{s}, 0}^{\prime}$ and (b) perpendicular $\mu_{\mathrm{s}, 90}^{\prime}$ to the fiber direction as a function of the total reduced scattering coefficient, $\mu_{s, t}^{\prime}$. (c) The derived absorption coefficients parallel $\mu_{a, 0}$ and perpendicular $\mu_{a, 90}$ to the fiber direction as a function of actual total reduced scattering coefficient, $\mu_{s, t}^{\prime}$. The optical properties used in the simulation were $\mu_{s, c}^{\prime}=2,3,5,8$ and $10 \mathrm{~cm}-1, \mu_{s, b}^{\prime}=8 \mathrm{~cm}^{-1}, g_{b}=0.8$, and $\mu_{a}=0.1 \mathrm{~cm}^{-1}$. The dotted lines indicate the theoretical values.

Figure 3-3(c) shows the derived absorption coefficient of both parallel $\mu_{a, 0}$ and perpendicular $\mu_{a, 90}$ for different cylinder radii with different total scattering coefficient $\left(\mu_{s, t}^{\prime}\right)$. It shows that the absorption coefficient is independent of the measurement direction and the average absorption coefficient error is less than $22 \%$. Often, the derived 
absorption coefficient when the measurement is parallel to the fiber direction is larger than that measured perpendicularly. Part of this is due to the requirement that the distance between the detector and the incidence needs to be larger when the measurement is parallel $\left(\rho>>1 / \mu_{s}^{\prime}\right)$ to obtain the true absorption coefficient. Although the noise is fairly large in the measurement, it can be reduced by increasing the number of simulated photons.

To validate the derived $\mu_{s, 0}^{\prime}$ is equal to the background reduced scattering coefficient, the effect of background optical properties was investigated as shown in Figure 3-4. The optical properties used in the simulation were $r=0.1 \mu \mathrm{m}, \mu_{s, b}^{\prime}=40 \mathrm{~cm}^{-1}$, $g_{b}=0.8, \mu_{a}=0.1 \mathrm{~cm}^{-1}$, and $\sqrt{\mu_{s}^{\prime}(x) / \mu_{s}^{\prime}(y)}=1.275$.

Figure 3-4(a) shows the derived $\mu_{s, 0}^{\prime}$ and $\mu_{s, 90}^{\prime}$ at different background scattering coefficient from $\mu_{s, b}^{\prime}=20$ to $120 \mathrm{~cm}^{-1}$. The $\mu_{s, 0}^{\prime}$ and $\mu_{s, 90}^{\prime}$ were both close to the true value. To be noted, the $\mu_{s, c}^{\prime}$ is very close to the true value due to the cylinders' small size. Figure 3-4(b) shows the derived $\mu_{a, 0}$ and $\mu_{a, 90}$ at different background scattering coefficient. The derived absorption coefficient is close to the true $\mu_{a}$ for large background reduced scattering coefficient $\mu_{s, b}^{\prime}$ value; however, as the $\mu_{s, b}^{\prime}$ value decreases the derived absorption coefficient error becomes large. This is because the distance between the incident light and the detector $\left(\rho>>1 / \mu_{s}^{\prime}\right)$ must be large to obtain the correct absorption coefficient $\mu_{a}$ value. Figure 3-4(c) shows the derived $\mu_{s, 0}^{\prime}$ and $\mu_{s, 90}^{\prime}$ at different background anisotropy from $g_{b}=0.0$ to 0.9 . The background anisotropic factor has no significant effect on both derived reduced scattering coefficients $\left(\mu_{s, 0}^{\prime}\right.$ and $\left.\mu_{s, 90}^{\prime}\right)$ and are close to the true value. Figure 3-4(d) shows the derived $\mu_{a, 0}$ and $\mu_{a, 90}$ at a different background anisotropic factor $g_{b}$. The derived $\mu_{s, 0}^{\prime}$ is close to the true value and not 
affected by altering the background reduced scattering coefficient. Figure 3-4(e) shows the derived $\mu_{s, 0}^{\prime}$ and $\mu_{s, 90}^{\prime}$ at different absorption coefficient values from $\mu_{a}=0.05$ to 0.3 $\mathrm{cm}^{-1}$. The absorption coefficient had no significant effect on the derived reduced scattering coefficients. Figure 3-4(f) shows the derived $\mu_{a, 0}$ and $\mu_{a, 90}$ at different absorption coefficient values. The derived absorption coefficient $\mu_{a, 90}$ is close to the true value and not significantly affected by altering the background reduced scattering coefficient. The derived $\mu_{a, 0}$ is accurate for small absorption coefficient values. However, as the absorption coefficient increases, the derived $\mu_{a, 0}$ error increases as well. This is due to fewer photons reaching the detector. In other words, the signal-to-noise is dramatically decreased. A significant increase in the number of photons is needed to obtain high accuracy in retrieved absorption coefficient $\mu_{a}$ value. 

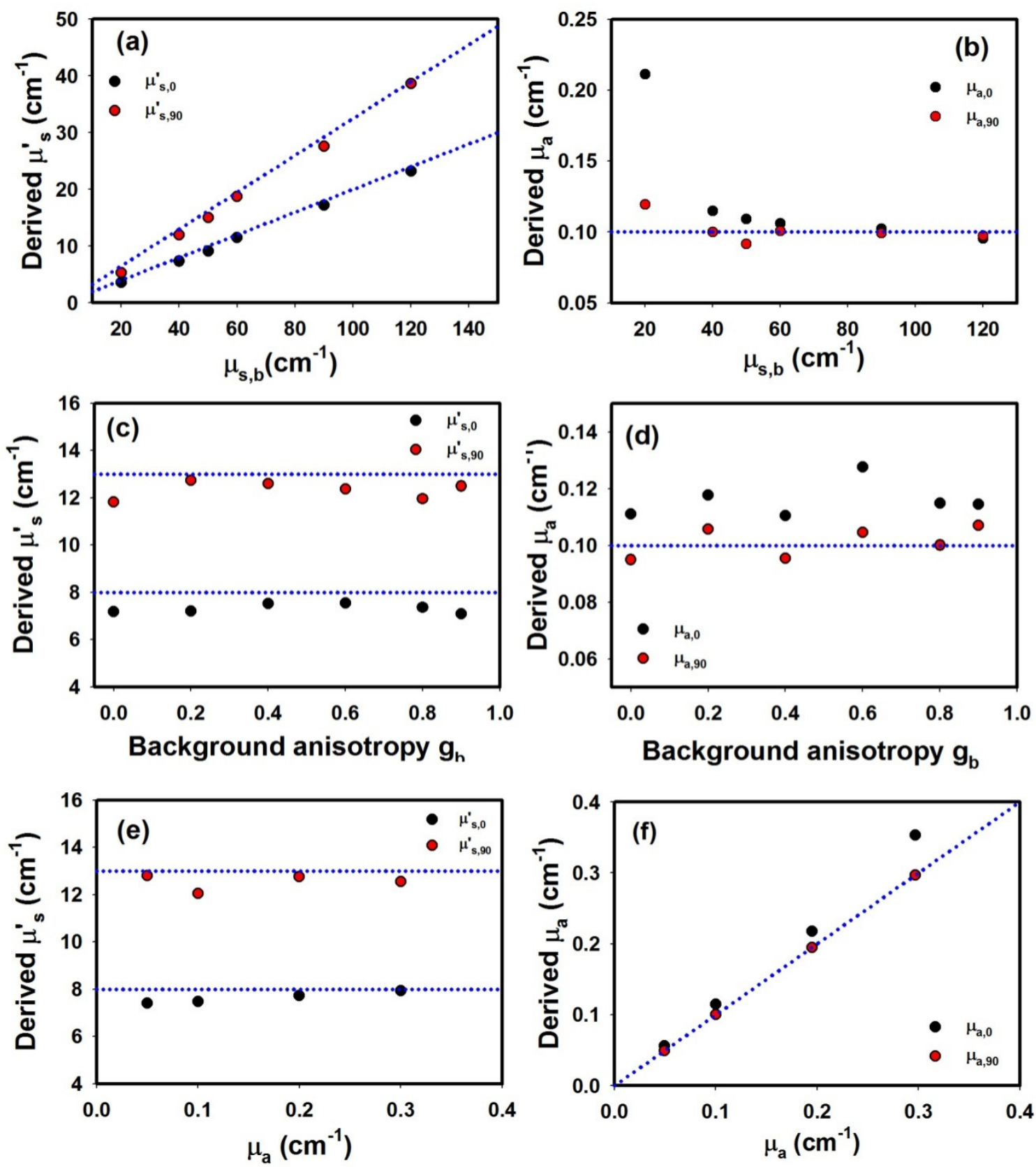

Figure 3-4: The derived reduced scattering coefficients parallel $\mu_{s, 0}^{\prime}$ and perpendicular $\mu_{s, 90}^{\prime}$ to the fiber direction at different (a) background scattering coefficients $\mu_{s, b}$, (c) background anisotropies $g_{b}$, and (e) absorption coefficients $\mu_{a}$. The corresponding average derived absorption coefficients are shown in (b), (d), and (f), respectively. Unless otherwise indicated, the optical properties in the simulation were $r=0.1 \mu \mathrm{m}, \mu_{s, b}^{\prime}=8 \mathrm{~cm}^{-1}, g_{b}=0.8, \mu_{a}=0.1 \mathrm{~cm}^{-1}$, and $\sqrt{\mu_{s}^{\prime}(x) / \mu_{s}^{\prime}(y)}=1.275$. 


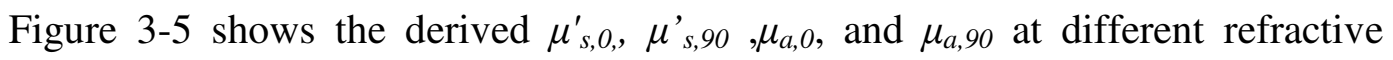
index, $n_{c}$, from 1.44 to 1.48 . It can be seen that the small variation in cylinder refractive index $n_{c}$ had an insignificant effect on the determined optical properties.
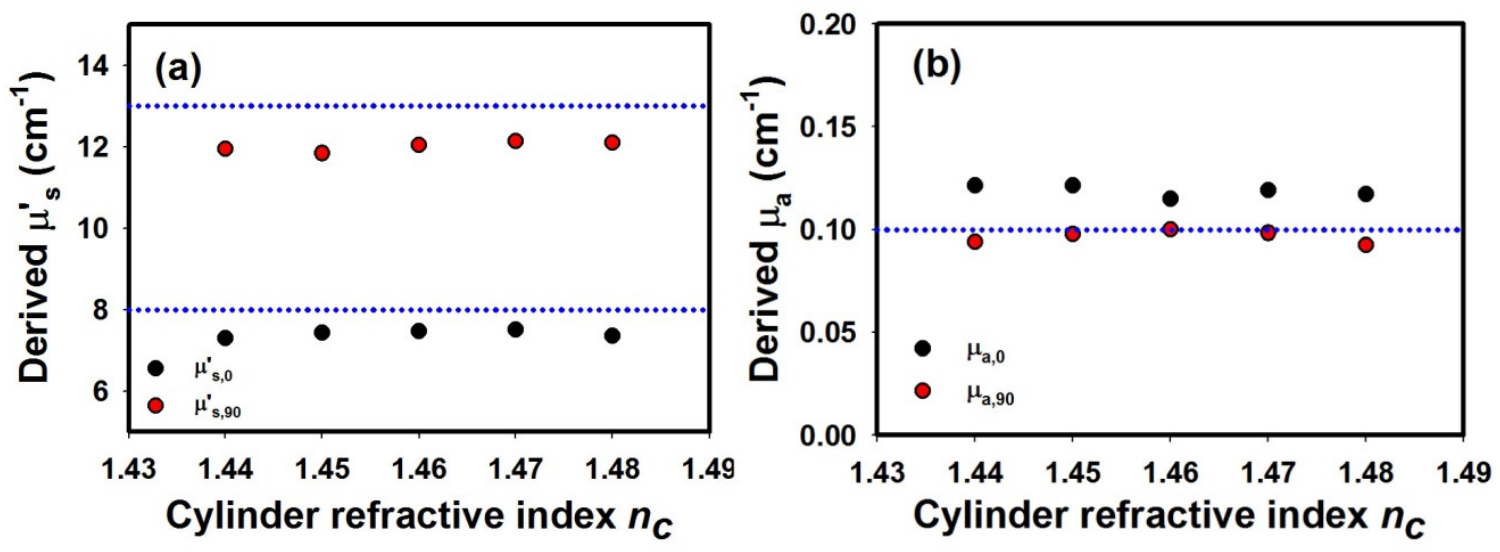

Figure 3-5: The derived (a) reduced scattering coefficient parallel $\mu_{s, 0}^{\prime}$ and perpendicular $\mu_{s, 90}^{\prime}$ and (b) absorption coefficient parallel $\mu_{a, 0}$ and perpendicular $\mu_{a, 90}$ to the fiber direction at different cylinder refractive index. Unless otherwise indicated, the optical properties in the simulation were $r=0.1 \mu \mathrm{m}, \mu_{s, c}^{\prime}=13 \mathrm{~cm}^{-1} ; \mu_{s, b}^{\prime}=8 \mathrm{~cm}^{-1}, g_{b}=0.8, \mu_{a}=0.1 \mathrm{~cm}^{-1}$, and $\sqrt{\mu_{s}^{\prime}(x) / \mu_{s}^{\prime}(y)}=1.275$.

Based on the above results, knowledge of the fiber size is needed to obtain the true reduced scattering coefficient. The reduced scattering coefficient associated with cylinder can be derived with the following equation:

$$
\begin{gathered}
\mu_{s, 0}^{\prime}=\mu_{s, b}^{\prime} ; \mu_{s, 90}^{\prime}=\mu_{s, b}^{\prime}+\mu_{s, c}^{\prime}\left(90^{o}\right) \\
\text { Corrected } \mu_{s, c}^{\prime}\left(90^{o}\right)=\frac{\mu_{s, 90}^{\prime}-\mu_{s, 0}^{\prime}}{C(r)}=\frac{\mu_{s, c}^{\prime}\left(90^{o}\right)}{C(r)}
\end{gathered}
$$

where $\mathrm{C}(r)$ is a fiber-size dependent factor correction coefficient that is obtained from the slope of each cylinder radii in Figure 2-3. Figure 3-6 shows the corrected derived cylinder reduced scattering coefficient $\left(\mu_{s, c}^{\prime}\right)$. It can be clearly seen that the $\mu_{s, c}^{\prime}$ is close to the true value. The error of the corrected derived $\mu_{s, c}^{\prime}$ is less than $10 \%$. 


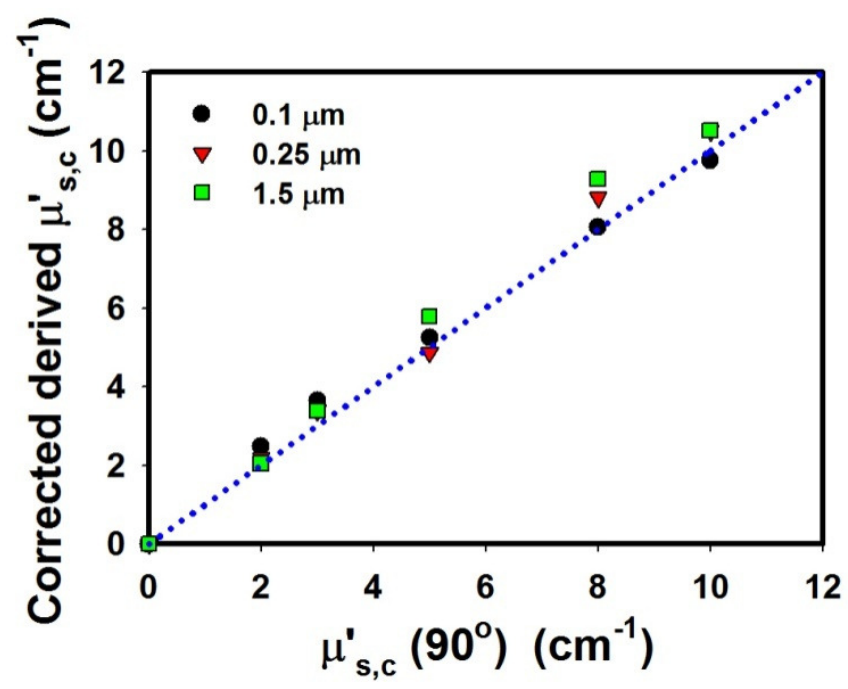

Figure 3-6: The corrected reduced scattering coefficient $\mu_{s, c}^{\prime}$ obtained by using the Eq. (3-3) compared with the true cylinder reduced scattering coefficient $\mu_{s, c}^{\prime}\left(90^{\circ}\right)$.

\subsection{Discussion and Conclusion}

A Monte Carlo simulation was used to simulate the light propagation in fibrous tissue that contains aligned cylinders and spherical shape scatters. An isotropic diffuse model was applied to retrieve the optical properties in anisotropic tissue with different fiber sizes from simulated time-resolved reflectance. The derived reduced scattering coefficient for measurement along the fiber direction was in good agreement with the true background reduced scattering coefficient. On the other hand, the derived reduced scattering coefficients for measurement perpendicular to the fiber direction was close the total reduced scattering coefficient for medium with small cylinder radii. The results show that the isotropic diffuse model is incapable of retrieving the true $\mu_{s}^{\prime}$. In other words, isotropic diffuse model does not describe correctly the light propagation in the fibrous tissue unless the cylinder diameter is small. Knowing the fiber size in the medium is necessary to obtain the true cylinder reduced scattering coefficient. 
The ADE and CTRW theories predicted that the equi-intensity distribution of the surface reflectance in fibrous turbid medium has an elliptical shape. The elongation of the ellipse is along the fiber direction where the reduced scattering coefficient is the smallest. Moreover, the ADE predicted that the axes ratio of the elliptical shape is equal to the inverse of the square root of the corresponding reduced scattering coefficient parallel and perpendicular to the fiber direction (Dagdug et al. 2003; Heino et al. 2003). The axis ratio can be calculated using the method describe in previous studies (Li et al. 2008; Ranasinghesagara and Yao 2007) as $\beta=r_{y} / r_{x}$ where $r_{x}$ and $r_{y}$ are axial lengths of the ellipse along the $x$ - and $y$-axis.

Figure 3-7 shows the square root of the derived reduced scattering coefficient perpendicular and parallel $\left(\sqrt{\mu_{s, 90}^{\prime} / \mu_{s, 0}^{\prime}}\right)$ for three different cylinder radii: $0.1,0.25$, and 1.5 $\mu m$ as a function of the fitted axes ratio $\beta$. The optical properties of the background were maintained at $\mu_{s, b}^{\prime}=40 \mathrm{~cm}^{-1}, g_{b}=0.8$, and $\mu_{a}=0.1 \mathrm{~cm}^{-1}$. The cylinder concentration $\left(c_{A}\right)$ in the medium was altered to change the reduced scattering coefficient ratios. It can be seen that the $\sqrt{\mu_{s, 90}^{\prime} / \mu_{s, 0}^{\prime}}$ is proportional (slope $=1.02$ ) to the axis ratio $\beta$ as predicted by the ADE. This is not surprising within the ADE framework, but it is clear that neither the axes ratio nor the cylinder scattering coefficient can be correctly interpreted by this theory although it can fit the time-resolved reflectance very well. 


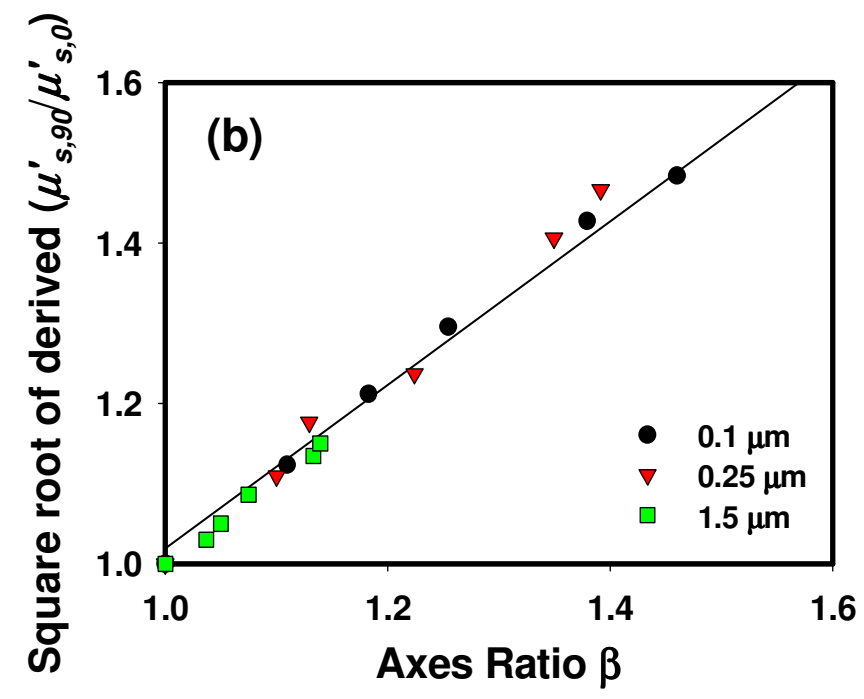

Figure 3-7: The square root of the derived reduced scattering coefficient perpendicular and parallel $\sqrt{\mu_{s, 90}^{\prime} / \mu_{s, 0}^{\prime}}$ for three different cylinder radii: $0.1,0.25$, and $1.5 \mu \mathrm{m}$ as a function of the fitted axes ratio $\beta$ of spatial resolved measurements.

In this study, some assumptions were made that are not quite the same in biological tissue. For instance, both the fiber alignment and fiber size have some variations and the absorption coefficient may not be homogenous in tissue. These issues need further study and clarification. However, we suspect the general conclusion is still valid that isotropic diffuse model cannot fully characterize fibrous samples unless the fiber size is determined. 


\section{CHAPTER 4}

\section{COMPARISON OF ISOTROPIC AND ANISTROPIC DIFFUSE MODELS IN DETERMINING OPTICAL PROPERTIES IN A FIBROUS MEDIUM USING TIME- RESOLVED REFLECTANCE}

\subsection{Introduction}

Tissue structural properties strongly affect how the photons are scattered. Knowledge of such interactions is essential for developing optical techniques to derive tissue structure properties, such as size, concentration, and alignment of fibers which strongly influence the tissue mechanical properties (Lake et al. 2009; Morgan et al. 2006; Parry and Craig 1984). Usually, light propagation in biological tissue is described by the radiative transport equation and tissues are assumed to be isotropic, i.e., their optical properties are independent of the measurement direction. However, this assumption is invalid when biological tissues contain ordered microstructures, such as ordered elongated subunit like the collagen fibers in skin (Nickell et al. 2000), dentin (Kienle et al. 2003), and tendon (Kienle et al. 2007). For instance, Marquez et al. (1998) used the spatial resolved technique and found that the optical properties of chicken breast were different when measured parallel or perpendicular to the fiber.

Optical properties can be derived by fitting time-resolved reflectance using the diffuse equation. The diffusion equation is an approximation to the more accurate radiative transfer equation. From the assumed boundary condition at the tissue-air interface, the complexity of the diffuse equation varies. For boundaries with mismatched 
refractive indices, the extrapolated-boundary condition (EBC) is usually used, which states that the fluence rate goes to zero at some distance beyond the actual surface (Haskell et al. 1994). A simpler solution that assumes a zero-boundary condition (ZBC) (Patterson et al., 1989) can be applied when the measurement is at large distance. Hielscher et al. (1995) found that the zero-boundary condition diffuse equation is capable of deriving the same scattering and absorption coefficient from the experimental data with almost the same accuracy as the more sophisticated solution using extrapolated boundary condition. Several studies used the zero-boundary condition to avoid the unnecessary complexity of the extrapolated boundary condition in deriving the optical properties of the tissue (Cubeddu et al. 1994).

Several methods have been developed to study light propagation in anisotropic media. Heino et al. (2003) replaced the diffuse scalar coefficient with a diffuse tensor in the diffusion equation to describe the anisotropic diffuse equation (ADE). Gandjbakhche derived analytical solution of light reflectance and transmittance based on the continuous time random walk (CTRW) in anisotropic medium (Dagdug et al. 2003). Both models correctly predicted the elliptical equi-intensity reflectance profiles in a medium that consists of cylindrical shape scatters (such as aligned fiber). This observation was confirmed experimentally in several anisotropic tissue models (Hebden et al. 2004; Ranasinghesagara et al. 2006; Ranasinghesagara and Yao 2007).

Kienle et al. (2004) developed a Monte Carlo simulation to simulate light propagation in medium that consists of aligned infinitely long cylinders. Kienle (2007) showed that the results obtained from ADE don't agree quantitatively with those obtained from the Monte Carlo simulation. In a recent study, it was shown that these two methods 
provide consistent results in samples of small fibers (Shuaib and Yao 2010). On the other hand, for time-resolved measurement, it was shown that derived reduced scattering coefficient using the isotropic diffuse model under extrapolated-boundary condition varied with the fiber direction (Kienle et al. 2007). The reduced scattering coefficient derived along the fiber direction was close to the background reduced scattering coefficient. However, this reduced scattering coefficient was larger than any that obtained along other directions. The reduced scattering coefficient was found to increase when the ratio of cylinder and background reduced scattering coefficient increases. In contrast, the derived absorption does not depend on the measurement direction, but to obtain correct values the distance between the incident light and the detector needs to be large ( $\rho>>$ $\left.1 / \mu_{s}^{\prime}\right)$ (Kienle et al. 2007). Shuaib et al. (2011) studied fiber size effect in applying the isotropic diffuse equation solution to derive optical properties from time-resolved measurement. The derived reduced scattering coefficient when measured perpendicularly to the fiber direction was found to be in good agreement with the summation of the reduced scattering coefficients of the background and the cylinder in a medium with small cylinder radii. Furthermore, knowledge of the fiber size in the medium is necessary to obtain the correct reduced scattering coefficient associated with larger cylinders.

In this study, we compared the use of isotropic and anisotropic diffuse models in retrieving the optical properties in fibrous samples from time-resolved reflectance measurements. A Monte Carlo model was used to simulate time resolved reflectance in a fibrous tissue consisting of aligned cylinders and spherical particles. Using the anisotropic diffuse model, the reduced scattering coefficients for both cylinder and background can be derived from a single time-resolved reflectance measured 
perpendicularly to the fiber direction. Using isotropic diffuse solution, these parameters can be derived from two measurements. The background reduced scattering coefficient is calculated by fitting the isotropic diffuse solution to the time-resolved reflectance measured along the fiber direction; whereas the summation of the reduced scattering coefficients of the background and cylinders can be derived by fitting the isotropic diffuse model to the reflectance measured perpendicularly to the fiber direction. The simulation results indicate that the derived optical properties are accurate when fiber diameter is small. For large fiber diameters, the reduced scattering coefficient of fibers needs to be corrected by fiber-size dependent factor.

\subsection{Method}

\subsubsection{Monte Carlo simulation}

A pencil beam $(\lambda=800 \mathrm{~nm})$ was incident on the semi-infinite fibrous medium as shown in Figure 3-1. A detail description of the Monte Carlo simulation process is described previously (Kienle et al. 2004; Shuaib and Yao 2010). The simulation used in this chapter is exactly the same as described in Chapter 3.

\subsubsection{Diffuse theory model}

The solutions of both isotropic and anisotropic diffusion theory under two commonly used (zero- and extrapolated-) boundary conditions are applied to obtain the optical properties from the time-resolved measurement (Kienle and Patterson 1997; Kienle et al. 2007). Isotropic diffuse equation under zero- boundary condition, $I D E-Z B C$, for timeresolved measurement is as the following: 


$$
R_{I D E-Z B C}(\rho, t)=\frac{M \mathrm{e}^{-\mu_{a} c t} e^{-\frac{1}{4 c t}\left(\frac{\rho^{2}}{D}\right)} z_{0} e^{-\frac{z_{0}^{2}}{4 D c t}}}{(4 \pi D c)^{3 / 2} t^{5 / 2}}
$$

where $D=1 /\left(3 \cdot\left(\mu^{\prime}{ }_{s}+\mu_{a}\right)\right)$ is the diffusion constant, $c$ is the speed of light in turbid medium, $M$ is a multiplicative constant. $\rho=\sqrt{x^{2}+y^{2}}$ is the measurement distance from the incident light, where $x$ and $y$ are the distance away from the incident light along the $x$ and $y$-axis on the sample surface, respectively. $\mu_{s}$ is the scattering coefficient, $g$ is the anisotropic factor, and $\mu_{s}^{\prime}$ is the reduced scattering coefficient and is $(1-g) \mu_{s} ; \quad z_{0}=1 / \mu_{s}^{\prime}$ is the transport mean free path.

Isotropic diffuse equation under extrapolated-boundary condition, $I D E-E B C$, for time-resolved measurement is as the following:

$$
R_{I D E-E B C}(\rho, t)=\frac{M \mathrm{e}^{-\mu_{a} c t} e^{-\frac{\rho^{2}}{4 D c t}}}{2(4 \pi D c)^{3 / 2} t^{5 / 2}} \times\left[z_{0} e^{-\frac{z_{0}^{2}}{4 D c t}}+\left(z_{0}+2 z_{b}\right) e^{-\frac{\left(z_{0}+2 z_{b}\right)^{2}}{4 D c t}}\right]
$$

where $z_{b}=2 A D, A=\left(1+R_{e f f}\right) /\left(1-R_{e f f}\right)$, and $R_{\text {eff }}$ is the fraction of photon that are internally diffusely reflected at the boundary (Haskell et al. 1994).

To obtain the anisotropic diffusion equation in the time-domain, a diffuse tensor was applied to describe the incident angle dependent diffusion process. It is assumed that only the diagonal component of $D$ is not zero. Different diffuse coefficients are applied: $D$ represents the diffuse along the fibers direction and $D_{\perp}$ represents that perpendicular to the fibers (along the $x$-axis, and $z$-axis). The anisotropic diffuse equation can be obtained as shown previously by Johnson et al. (2002) by applying to the solution of the isotropic diffusion equation a coordinate transformation: $x^{\prime}=\sqrt{D / D_{\perp}} x$, where the 
anisotropic diffuse equation under the zero-boundary condition, $A D E-Z B C$, for timeresolved measurement is :

$$
R_{A D E-Z B C}(x, y, t)=\frac{M \mathrm{e}^{-\mu_{a} c t} e^{-\frac{1}{4 c t}\left(\frac{x^{2}}{D_{\perp}}+\frac{y^{2}}{D}\right)} z_{0} e^{-\frac{z_{0}^{2}}{4 D c t}}}{(4 \pi D c)^{3 / 2} t^{5 / 2}}
$$

And the anisotropic diffuse equation under extrapolated boundary condition, $A D E-E B C$, for time-resolved measurement is:

$$
R_{A D E-E P C}(x, y, t)=\frac{M \mathrm{e}^{-\mu_{a} c t} e^{-\frac{1}{4 c t}\left(\frac{x^{2}}{D_{\perp}}+\frac{y^{2}}{D}\right)}}{2(4 \pi D c)^{3 / 2} t^{5 / 2}} \times\left[z_{0} e^{-\frac{z_{0}^{2}}{4 D c t}}+\left(z_{0}+2 z_{b}\right) e^{-\frac{\left(z_{0}+2 z_{b}\right)^{2}}{4 D c t}}\right]
$$

where $D_{\perp}=1 /\left(3 \cdot\left(\mu^{\prime}{ }_{s, c}+\mu^{\prime}{ }_{s}+\mu_{a}\right)\right)$ is the diffusion constant. To be noted, when the reflectance measurement is parallel to the fibers $(x=0)$, the $R_{A D E}$ becomes the same as $R_{I D E}$.

The Levenberg-Marqurdt nonlinear fitting algorithm with MultiStart (Global Optimization Toolbox 3.0) in MATLAB (The Mathworks Inc., Natick, MA) was applied to retrieve the optical properties using the different diffuse equations. The range of the fitting for the leading and tailing data was $85 \%$ and $0.1 \%$ of the maximum value, respectively. For the uncertainty in the fitting, the confidence intervals calculated using MATLAB utilized the derived parameters: coefficient estimates, residuals, and the Jacobian matrix from the nonlinear fitting algorithm.

When the isotropic diffuse equations were applied, two measurements, parallel and perpendicular to the fiber direction, were used to derive the optical properties of the fibrous tissue. The derived reduced scattering and absorption coefficients when the measurement is parallel $\left(0^{\circ}\right)$ and perpendicular $\left(90^{\circ}\right)$ to the cylinder direction are defined 
as $\mu_{s, 0}^{\prime}, \mu_{a, 0}$ and $\mu_{s, 90}^{\prime}, \mu_{a, 90}$, respectively. When the anisotropic diffuse equations were applied, only the time-resolved reflectance measured perpendicular to the fiber direction was used to derive the optical properties of the tissue. Therefore, the derived reduced scattering coefficients $\mu_{s, 0}^{\prime}$ and $\mu_{s, 90}^{\prime}$ are calculated from the diffuse constants $D$ and $D_{\perp}$, respectively. And only one absorption coefficient was retrieved and represented as $\mu_{a}$.

\subsection{Results}
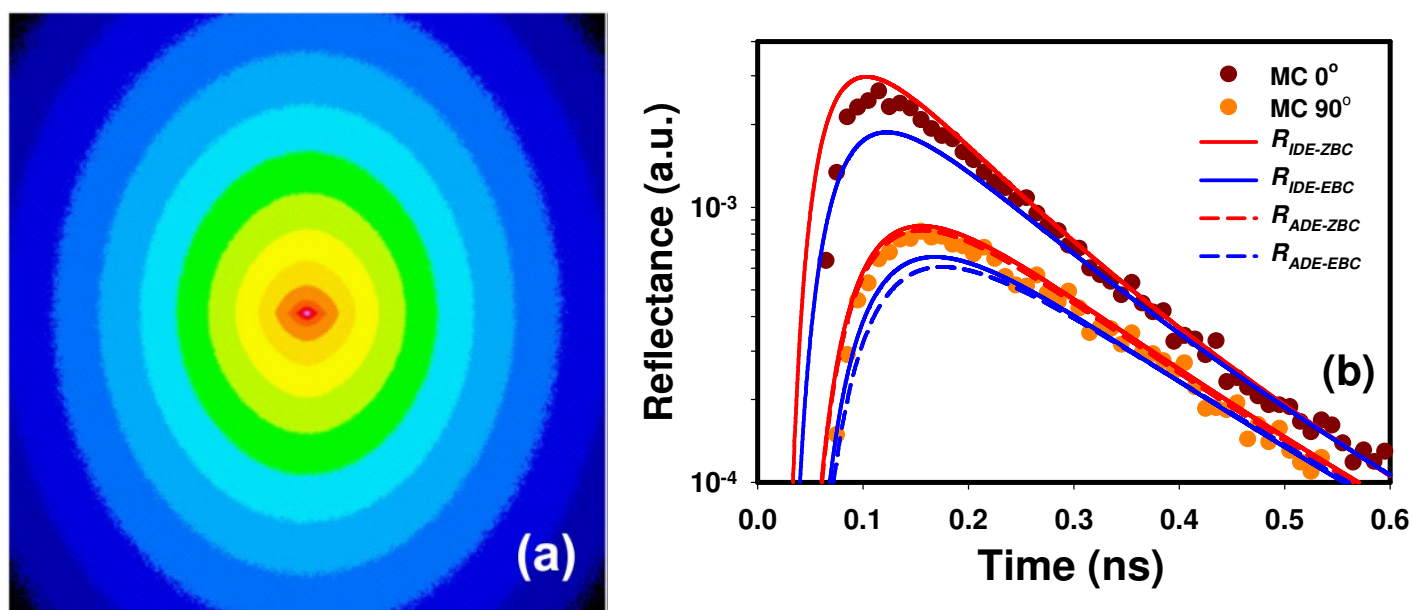

Figure 4-1: (a) Sample spatial resolved reflectance image obtained in anisotropic medium. The cylinder was aligned along the $y$-axis. The image size is $1 \times 1 \mathrm{~cm}^{2}$. (b) The time resolved measurement parallel $\left(0^{\circ}\right)$ and perpendicular $\left(90^{\circ}\right)$ to the fiber direction (solid lines) at $\rho=0.975$ $\mathrm{cm}$ and the reflectance predicted by (a) $R_{I D E-Z B C}$, (b) $R_{I D E-E B C}$, (c) $R_{A D E-Z B C}$, and (d) $R_{A D E-E B C}$. The optical properties used in the MC are $\mu_{s, c}^{\prime}=5.0 \mathrm{~cm}^{-1}, \mu_{a}=0.1 \mathrm{~cm}^{-1}, g=0.8, \mu_{s, b}^{\prime}=8.0 \mathrm{~cm}^{-1}$, and $r$ $=0.1 \mu \mathrm{m}$.

Figure 4-1(a) shows an example of the spatial-resolved reflectance image of an anisotropic medium where the cylinder structures were aligned along the $y$-axis. The cylinders had a radius of $r=0.1 \mu \mathrm{m}$, a refractive index of $n_{c}=1.46$ and a background refractive index of $n_{b}=1.36$. When the incident light is perpendicular to the cylinder $(\xi=$ $\left.90^{\circ}\right)$, the corresponding scattering coefficient was $\mu_{s, c}=6.665 \mathrm{~cm}^{-1}$ and $g_{c}=0.2498$. The 
background spherical scatters had $\mu_{s, b}=40 \mathrm{~cm}^{-1}$ and $g_{b}=0.8$. The absorption coefficient was $\mu_{a}=0.1 \mathrm{~cm}^{-1}$. It can be seen in Figure 4-1(a) that at small distance away from the incident light, the equi-intensity has an elliptical shape that is elongated perpendicular to the fiber direction. As the distance away from the incident light increases, the equiintensity goes through a transition, where the shape changes continuously. However, at large distance from the incident light, the equi-intensity shaper becomes elliptical that is elongated parallel to the fiber direction.

Figure 4-1(b) shows the time-resolved reflectance measurements parallel $\left(0^{\circ}\right)$ and perpendicular $\left(90^{\circ}\right)$ to the fiber direction at $\rho=0.975 \mathrm{~cm}$. Also, the prediction isotropic and anisotropic diffuse under zero- and extrapolated-boundary condition are shown in Figure 4-1(b). For the reflectance parallel to the fiber direction $\left(0^{\circ}\right)$, it can be seen for times longer than $\sim 0.3 n s$ the diffusion theories and Monte Carlo simulation agree very well. However, at earlier times, $R_{I D E-Z B C}$ overestimates the reflectance, whereas, $R_{I D E \_E B C}$ underestimate the reflectance. To be noted, the anisotropic diffuse equation becomes the isotropic diffusion equation when the detector is placed parallel to the fiber direction; therefore, only the $R_{I D E}$ predictions are shown at $0^{\circ}$ in Figure 4-1(b). For the reflectance perpendicular to the fiber direction $\left(90^{\circ}\right)$, it can be seen that both $R_{I D E-Z B C}$ and $R_{A D E-Z B C}$ under zero-boundary condition slightly overestimate the reflectance but $R_{A D E-Z B C}$ is closer to the reflectance. However, $R_{I D E-E B C}$ slightly underestimates the reflectance, while the $R_{A D E-E B C}$ significantly underestimates the reflectance. All diffuse models poorly predict the photons at earlier times.

The different diffuse models were applied to retrieve the optical properties from time-resolved reflectance measurement of tissues at $\rho=0.975 \mathrm{~cm}$ with different cylinder 
radii: $0.1,0.25$, and $1.5 \mu \mathrm{m}$ with different total reduced scattering coefficients $\left(\mu_{s, t}\right)$. The reduced scattering coefficient of the cylinders $\left(\mu_{s, c}^{\prime}\right)$ ranged from 2 to $10 \mathrm{~cm}^{-1}$. All the sample background reduced scattering coefficient $\left(\mu_{s, b}^{\prime}\right)$ were maintained at a constant value of $8 \mathrm{~cm}^{-1}$; the background anisotropy $g_{b}$ is 0.8 , and the absorption coefficient $\mu_{a}$ is $0.1 \mathrm{~cm}^{-1}$.
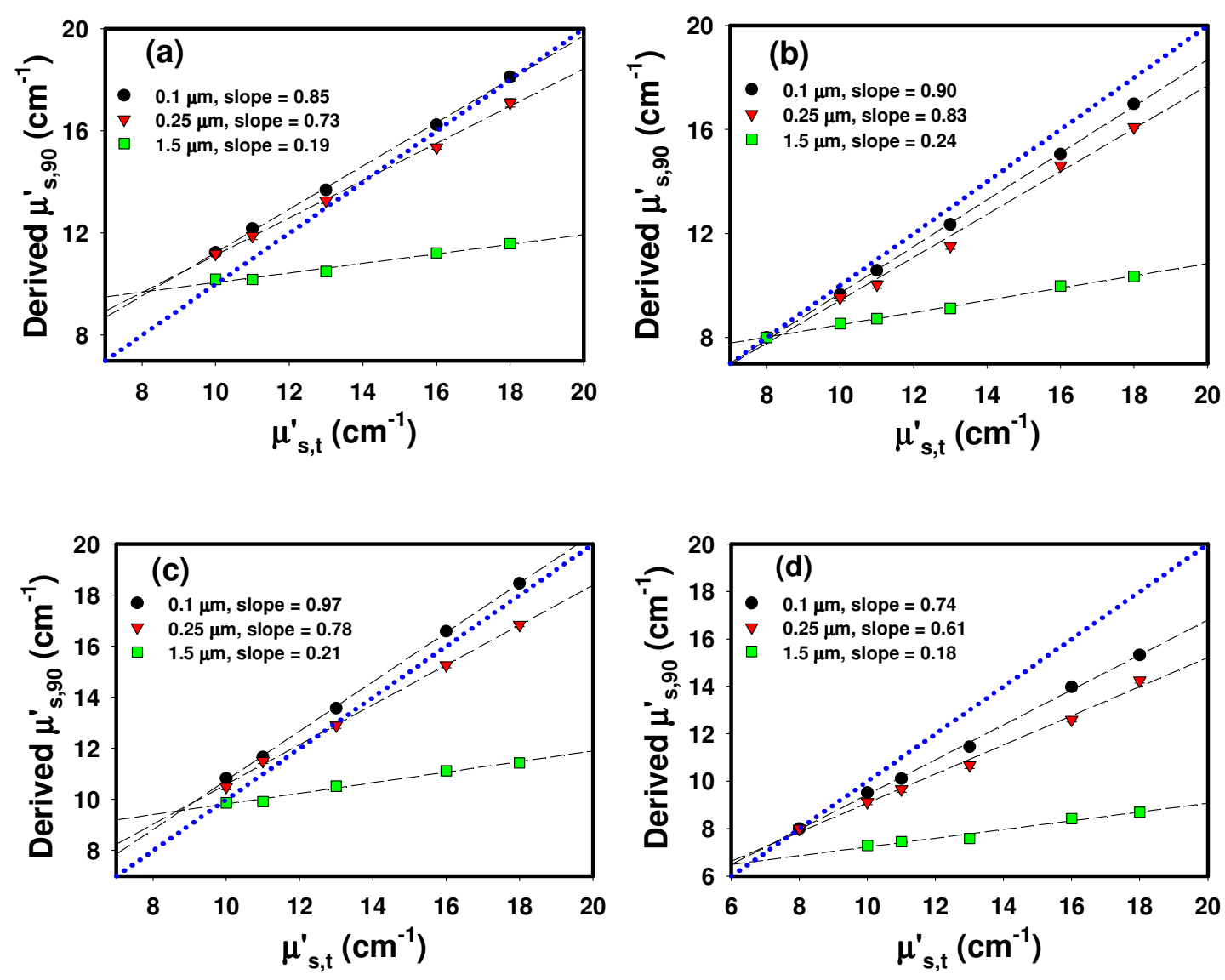

Figure 4-2: The derived reduced scattering coefficient perpendicular to the cylinder direction from using (a) $R_{I D E-Z B C}$, (b) $R_{I D E-E B C}$, (c) $R_{A D E-Z B C}$, and (d) $R_{A D E-E B C}$ for different cylinder radii: 0.1 , $0.25,1.5 \mu \mathrm{m}$ with different total reduced scattering coefficients $\mu_{s, t}$. The dotted lines indicate the theoretical values. Error bars represent the $95 \%$ c.i. of the fitted values. In here, the symbol is larger than the error bars.

Figure 4-2 shows the derived the reduced scattering coefficient perpendicular to the fiber direction $\mu_{s, 90}^{\prime}$ from (a) $R_{I D E-Z B C}$, (b) $R_{I D E-E B C}$, (c) $R_{A D E-Z B C}$, and (d) $R_{A D E-E B C}$ to 
the cylinder direction for different cylinder radii, $r$, with different total reduced scattering coefficients $\left(\mu_{s, t}^{\prime}\right)$. For all the different models, the derived $\mu_{s, 90}^{\prime}$ shows a strong dependency on the cylinder size. Also, the different models increased linearly with the total reduced scattering coefficient $\left(\mu_{s, t}^{\prime}\right)$. The different size of cylinder $0.1,0.25$, and 1.5 $\mu m$ had different linear slopes for different diffuse models, i.e., $0.85,0.73$, and 0.19 for $I D E-Z B C, 0.90,0.81$, and 0.24 for $I D E-E B C, 0.73,0.61$, and 0.18 for $A D E-Z B C$, and $0.73,0.61$, and 0.18 for $A D E-E B C$, respectively.

It can be seen that the derived reduced scattering coefficients for all different diffuse equations except for the ADE-EBC were close to the true value when the cylinder size is small, whereas the ADE-EBC was greatly underestimated for all the cylinder sizes. For small total reduced scattering coefficients, IDE-ZBC greatly overestimated the reduced scattering coefficient for small cylinder sizes. 

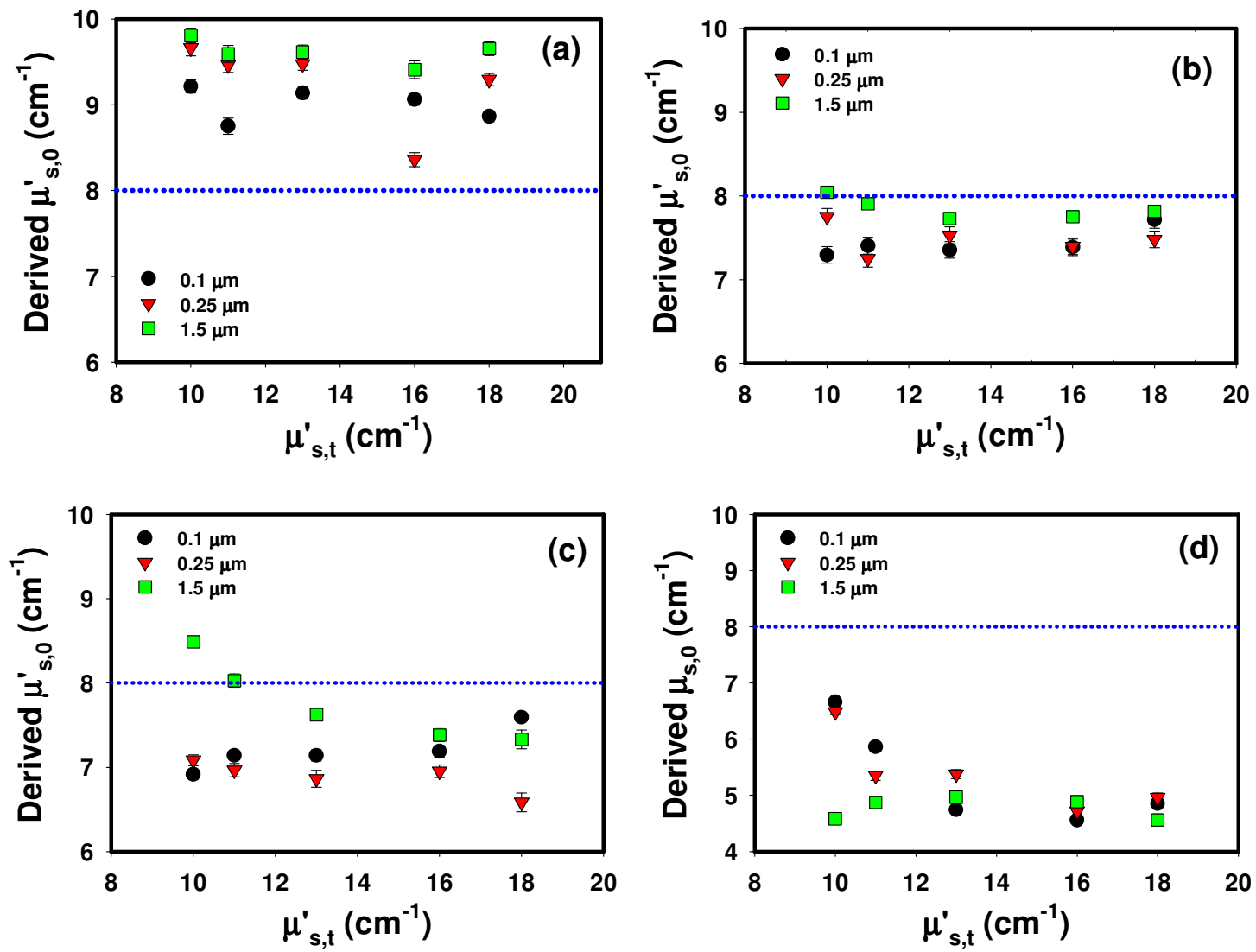

Figure 4-3: The derived reduced scattering coefficients parallel to the cylinder direction are shown using (a) $R_{I D E-Z B C}$, (b) $R_{I D E-E B C}$, (c) $R_{A D E-Z B C}$, and (d) $R_{A D E-E B C}$ for different cylinder radii: $0.1,0.25$, and $1.5 \mu \mathrm{m}$ with different total reduced scattering coefficients $\mu_{s, t}$. The dotted lines indicate the theoretical values. Error bars represent the $95 \%$ c.i. of the fitted values.

Figure 4-3 shows the derived reduced scattering coefficients parallel $\mu_{s, 0}^{\prime}$ to the cylinder direction using (a) $R_{I D E-Z B C}$, (b) $R_{I D E-E B C}$, (c) $R_{A D E-Z B C}$, and (d) $R_{A D E-E B C}$ for different cylinder radii, $r$, with different total reduced scattering coefficients $\left(\mu_{s, t}\right)$. For IDE-ZBC, the derived reduced scattering coefficients were overestimated. For the IDEEBC and ADE-ZBC, the reduced scattering coefficients were close to the true value of the background reduced scattering coefficient. However, the derived reduced scattering coefficients obtained by using the ADE-EPC were significantly underestimated. The 
error in $\mu_{s, b}^{\prime}$ for the different models are $22.6 \%$ for the IDE-ZBC, $9.4 \%$ for the IDEEBC, $18 \%$ for the ADE-ZBC, and $42.98 \%$ for the ADE-EBC.
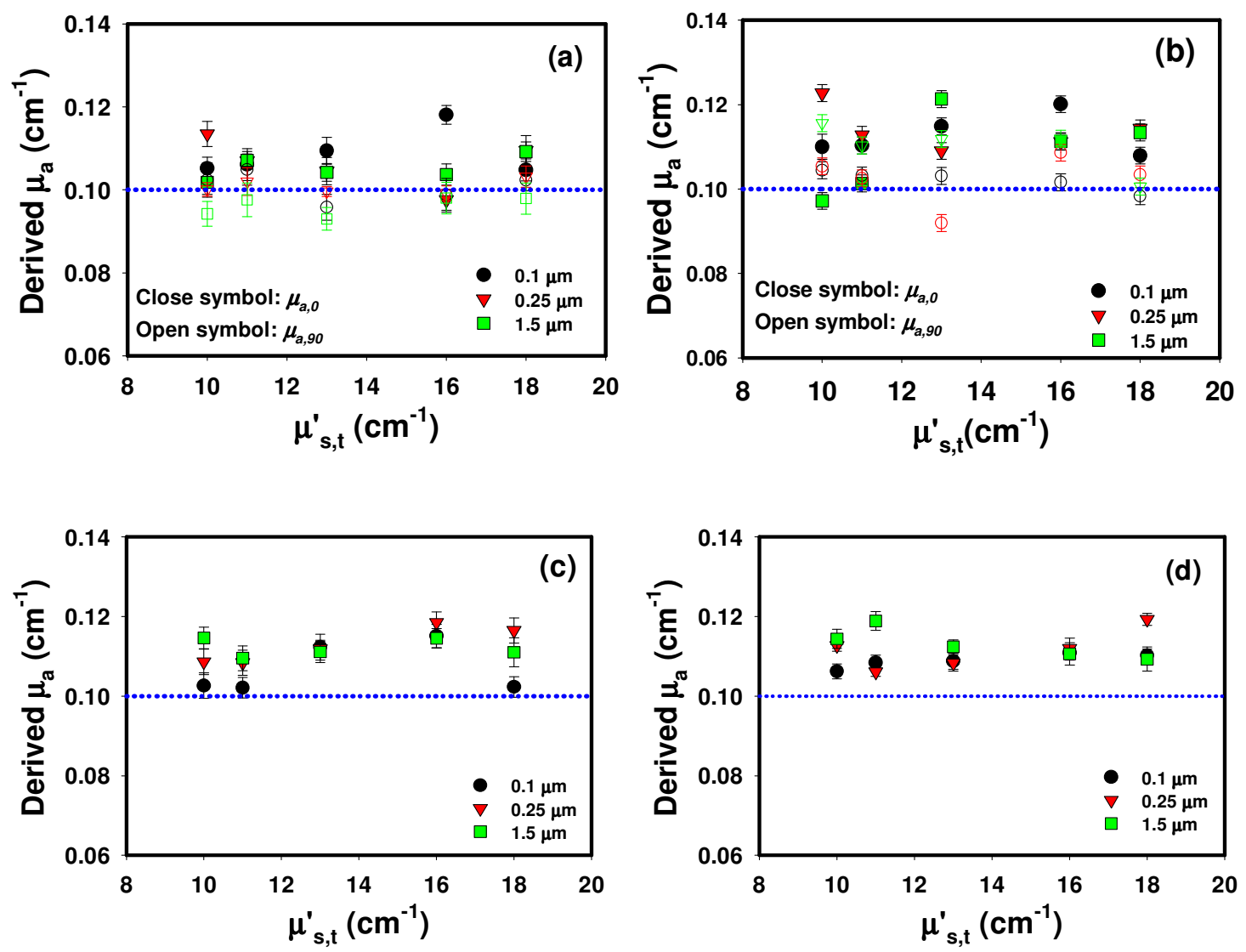

Figure 4-4: The derived absorption coefficient from using (a) $R_{I D E-Z B C}$, (b) $R_{I D E-E B C}$, (c) $R_{A D E-Z B C}$, and (d) $R_{A D E-E B C}$ for different cylinder radii: $0.1,0.25$, and $1.5 \mu \mathrm{m}$ with different total reduced scattering coefficients $\mu_{s, t}^{\prime}$. The dotted lines indicate the theoretical values. Error bars represent the $95 \%$ c.i. of the fitted values.

Figure 4-4 shows the derived the absorption coefficients: $\mu_{a, 0}$ and $\mu_{a, 90}$ from (a) $R_{I D E-Z B C},(\mathrm{~b}) R_{I D E-E B C}$, and the absorption coefficients: $\mu_{a}$ from (c) $R_{A D E-Z B C}$, and (d) $R_{A D E-}$ $E B C$ to the cylinder direction for different cylinder radii, $r$, with different total reduced scattering coefficients $\left(\mu_{s, t}^{\prime}\right)$. All the models shows that the absorption coefficients are independent of the measurement direction; the errors in $\mu_{a}$ for the different models are less than $18.1 \%$ for IDE-ZBC, $22.3 \%$ for IDE-EBC, $18.5 \%$ for the ADE-ZBC, and 
$19.4 \%$ for the ADE-EBC. Part of error is due to the fairly large noise in the measurement, which can be reduced by significantly increasing the simulated photons.
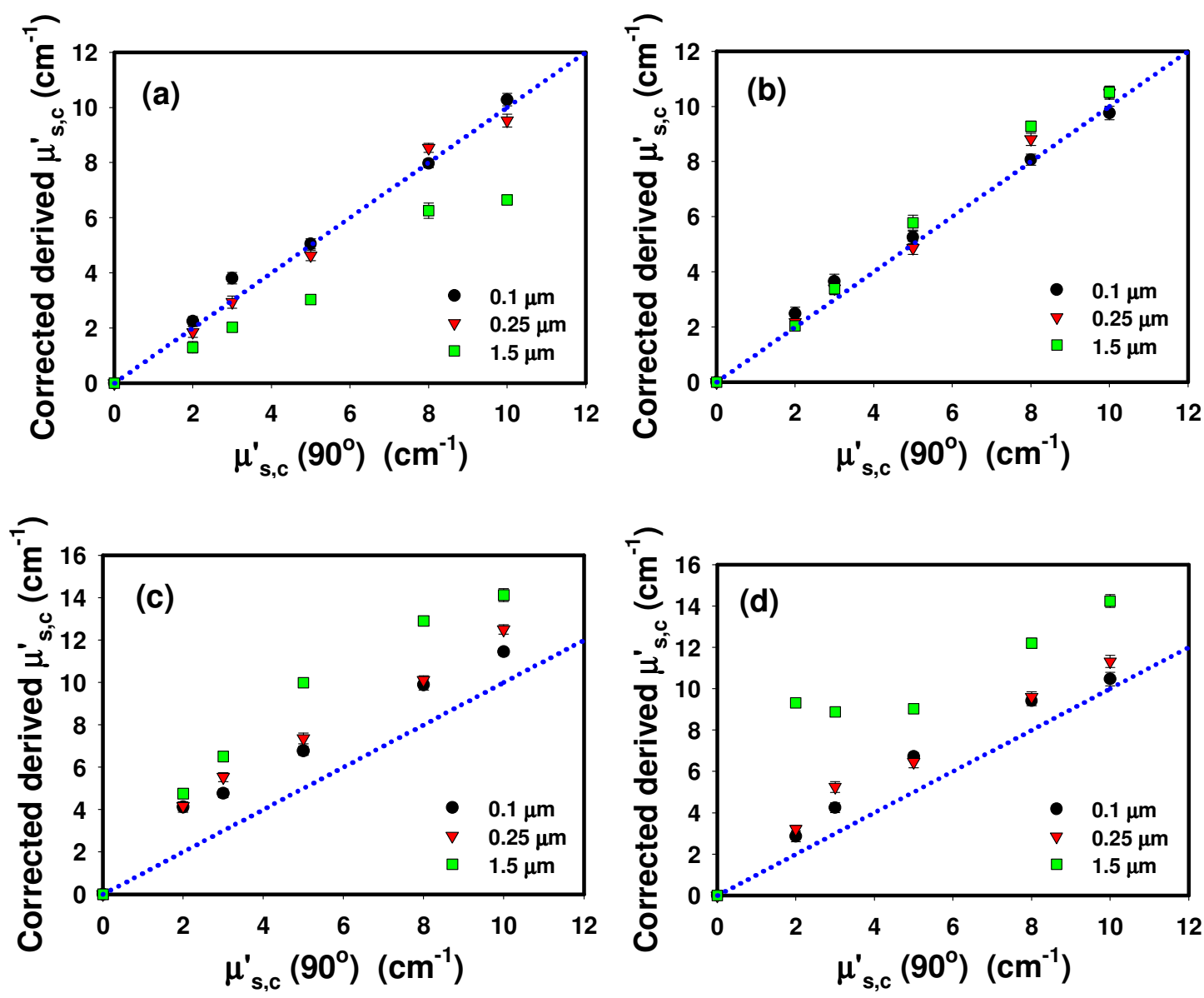

Figure 4-5: The corrected reduced scattering coefficients associated with the cylinder $\mu_{s, c}^{\prime}$ obtained by the different models: (a) $R_{I D E-Z B C}$, (b) $R_{I D E-E B C}$, (c) $R_{A D E-Z B C}$, and (d) $R_{A D E-E B C}$ using the Eq. (2-6) compared with the true cylinder reduced scattering coefficients $\mu_{s, c}^{\prime}\left(90^{\circ}\right)$. The dotted lines indicate the theoretical values. Error bars represent the $95 \%$ c.i. of the fitted values.

To further compare the models, the reduced scattering coefficients associated with the cylinder that is mentioned in the previous study (Shuaib and Yao 2011) can be derived assuming knowledge of the fiber size using the following equation: 


$$
\begin{gathered}
\mu_{s, 0}^{\prime}=\mu_{s, b}^{\prime} ; \mu_{s, 90}^{\prime}=\mu_{s, b}^{\prime}+\mu_{s, c}^{\prime}\left(90^{o}\right) \\
\text { Corrected } \mu_{s, c}^{\prime}\left(90^{o}\right)=\frac{\mu_{s, 90}^{\prime}-\mu_{s, 0}^{\prime}}{C(r)}=\frac{\mu_{s, c}^{\prime}\left(90^{o}\right)}{C(r)}
\end{gathered}
$$

where $\mathrm{C}(r)$ is a fiber-size dependent factor correction coefficient that is obtained from the slope of each cylinder radii in Figure 2-3. It can be seen from Figure 4-5 that the IDE$\mathrm{EBC}$ is the only diffuse equation that is capable to correctly derived reduced scattering associated with cylinder, where the error is less than $10 \%$.

\subsection{Discussion and conclusion}

In this study, light propagation in fibrous tissue that contains aligned cylinder and spherical shape scatters were simulated using a Monte Carlo model. We compared the isotropic and anisotropic diffuse models under two commonly used (zero- and extrapolated-) boundary conditions in retrieving the optical properties in fibrous samples from time-resolved reflectance measurements.

The surface reflectance in fibrous turbid medium was predicted from both ADE and CTRW to have an elliptical shape at large distance that is elongated along the fiber direction where the reduced scattering coefficient is the smallest. Also, the ADE predicted that the axes ratio of the elliptical shape is equal to the inverse of the square root of the corresponding reduced scattering coefficient parallel and perpendicular to the fiber direction. The axes ratio can be calculated using the method described previously as $\beta=r_{y} / r_{x}$, where $r_{x}$ and $r_{y}$ are axial lengths of the ellipse along the $x$ - and $y$-axis.

Figure 4-6 shows the square root of the derived reduced scattering coefficient perpendicular and parallel $\left(\sqrt{\mu_{s, 90}^{\prime} / \mu_{s, 0}^{\prime}}\right)$ using (a) $R_{I D E-Z B C}$, (b) $R_{I D E-E B C}$, (c) $R_{A D E-Z B C}$, and (d) $R_{A D E-E B C}$ for three different cylinder radii: $0.1,0.25$, and $1.5 \mu \mathrm{m}$ as a function of the fitted 
axes ratio $\beta$. The optical properties of the background were maintained at $\mu_{s, b}^{\prime}=40 \mathrm{~cm}^{-1}$, $g_{b}=0.8$, and $\mu_{a}=0.1 \mathrm{~cm}^{-1}$. The cylinder concentration $\left(c_{A}\right)$ in the medium was altered to change the reduced scattering coefficient ratios. Both the IDE-ZBC and IDE-EBC, the $\sqrt{\mu_{s, 90}^{\prime} / \mu_{s, 0}^{\prime}}$ is proportional (where the slope $=0.98$ for IDE-ZBC and 1.02 for IDE-EBC) to the axis ratio $\beta$ as predicted by the ADE. For ADE-ZBC and ADE-EBC, the slopes are 1.09 and 1.16, respectively. For both anisotropic models, it can be seen from Figure 46(c-d) that most of the points are not on the line. The ADE models are less consistent because of using only one measurement to derive the two reduced scattering coefficients, which might increase the error in the retrieved optical properties. These results conclude that even with the modification of the ADE, it still cannot correctly interpret the cylinder scattering coefficient. The IDE-EBC slope is the closest to the results obtained from the axes ratio. 

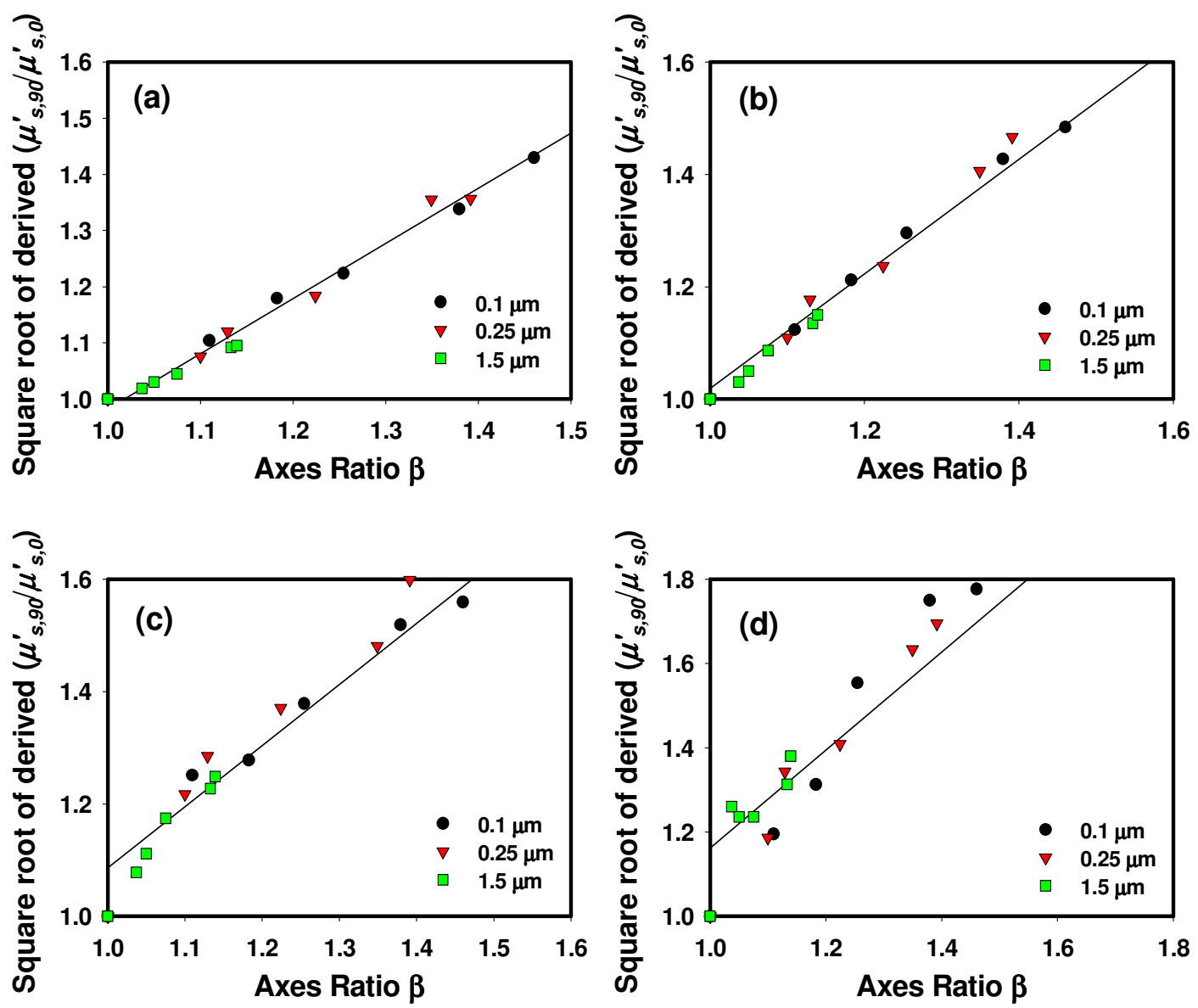

Figure 4-6: The square root of the derived reduced scattering coefficient perpendicular and parallel $\sqrt{\mu_{s, 90}^{\prime} / \mu_{s, 0}^{\prime}}$ using different models: (a) $R_{I D E-Z B C}$, (b) $R_{I D E-E B C}$, (c) $R_{A D E-Z B C}$, and (d) $R_{A D E-E B C}$ for three different cylinder radii: $0.1,0.25$, and $1.5 \mu \mathrm{m}$ as a function of the fitted axes ratio $\beta$ of spatial-resolved reflectance measurements.

The isotropic and anisotropic diffuse equations with different boundary conditions were not able to accurately predict the reduced scattering coefficient of fibrous tissue if the fiber size is large. However, the absorption coefficient can be extracted for fibrous tissue with reasonable accuracy Overall, the isotropic diffuse model produced better results than the anisotropic model. 


\section{CHAPTER 5}

\section{MEASURING PATH-LENGTH RESOLVED REFLECTANCE USING LOW-COHERENCE MACH- ZEHNDER INTERFEROMETRY ${ }^{3}$}

\subsection{Introduction}

Photon migration in tissue can be described by radiative transport theory or its diffuse approximation (Farrell et al. 1992). In many studies, biological tissues can be treated as an isotropic turbid medium where light scattering is independent of incident direction. However, this treatment is invalid in anisotropic tissues such as tendon (Kienle et al. 2004), dentin (Kienle et al. 2003), skin (Nickell et al. 2000) and skeletal muscle (Ranasinghesagara and Yao 2007). Since these anisotropic tissues are abundant in humans and animals, a thorough understanding on light propagation in such tissues is critical for correctly interpreting optical measurements obtained therein.

Anisotropic tissues, such as tendon, usually have a predominant axis that is determined by the fibrous collagen structures. Nickell et al. (2000) found that the equiintensity reflectance distribution at skin surface resembled an ellipse whose long axis was perpendicular to the collagen fiber orientation at small detection distances, but parallel to the fibers at larger distances. Kienle et al. (2007) found that the reduced optical scattering coefficient was higher when measured perpendicularly to the fibers. Both anisotropic diffuse equation (ADE) (Johnson et al. 2002) and continuous time random walk (CTRW)

\footnotetext{
${ }^{3}$ The Chapter is from a published paper. C. Fan*, A. Shuaib*, and G. Yao "path-length resolved reflectance in tendon and muscle" Opt. Express 19, 8879-8887 (2011).
} 
theory (Dudko et al. 2004) predicted the elliptical shape of the equi-intensity reflectance in fibrous samples and its alignment with fiber orientations, which have been further confirmed in several additional experimental studies (Hebden et al. 2004; Ranasinghesagara et al. 2006; Sviridov et al. 2005).

Skeletal muscles are the other major anisotropic tissues in human and are essential for many important physiological functions. In addition to their fibrous appearance, skeletal muscle fibers are composed of repetitive units called "sarcomere" which are the fundamental structural and contractile units in striated muscle (Lieber 2002). In fact, many muscle diseases are associated with mutations in sarcomeric proteins (Laing and Nowak 2005). Light propagation in striated muscle was found to be affected by the sarcomere structures (Ranasinghesagara and Yao 2008; Xia et al. 2006), and a unique 2D reflectance pattern was documented in skeletal muscles (Ranasinghesagara and Yao 2007). Subsequent studies have revealed that different striated muscles have somewhat different 2D reflectance patterns due to variations in muscles' structural properties (Ranasinghesagara et al. 2010).

Time or path-length resolved optical measurements have been widely used to study light propagation in tissue (Delpy et al. 1988). In addition to the use of short-pulse lasers, an alternative implementation is to use "coherence gating" based on low coherence interferometry (LCI) to achieve path-length-resolved measurements. Popescu \& Dogariu (1999) measured optical reflectance in scattering samples using a Michelsonbased interferometric implementation of LCI and found that experimental measurements can be described by a time-resolved diffuse model. Mach-Zehnder-based LCI (Varghese 
et al. 2007) was also applied to study path-length resolved Doppler shift of multiple scattered photons in turbid media.

In this study, we used a single-mode fiber optic Mach-Zehnder LCI to investigate the angular-dependent light propagation in anisotropic tissues including tendon and skeletal muscle. Measurements were conducted at different distances from the incidence and different angles from the fiber orientation in tissue. The experimental and measurement details are described in Section 5.2. Section 5.3 shows the measured reflectance data which revealed both similarities and significant differences in tendon and skeletal muscle. In the discussion section (Section 5.4), the authors reanalyzed the results shown in Section 5.3 and shows that anisotropic diffuse theory can explain this dissertation's finding concerning light propagation in tendon, but not in skeletal muscle.

\subsection{Materials and methods}

Fresh bovine skeletal muscle and tendon samples were obtained from the meat science laboratory at the University of Missouri-Columbia. All surface fat on the tissue samples were carefully removed before measurements were taken. 


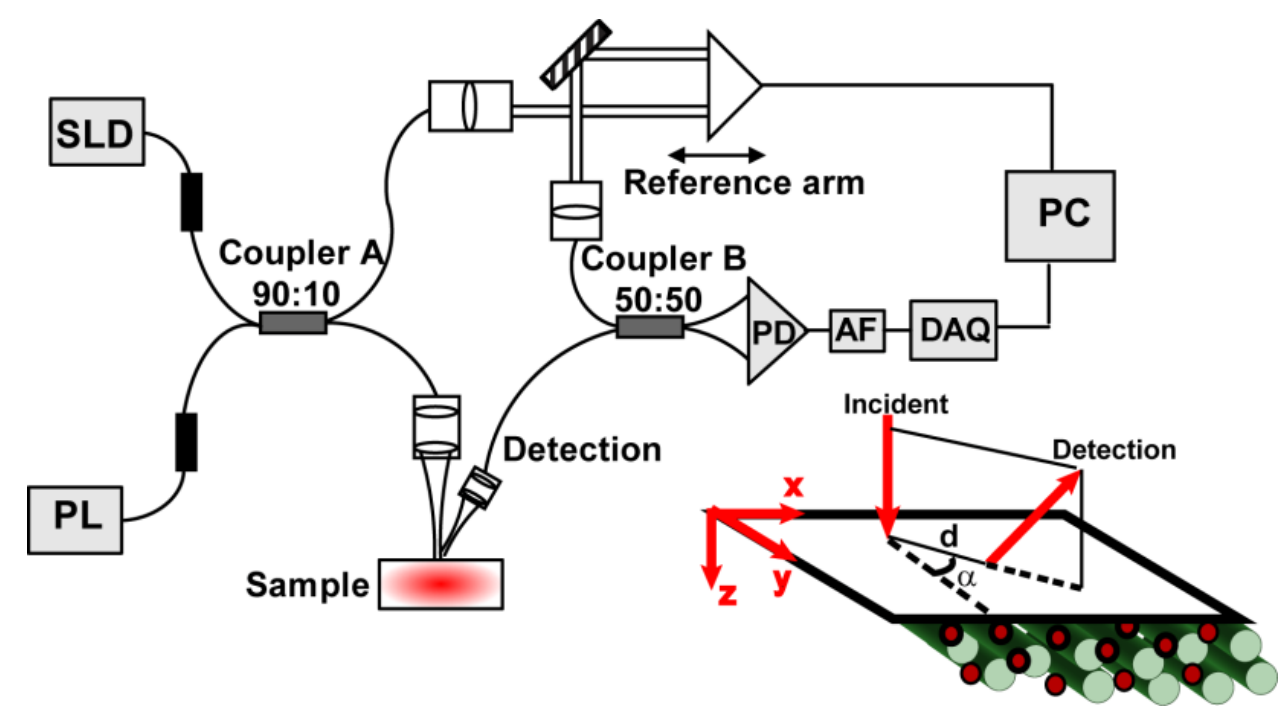

Figure 5-1: This schematic diagram of the fiber optic Mach-Zehnder low coherence interferometer shows. SLD, superluminescent diode; PL, pilot light for aiming; PD, balance photo-detector; AF, amplifier \& band-pass filter; and DAQ, data acquisition board.

Tissue samples were scanned by using a single mode fiber optic Mach-Zehnder interferometer (Fig. 5-1) that is similar to those reported previously (Varghese et al. 2007). The light source was a $4 \mathrm{~mW}$ superluminescent diode (SLD-561, Superlum Inc., Russia) with a central wavelength of $\lambda_{\mathrm{o}}=1287.0 \mathrm{~nm}$ and spectral bandwidth $\Delta \lambda_{\mathrm{FWHM}}=$ $32.7 \mathrm{~nm}$ (with calculated coherence length of $22.4 \mu \mathrm{m}$ in air). The $4 \mathrm{~mW}$ light was split by the fiber coupler A with $90 \%$ of the source power delivered to the sample, and the remaining $10 \%$ used as the reference light. In the sample arm, the incident light was focused onto the sample by using an $f=25 \mathrm{~cm}$ lens which produced a $41 \mu \mathrm{m}$ diameter incident spot. The detection head was angled at $60^{\circ}$ from the sample surface and consisted of two identical lenses $(f=8 \mathrm{~mm}$, N.A. $=0.28)$, resulting in a detection diameter of $9.4 \mu \mathrm{m}$ on the sample surface. The collected photons were coupled into a $2 \times 2$ singlemode fiber coupler B. The reference light from the coupler A was reflected back from a retro-reflector and also coupled into the coupler B. A translational stage was used to 
move the retro-reflector to vary the path-length of the reference arm. Figure 5-2 shows the reference intensity for the reference arm when varying the reference arm path-length. The interference fringes were divided into two equal parts with a $\pi$ phase difference (Choma et al. 2003) which were detected by using a balanced photo-detector (2117-FC, New Focus Inc., USA). The signal was amplified, band-pass filtered and acquired by a data acquisition card into a computer for processing. The system had a detection sensitivity of $-123 \mathrm{~dB}$.

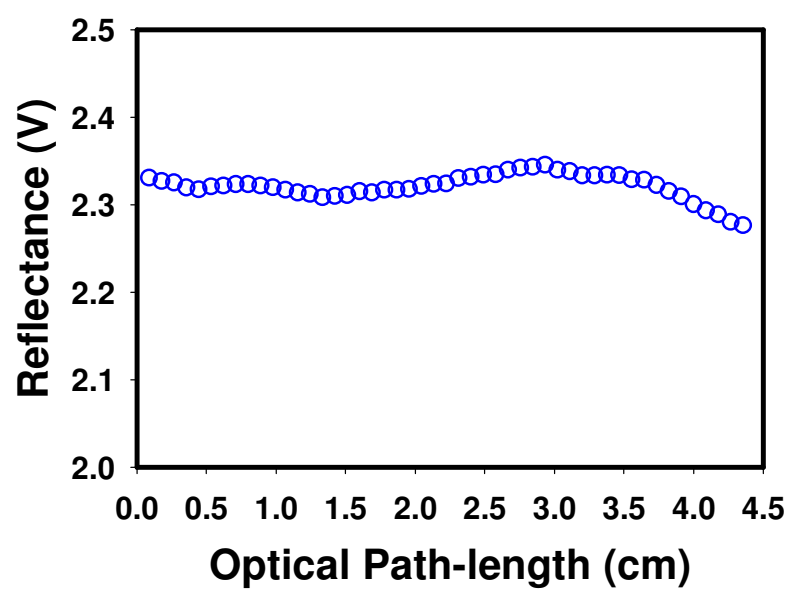

Figure 5-2: The reference intensity for the reference arm was recorded at different path-length arm distances, which is measured by a photo-detector (2011-FC, New Focus Inc., USA).

For convenience, a reference coordinate was defined with the $x-y$ plane on the sample surface as shown in Fig. 5-1. A focused incident beam was perpendicular to the sample surface with its incident location defined as the origin $(0,0)$. Tissue samples were positioned so that the y-axis was aligned with the orientation of fibers in the sample. Two parameters $(d$ and $\alpha$ ) were used to define the measurement position on the sample surface. The measurement distance $(d)$ was defined as the distance between detection 
position and the origin; whereas the measurement angular position $(\alpha)$ was defined as the angle formed between the measurement plane and sample fiber orientation (y-axis).

The sample was placed on a rotational stage so that the orientation $(\alpha)$ of the fibers could be adjusted. A cover glass was placed on the sample surface to define the measurement surface and tissues samples were in touch with the cover glass during the measurement. The detection fiber was scanned across the sample to measure reflectance at different distances $(d)$ away from the origin with a step size of $0.05 \mathrm{~cm}(e . g ., 0.00$, $0.05,0.10,0.20 \mathrm{~cm})$ and at different angular positions $\left(\alpha=0^{\circ}, 22.5^{\circ}, 45^{\circ}, 67.5^{\circ}, 90^{\circ}\right)$ relative to the fiber orientation. The maximal scanning distance of the reference arm was $1.1 \mathrm{~cm}$ (corresponding to a $2.2 \mathrm{~cm}$ path-length in air) where detected signals reached the noise floor. At every measurement position, eight measurements were repeated and averaged to improve the signal-to-noise ratio.

The detected signal at each detection position $(d, \alpha)$ is $S_{d, \alpha}(l) \propto 2\left(I_{r} I_{s}\right)^{1 / 2}$, where $I_{\mathrm{r}}$ and $I_{\mathrm{s}}$ are light intensities from the reference arm and sample arm, respectively. The effects of detector (PD in Fig.5-1) responsivity and amplifier gain (AF in Fig. 5-1) were removed from the acquired raw signals. The optical path-length resolved reflectance $r_{d, \alpha}(l)$ measured at distance $d$ and orientation angle $\alpha$ were calculated as: $r_{d, a}(l)=S_{d, \alpha}^{2}(l) /\left(4 I_{r} I_{i n}\right)$, where $I_{\text {in }}$ is the incident light intensity.

The total reflectance $R_{d, \alpha}$ and the mean path-length $\left\langle l_{d, \alpha}\right\rangle$ at position (d, $\left.\alpha\right)$ were calculated as:

$$
\begin{gathered}
R_{d, \alpha}=\frac{1}{N} \sum_{l=0}^{l_{\text {max }}} r_{d, \alpha}(l), \\
\left\langle l_{d, \alpha}\right\rangle=\sum_{l=0}^{l_{\text {max }}} l \cdot r_{d, \alpha}(l) / \sum_{l=0}^{l_{\text {max }}} r_{d, \alpha}(l),
\end{gathered}
$$


where $l$ is the optical path-length $\left(l_{\max }=2.2 \mathrm{~cm}\right.$ in air). The total reflectance was normalized by $N$, which was the total number of data points and measured between $[0$, $\left.l_{\max }\right]$. In addition, the minimal detectable path-length $l_{\min }$ was defined as the transitional position where the signals started to increase from the base lines. The peak path-length $l_{\mathrm{p}}$ was defined as the path-length with the maximal reflectance signal. Then, the "transition distance" $\Delta l$ was calculated as $\Delta l=l_{\mathrm{p}}-l_{\min }$. The variable $\Delta l$ was calculated to help assess the path-length distribution. The noise floor was removed from the raw data during the calculation. The calculated mean path-length in tissue was then corrected by dividing a refractive index 1.37 from the path-length measured in air.

\subsection{Results}

The measurements obtained in a tendon sample are shown in Fig. 5-3. There is a reflection from the cover glass and tissue interface which was used to define the global zero point (zero path-length) in all measurements. Figure 5-3(a) shows examples of raw path-length resolved reflectance measured in tendon. At measurement angles $0^{\circ}, 22.5^{\circ}$, $45^{\circ}$ and $67.5^{\circ}$, signals with sufficient signal-to-noise were obtained at 0.0 to $0.2 \mathrm{~cm}$ away from the incident point. In general, the reflectance had smaller maximal amplitudes at smaller angles and larger distances. When measuring perpendicularly to the fibers $(\alpha=$ $90.0^{\circ}$ ), the reflectance was detectable up to $d=0.40 \mathrm{~cm}$. Figure $5-3(\mathrm{~b})$ shows the mean optical path-length as a function of measurement distance in tendon. The mean pathlength generally increased with measurement distance. When measured at $\alpha=90^{\circ}$, the mean path-length was the shortest among all other angles. The curves at angular position 
$\alpha=0.0^{\circ}, 22.5^{\circ}$, and $45.0^{\circ}$ which were similar. All curves in Fig. 5-3(b) appeared to follow quadratic or power functions.

When plotting the total reflectance (Eq. 5-1) with the mean path-length, all measurements at different distances and angles merged into one line (Fig. 5-3(c)) which can be fit using an exponential decay function with the best fit at the exponential decay constant of $9.5 \mathrm{~cm}^{-1}$ (with coefficient of determination $\mathrm{R}^{2}=0.98$ ). As shown in Fig. 5-3(d), the transition distance $\Delta l$ increased nearly linearly with the mean path-length at $\alpha=0.0^{\circ}$, $22.5^{\circ}$, and $45.0^{\circ}$. At $\alpha=67.5^{\circ}$, the transition distance increased initially until reaching a mean path-length of $\sim 0.45 \mathrm{~cm}$, and maintained a value of $\sim 0.6 \mathrm{~cm}$ thereafter. At $\alpha=$ $90.0^{\circ}$, the transition distance initially showed a similar increasing trend as at other angles, but leveled out soon at a value of $\sim 0.3 \mathrm{~cm}$ at measurement distance of $d \geq 0.05 \mathrm{~cm}$. 

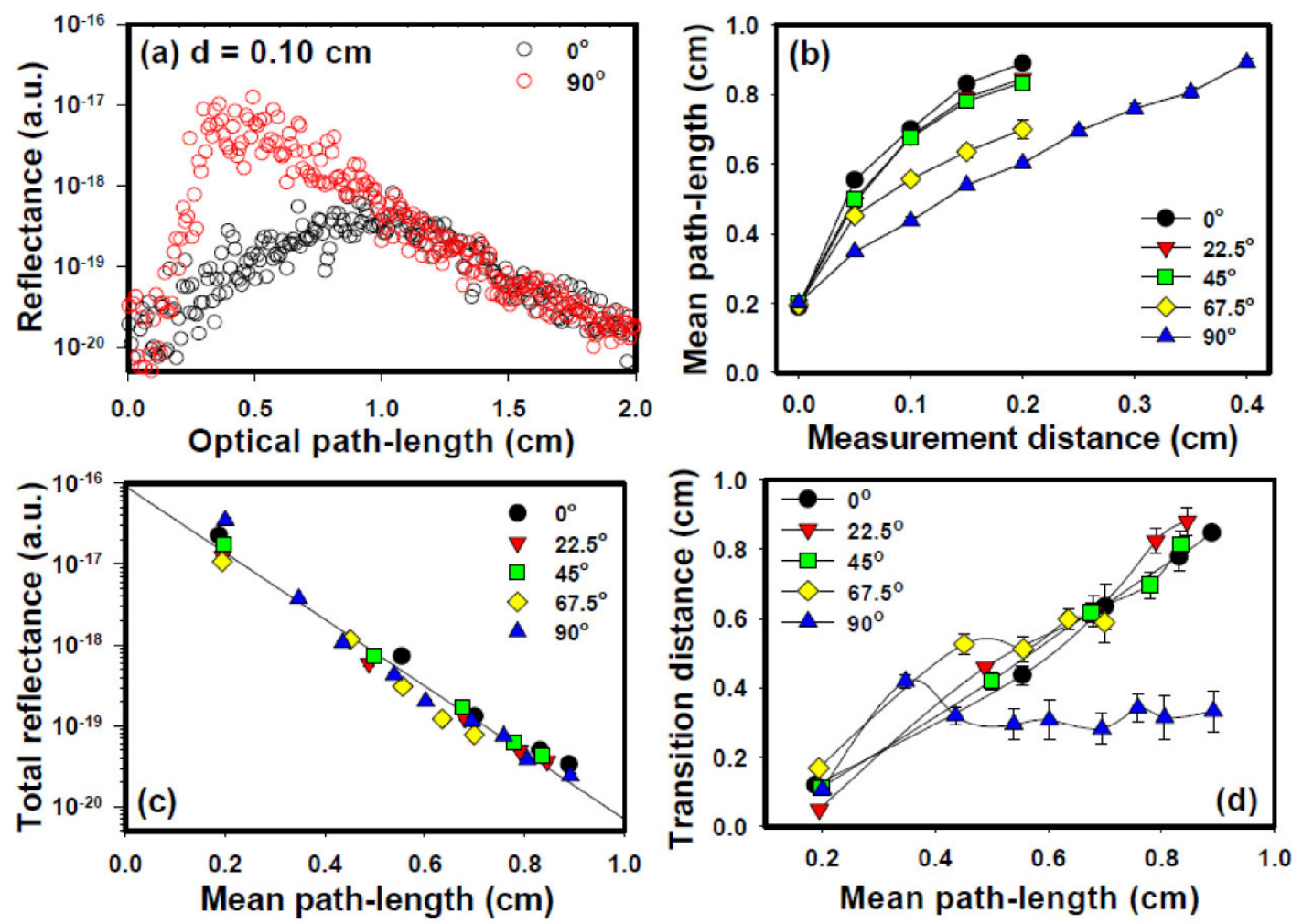

Figure 5-3: Examples of raw data measured at $0.1 \mathrm{~cm}$ away from incidence; (b) the mean pathlength as a function of measurement distance; (c) the total reflectance and (d) the transition distance as a function of the mean path-length in tendon.

Figure 5-4(a) shows examples of raw optical path-length resolved reflectance obtained in skeletal muscle. The corresponding mean path-lengths at different measurement positions were shown in Fig. 5-4(b). Similar to the results obtained in tendon, the mean path-length increased with the measurement distance. At $\alpha=90^{\circ}$, the reflectance signal was detectable at much longer measurement distances than at other angles. The curves measured at $\alpha=0.0^{\circ}, 22.5^{\circ}, 45^{\circ}$, and $67.5^{\circ}$ had similar mean pathlengths; whereas, the curve at $\alpha=90^{\circ}$ had shorter mean path-lengths than all the others. 

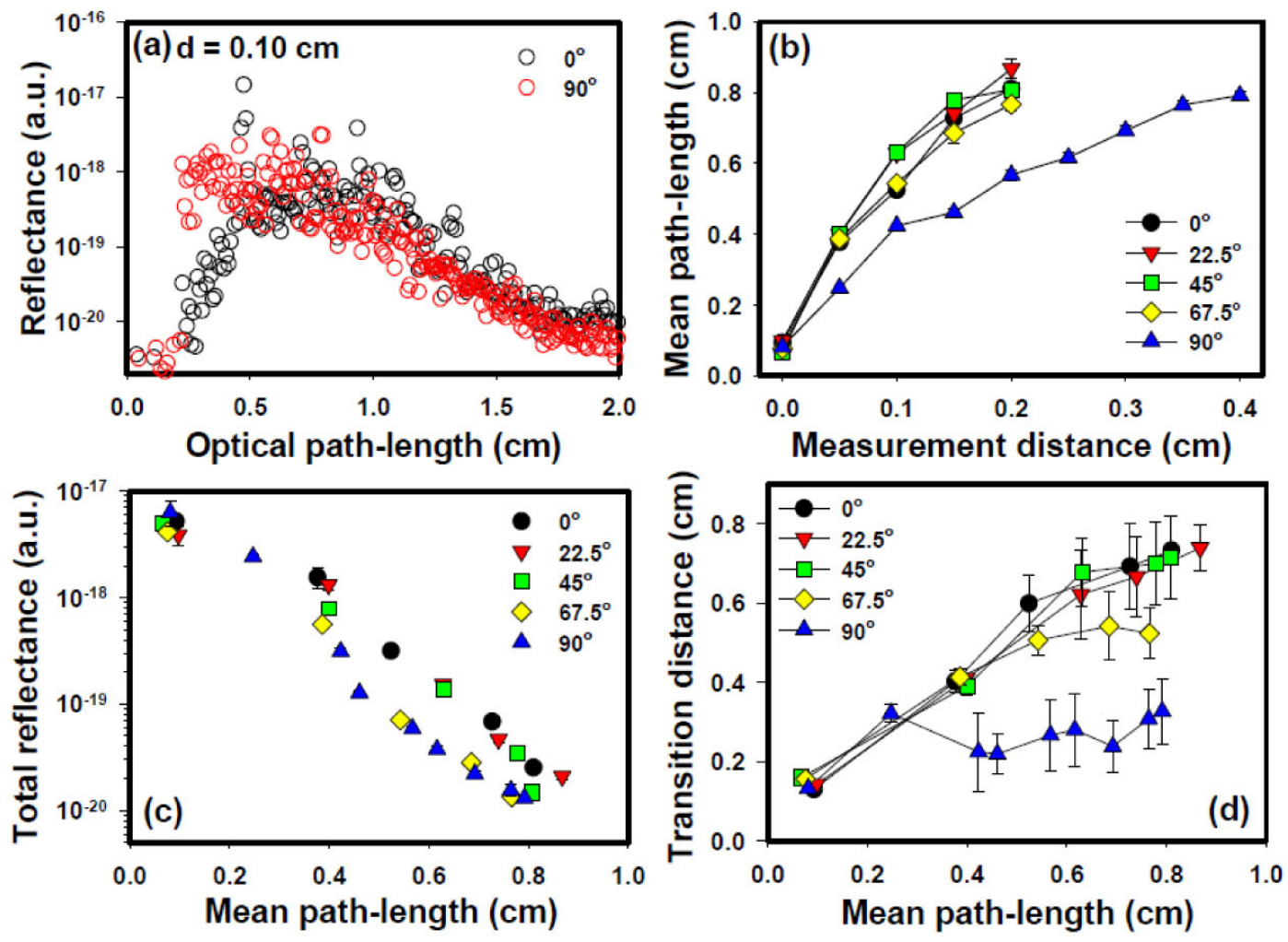

Figure 5-4: (a) Examples of raw data measured at $0.1 \mathrm{~cm}$ from incidence; (b) the mean pathlength as function of measurement distance; (c) the total reflectance and (d) the transition distance as function of the mean path-length obtained in skeletal muscle.

Figure 5-4(c) shows the total reflectance obtained in the skeletal muscle as a function of the mean optical path-length. Excluding the first a few points at the smallest measurement distance, all curves measured at different angular positions $(\alpha)$ seemed to have similar exponential decay slopes. However, at the same mean path-length, the total reflectance measured at smaller angles was higher than those measured at large angles. The transition distances as a function of the mean path-length were shown in Fig. 5-4(d). The curves shown were similar to those obtained in tendon, except that they reached a plateau at a slightly shorter mean path-length. Again, the transition distance of $\alpha=90^{\circ}$ was the smallest $(0.2 \sim 0.3 \mathrm{~cm})$ among all other angles and it stopped increasing at the shortest mean path-length of merely $d=0.05 \mathrm{~cm}$. The three curves measured at $0^{\circ}, 22.5^{\circ}$ 
and $45^{\circ}$ were similar; whereas the result at $\alpha=67.5^{\circ}$ was in between of this group and the result at $\alpha=90^{\circ}$.

\subsection{Discussion}

The raw path-length resolved reflectance clearly showed angular dependence in both tendon and muscle tissues. But it is interesting to note that the total reflectance versus mean path-length showed no anisotropic pattern in tendon (Fig. 5-3(c)). All data points measured at different angles were well fitted with one exponential curve. In other words, the reflectance measured at different angles had the same value at the same mean path-length.

To explain this observation, we examined the anisotropic diffusion theory (Johnson et al. 2002) where a diffuse tensor was applied to describe the incident angle dependent diffusion process. For fibrous samples with a predominant fiber orientation $(y$ axis in Figure 5-1), two different diffuse coefficients could be applied: $D_{y}$ represents the diffuse coefficient along the fibers, and $D$ represents that perpendicular to the fibers (along $x$ - and $z$-axis). The anisotropic diffuse equation can be written as:

$$
\frac{1}{c} \frac{\partial \Phi(r, t)}{\partial t}-\left(D \frac{\partial^{2}}{\partial x^{2}}+D_{y} \frac{\partial^{2}}{\partial y^{2}}+D \frac{\partial^{2}}{\partial z^{2}}\right) \Phi(r, t)+\mu_{a} \Phi(r, t)=Q_{0}(r, t),
$$

where $\Phi(r, t)$ is the fluence rate, $\mu_{a}$ is absorption coefficient, $c$ is the speed of light and $Q_{0}(r, t)$ is the source term. As indicated by Johnson et al. (2002), Eq.(5-3) can be converted into the conventional isotropic diffuse equation by a coordinate transformation: 
$y^{\prime}=\sqrt{D / D_{y}} y$. Thus the surface reflectance $r_{d, \alpha}(l)$ at position $(d, \alpha)$ in a semi-infinite anisotropic medium can be found from the classic isotropic diffusion solution:

$$
r_{d, \alpha}(l)=r^{i s o}\left(\sqrt{x^{2}+\left(y \sqrt{\left(D / D_{y}\right)}\right)^{2}}, l\right)
$$

where $l$ is the optical path-length, $(x, y)$ is the coordinate of the measurement point (Fig. $5-1$ ), and $r^{\text {iso }}()$ is the solution to the isotropic diffuse equation (Kienle and Patterson 1997). The reflectance depends on the measurement angle $\alpha=\tan ^{-1}(x / y)$ as defined in Fig. 5-1. Because both the total reflectance and mean path-length (Eqs. 5-1 and 5-2) become angular independent after the scaling transformation over the $y$-axis: $y^{\prime}=\sqrt{D / D_{y}} y$, the relation between the total reflectance and the mean path-length is independent of $\alpha$.

To confirm these analyses, we reanalyzed these experimental results to find whether there was a single scale factor that could transform the $y$-axis so that the mean path-length and reflectance became isotropic. Starting with the mean path-length obtained at $0^{\circ}$ and $90^{\circ}$ in Fig. 5-3(b), it was discovered that by applying a scaling factor of $\sqrt{D / D_{y}}=2.43$, the $0^{\circ}$ curve could be best fitted using the same power function obtained from the $90^{\circ}$ curve. The same transformation factor of 2.43 was then applied to all data in Fig. 5-3(b) \& (c). The results were shown in Figure 5-5 as a function of the corrected distance $\left(x^{2}+2.43^{2} y^{2}\right)^{1 / 2}$. It is clear that the angular dependency disappeared in both graphs as predicted from the anisotropic diffuse theory. Figure 5-5 confirmed that the total reflectance (Eq. 5-1) vs. mean path-length curve was independent of measurement angle $\alpha$ in tendon tissue as shown in Figure 5-3(c). 

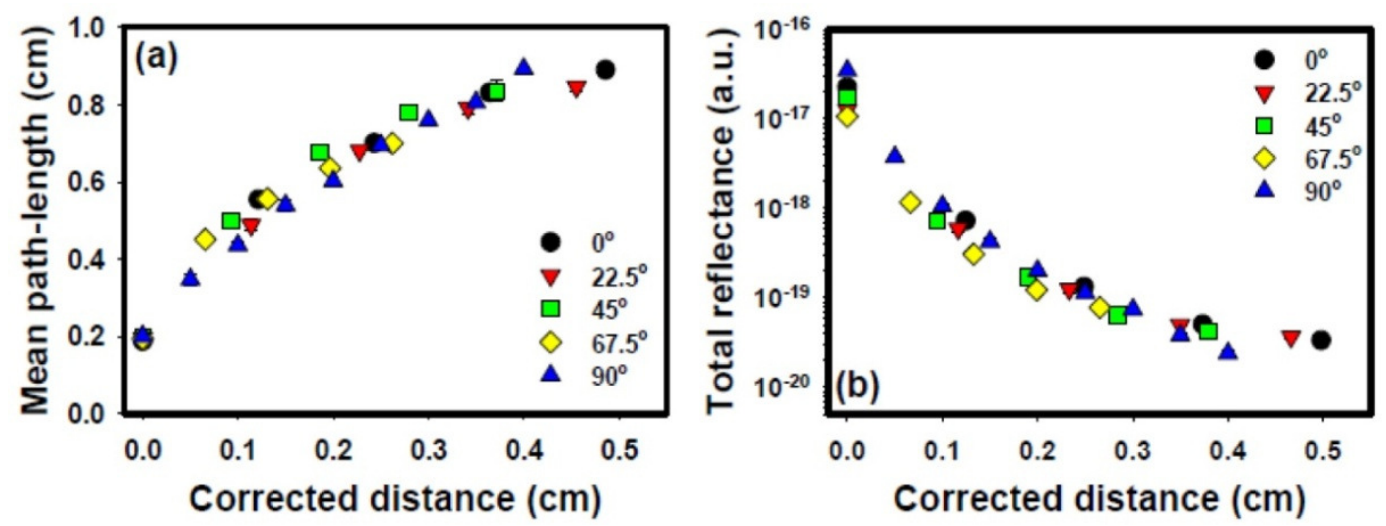

Figure 5-5: The mean path-length (a) and total reflectance (b) after transforming the y-axis by $y^{\prime}=2.43 y$ for the same tendon data shown in Fig. 5-2(b) \& (c), respectively.

The muscle samples showed quite different reflectance vs. mean path-length curves (Fig. 5-3(c)). Despite the fact that all curves in Fig. 5-3(c) followed a similar decaying trend, they had different y-axis offset values. The transformation procedure mentioned above failed to eliminate the angular dependency in both mean path-length and total reflectance as shown in Fig. 5-5. Although the mean path-lengths at $0^{\circ}$ and $90^{\circ}$ were matched by using the obtained transformation factor $\sqrt{D / D_{y}}=2.03$, the corresponding reflectance curves were not matched using the same scaling factor (Fig. 5$5(\mathrm{~b}))$.
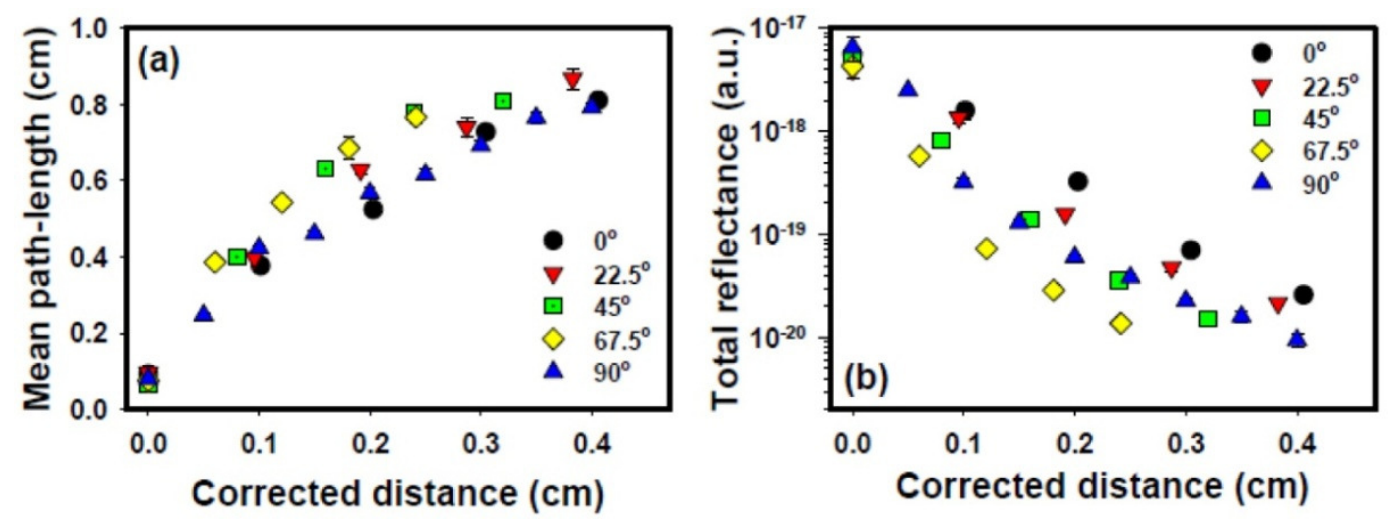

Figure 5-6: The mean path-length (a) and total reflectance (b) after transforming the y-axis by $y^{\prime}=2.03 y$ for the same muscle data shown in Fig. 5-3(b) \& (c), respectively. 
A closer examination of Figs. 5-3 and 5-4 revealed some interesting differences between the results obtained in tendon and muscle. In tendon, the total reflectance measured at a position along $0^{\circ}$ was equal to that measured at a larger distance along $90^{\circ}$ (Fig. 5-3(c) and Fig. 5-3(b)). In other words, the equi-intensity profiles were elongated toward $90^{\circ}$, exactly as that predicted from fibrous scatterings (Nickell et al. 2000; Shuaib and Yao 2010) at small detection distances. However, Figure 5-4(b) and (c) suggested that similar total reflectance was obtained at roughly the same detection distance along $0^{\circ}$ and $90^{\circ}$ in muscle. In other words, the equi-intensity profiles in muscle were more symmetric at $0^{\circ}$ and $90^{\circ}$ than that in tendon within the small measurement distances of $\sim 4$ $\mathrm{mm}$. It is expected that photons were still scattered toward $90^{\circ}$ by the cylindrical shaped muscle fibers. Thus the more symmetric equi-intensity patterns implied the existence of other mechanisms that scattered photons toward $0^{\circ}$ in muscle. As revealed in previous studies (Ranasinghesagara and Yao 2007, 2008), sarcomere diffraction clearly fits this profile. It is worth mentioning that skeletal muscle and fibrous tissues also had different equi-intensity reflectance profiles at measurement distances much larger than those used in this study (Nickell et al. 2000; Ranasinghesagara and Yao 2007). When measured far away from the incidence, the equi-intensity profiles in fibrous samples form an ellipse that is stretched along the fibers $\left(0^{\circ}\right)$ because of the smaller scattering coefficient along the fibers (Shuaib and Yao 2010). Such elliptical patterns (elongated along fibers) were not observed in muscle. On the contrary, the patterns in some types of muscles (Ranasinghesagara et al. 2010) tend to elongate perpendicularly to the fibers at larger distance. 
The transition distance also had strong angular dependency in both tendon and muscle as shown in Fig. 5-2(d) and 5-3(d). It was interesting that the transition distance was much smaller and did not change much with mean path-length in both tendon and muscle when measured at $90^{\circ}$, i.e. the reflectance reached maximal values at smaller path-lengths at $90^{\circ}$. Because of the smaller mean path-length, the reflectance decayed slower with detection distance and can be detected at much larger distance at $90^{\circ}$ as shown in Figs. 5-2 and 5-3. However, the same aforementioned $y$-axis transformation didn't eliminate the angular dependency in transition distance in both tendon and muscle. Since the transition distance was primarily determined by photons experiencing few number of scattering events, the diffuse equation may not be applicable to them.

The aforementioned results were replicated in three more tendon samples and six more muscle samples, all of which were from different animals. Specifically, angular dependency in reflectance was eliminated in all tendon samples (shown in Fig. 5-7 to Fig. 5-9) when representing reflectance as a function of mean path-length (as illustrated in Fig. 5-2(c)). However, the same phenomenon was not observed in any of the six muscle samples measured in this study (shown in Fig. 5-10 to Fig. 5-15). It should be pointed out that reflectance was measured by using a probe positioned at $60^{\circ}$ from the sample surface (or $30^{\circ}$ from the normal). It has been shown that surface reflectance has similar angular distributions in both isotropic and anisotropic tissues (Xia and Yao 2007a). Therefore, it is likely that similar results will be obtained by using other detection angles. 

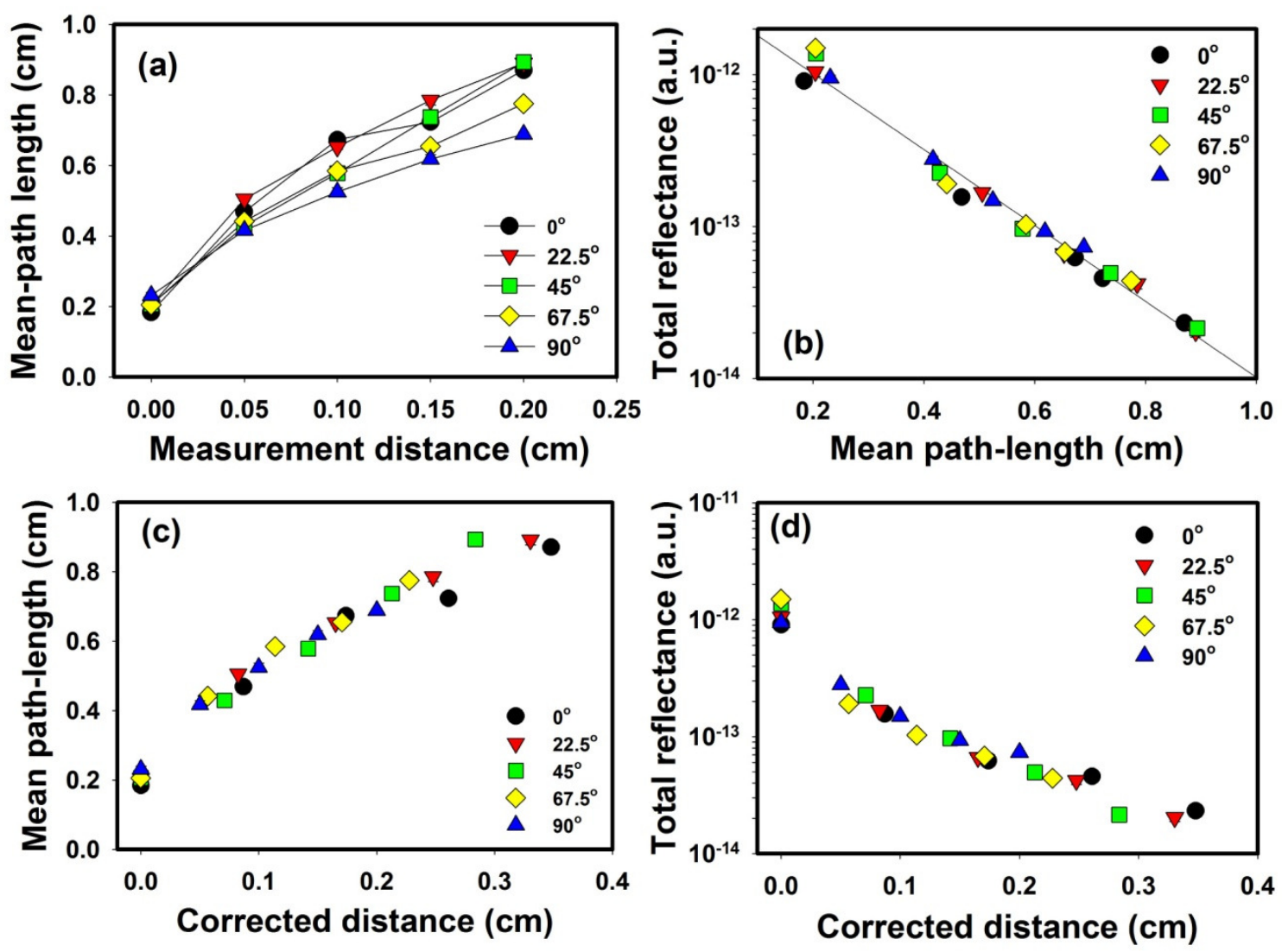

Figure 5-7: (a) The mean path-length and (b) total reflectance as function of measurement distance obtained in tendon. Also note the mean path-length (c) and total reflectance (d) after transforming the $y$-axis by $y^{\prime}=1.71 y$. 

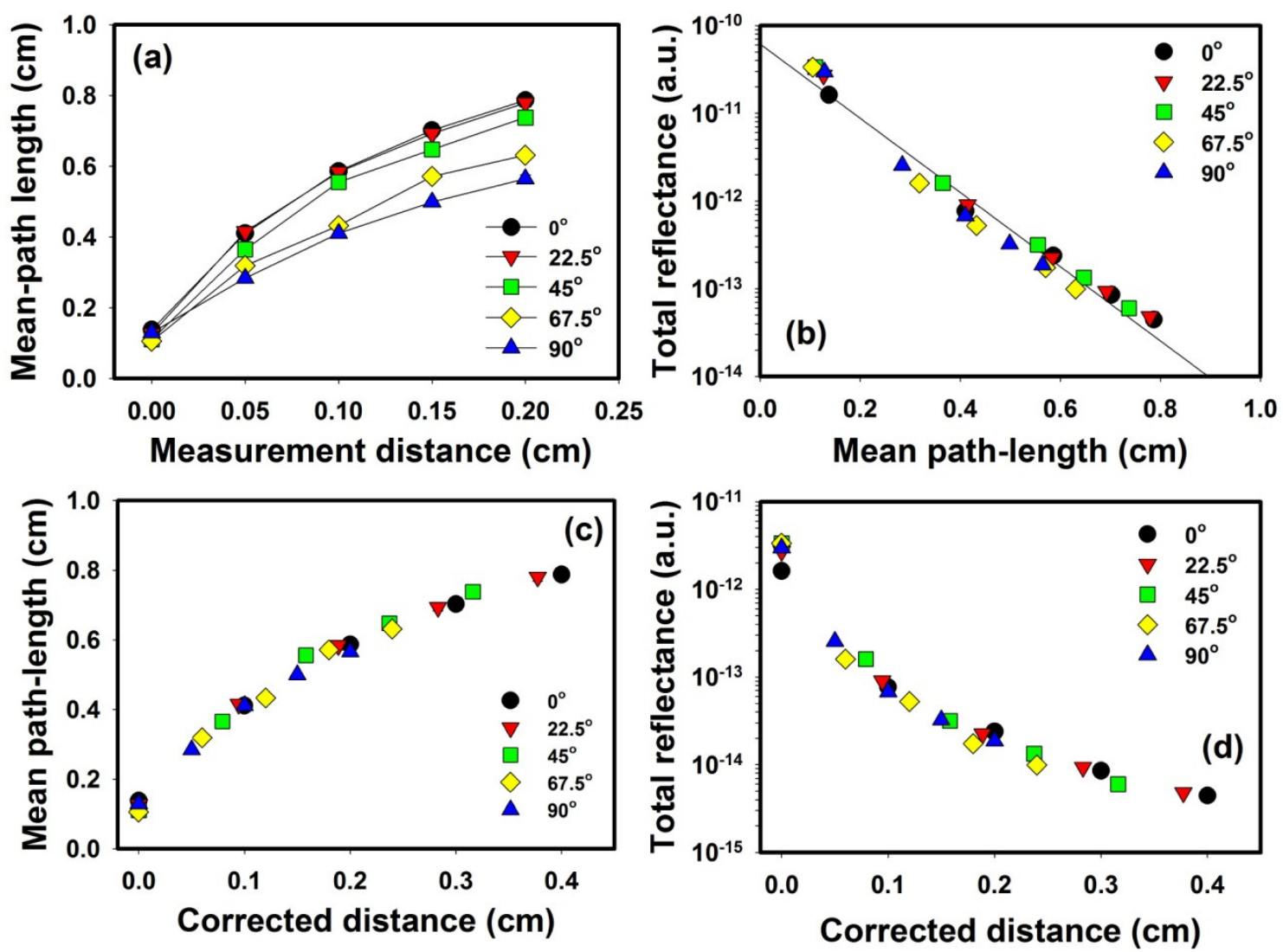

Figure 5-8: (a) The mean path-length and (b) total reflectance as function of measurement distance obtained in tendon. Also note the mean path-length (c) and total reflectance (d) after transforming the $y$-axis by $y^{\prime}=2.00 y$. 

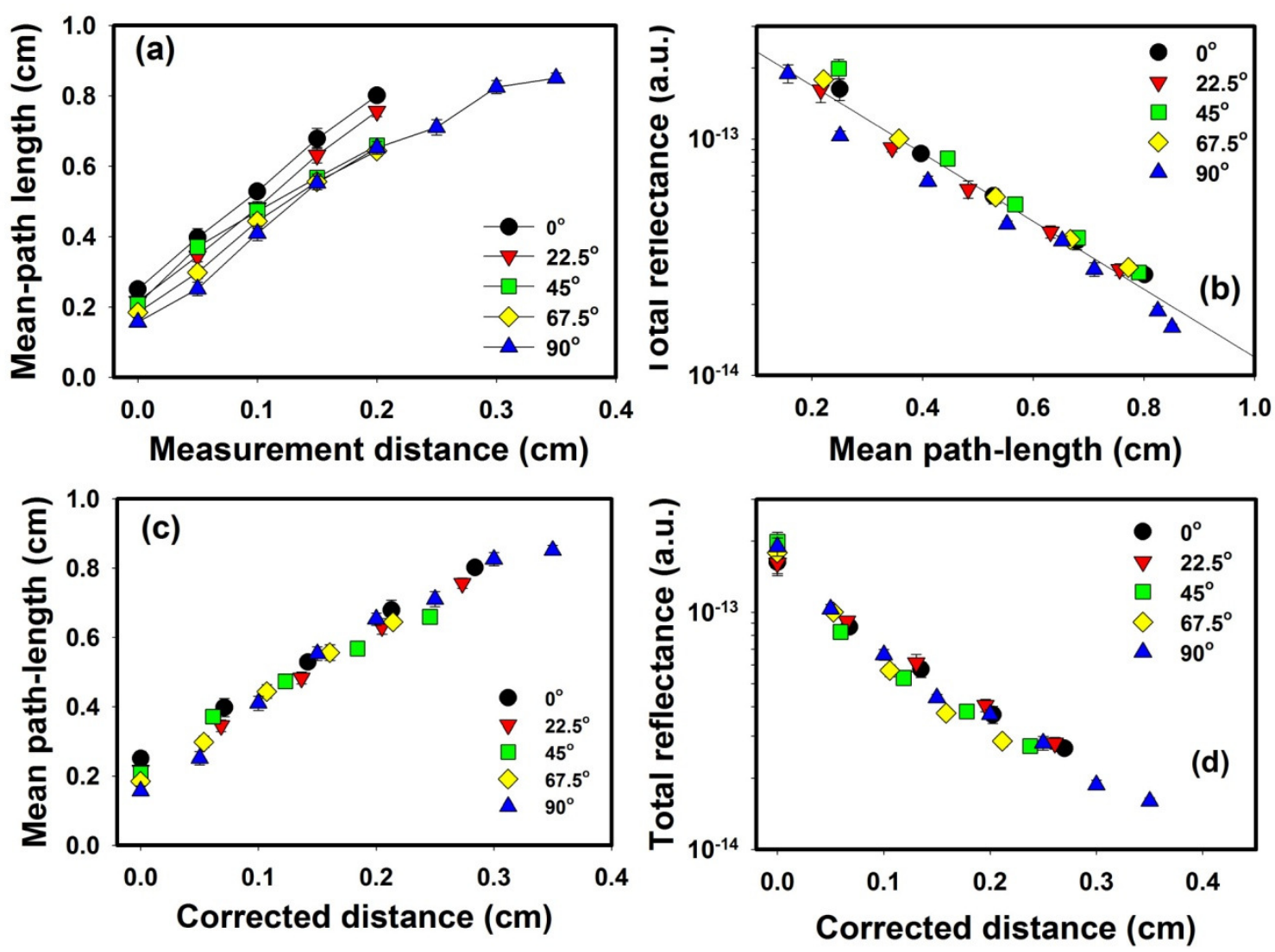

Figure 5-9: (a) The mean path-length and (b) total reflectance as function of measurement distance obtained in tendon. Also note the mean path-length (c) and total reflectance (d) after transforming the $y$-axis by $y^{\prime}=1.42 y$. 

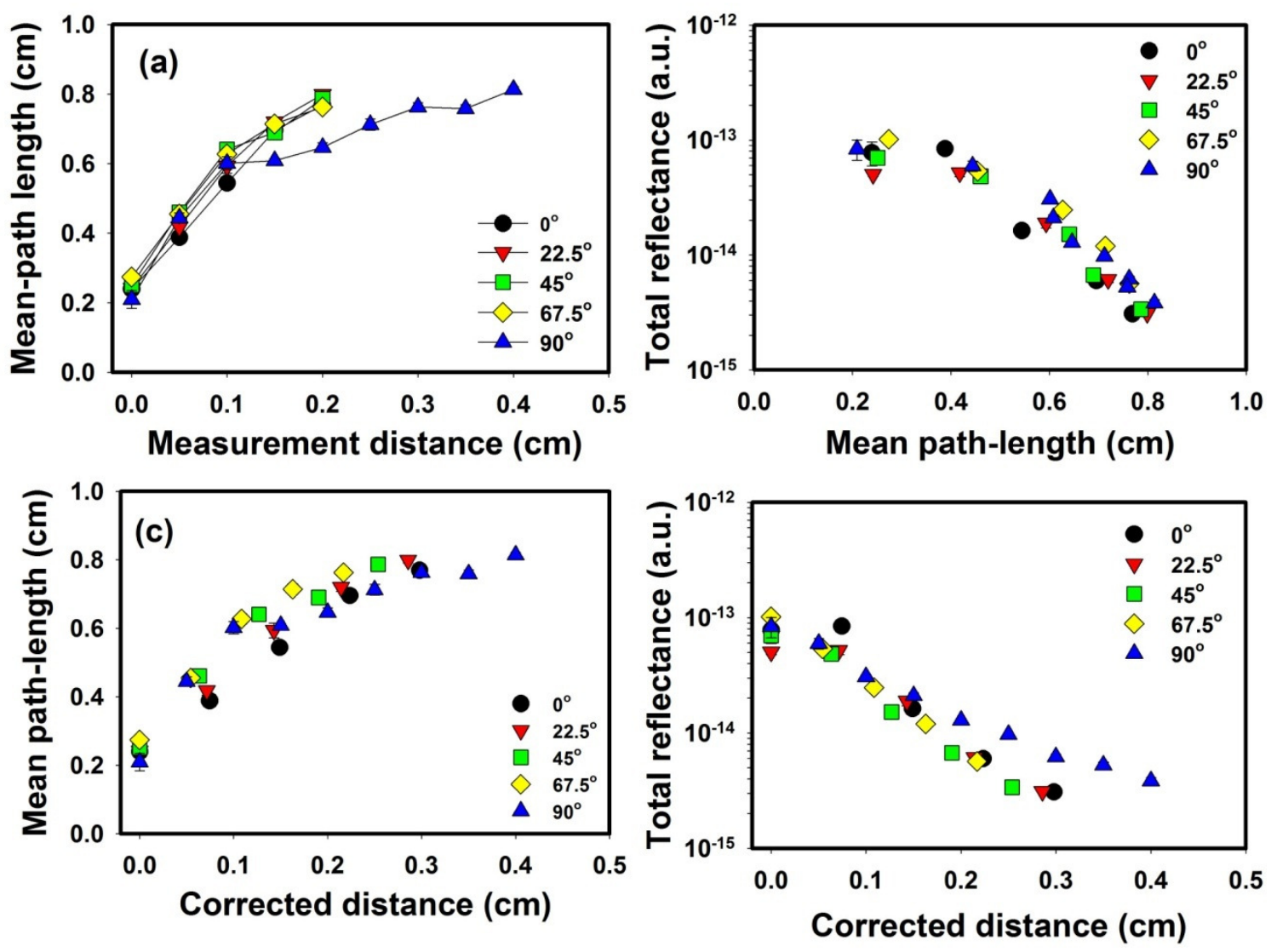

Figure 5-10: (a) The mean path-length and (b) total reflectance as function of measurement distance obtained in skeletal muscle. Also note the mean path-length (c) and total reflectance (d) after transforming the $y$-axis by $y^{\prime}=2.43 y$. 

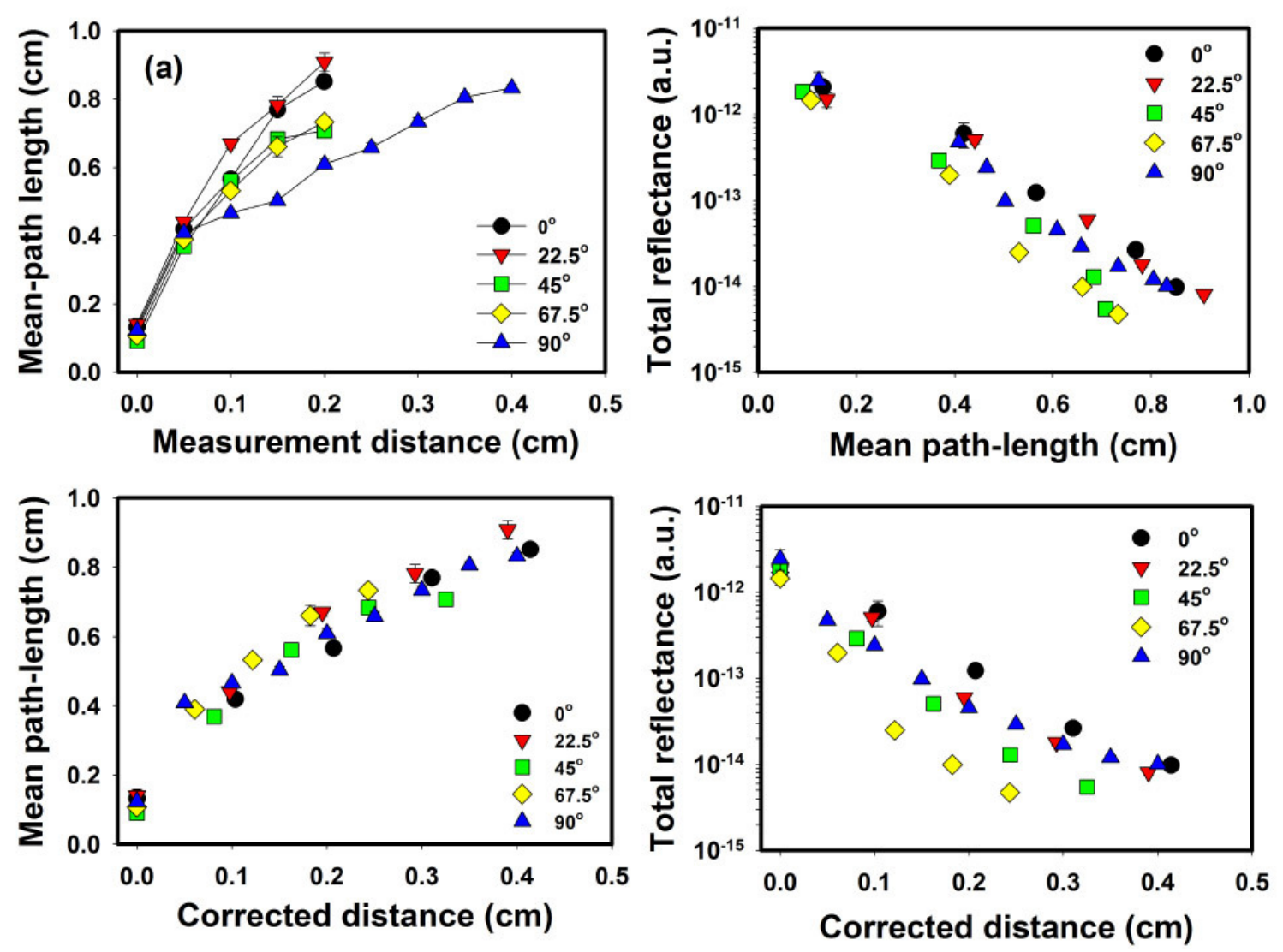

Figure 5-11: (a) The mean path-length and (b) total reflectance as function of measurement distance obtained in skeletal muscle. Also note the mean path-length (c) and total reflectance (d) after transforming the $y$-axis by y'=2.43y. 

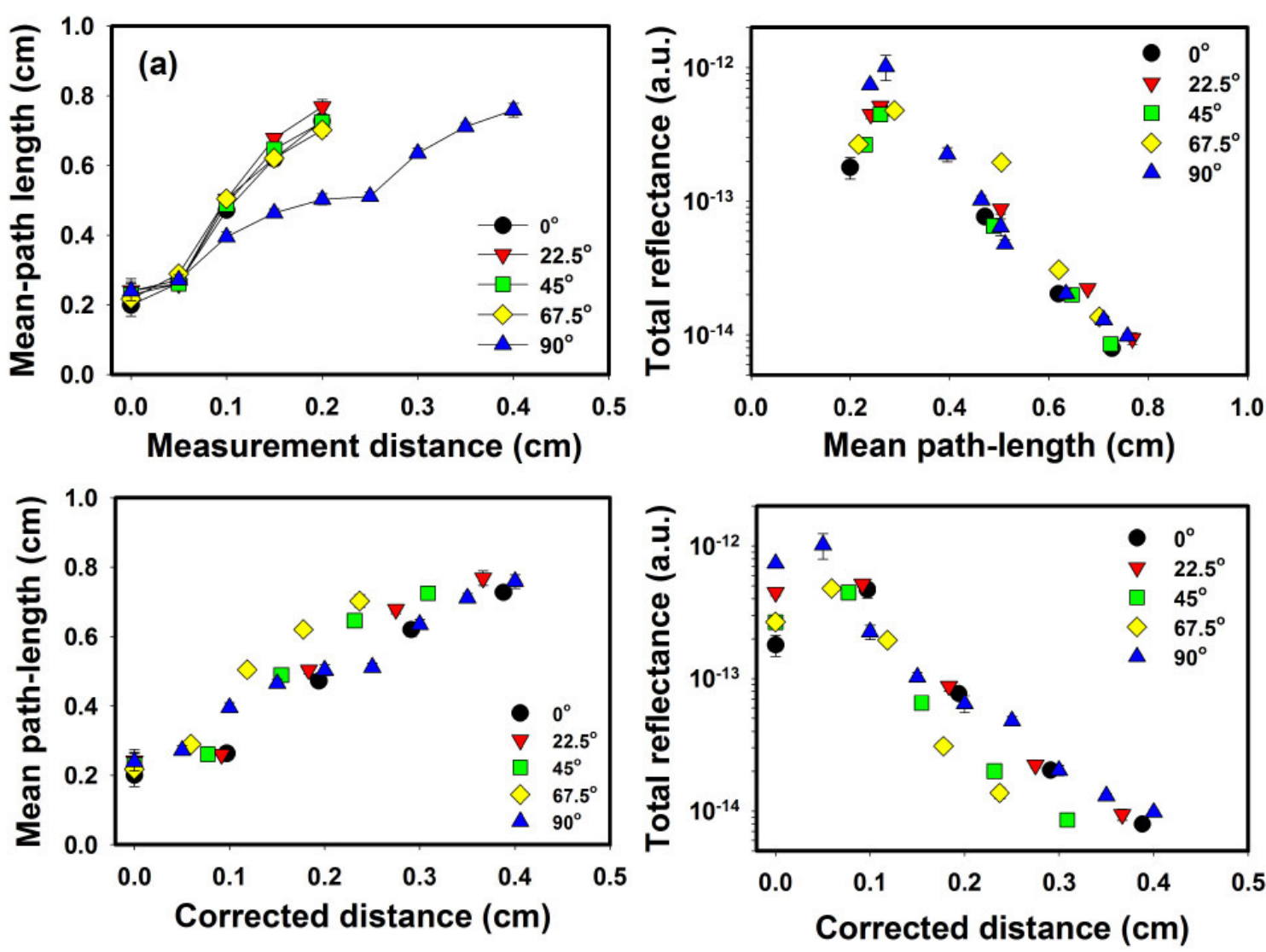

Figure 5-12: (a) The mean path-length and (b) total reflectance as function of measurement distance obtained in skeletal muscle. Also note the mean path-length (c) and total reflectance (d) after transforming the $y$-axis by $y^{\prime}=1.94 y$. 

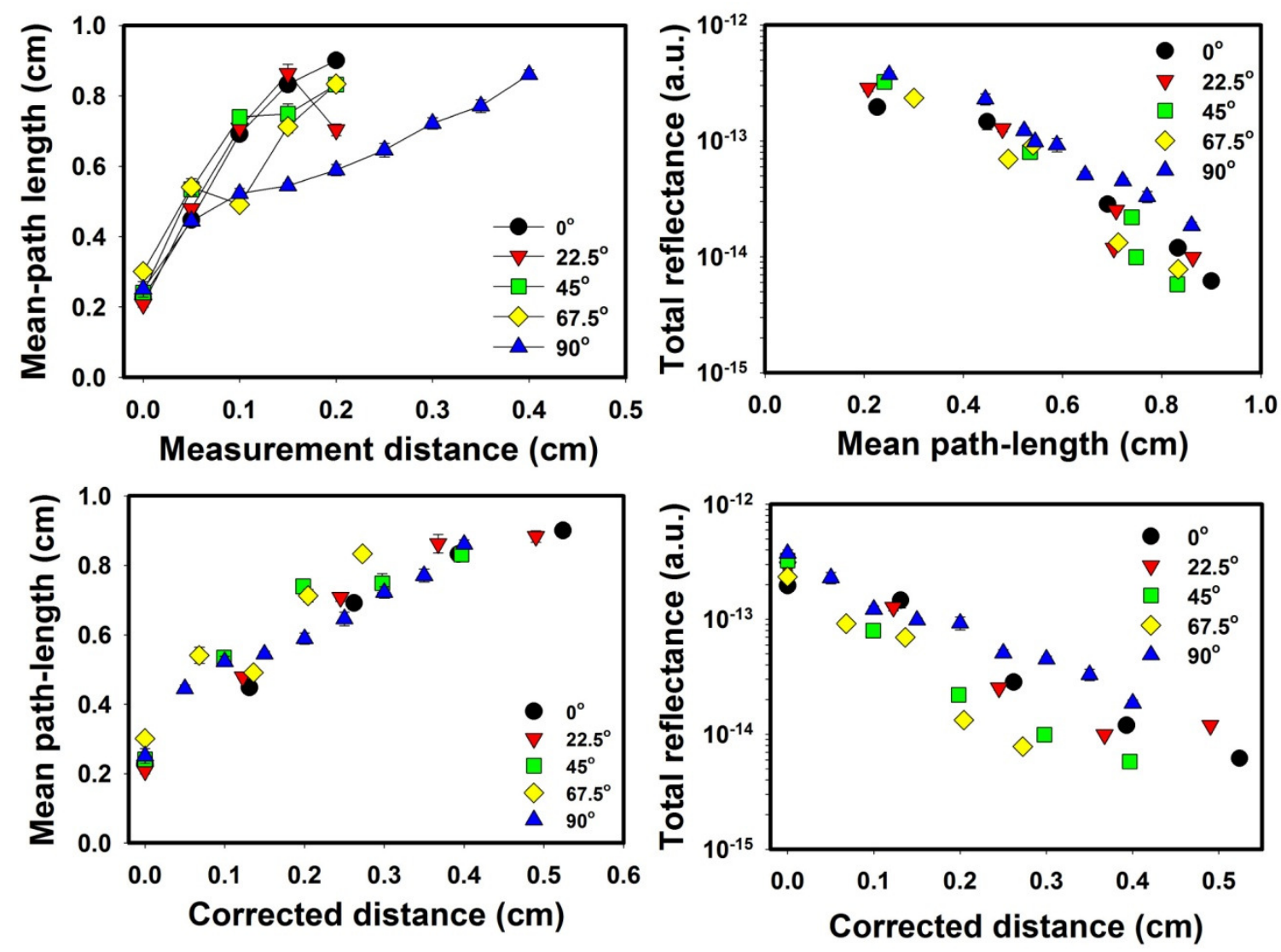

Figure 5-13: (a) The mean path-length and (b) total reflectance as function of measurement distance obtained in skeletal muscle. Also note the mean path-length (c) and total reflectance (d) after transforming the $y$-axis by $y^{\prime}=2.62 y$. 

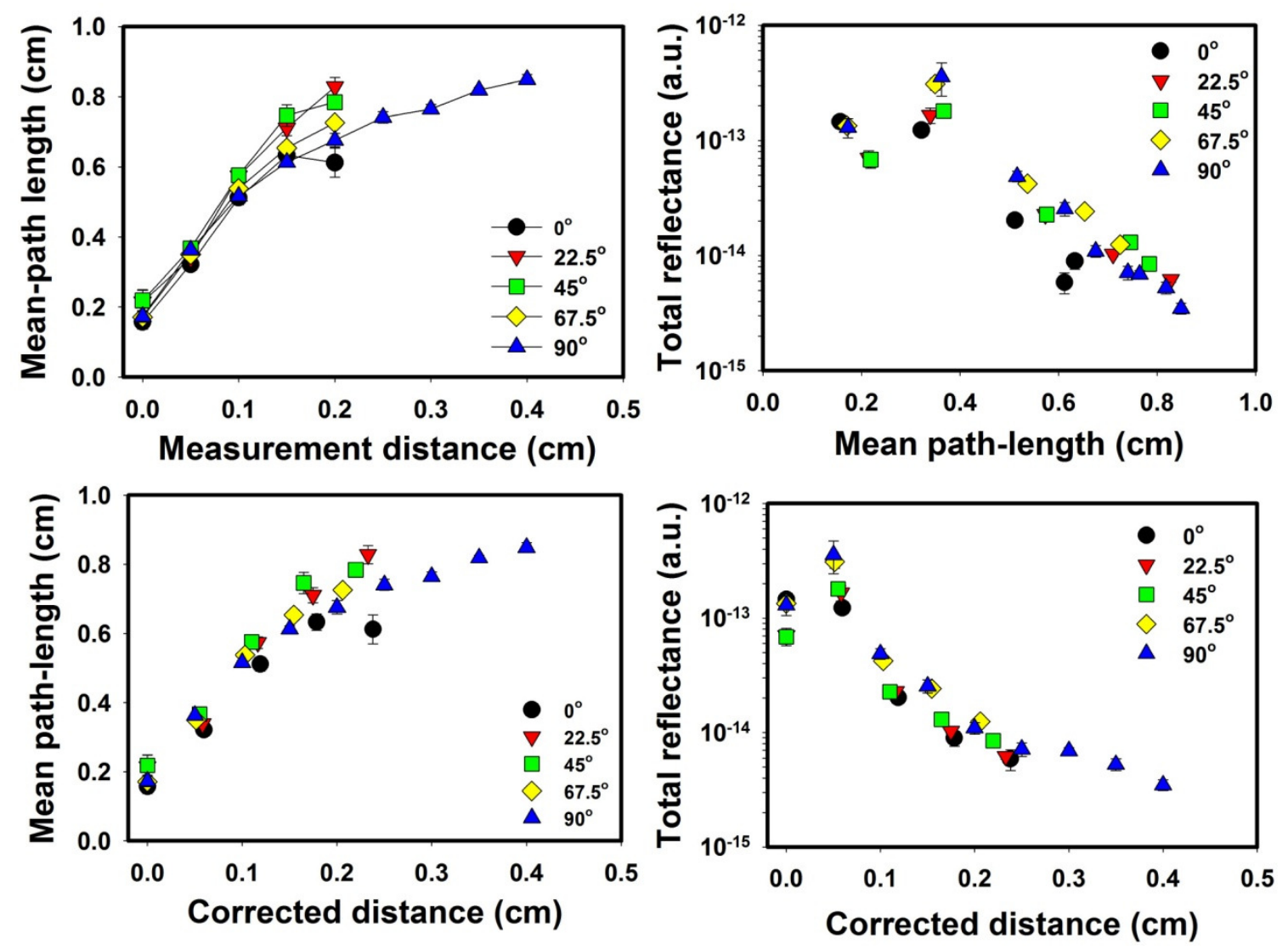

Figure 5-14: (a) The mean path-length and (b) total reflectance as function of measurement distance obtained in skeletal muscle. Also note the mean path-length (c) and total reflectance (d) after transforming the $y$-axis by $y^{\prime}=1.19 y$. 

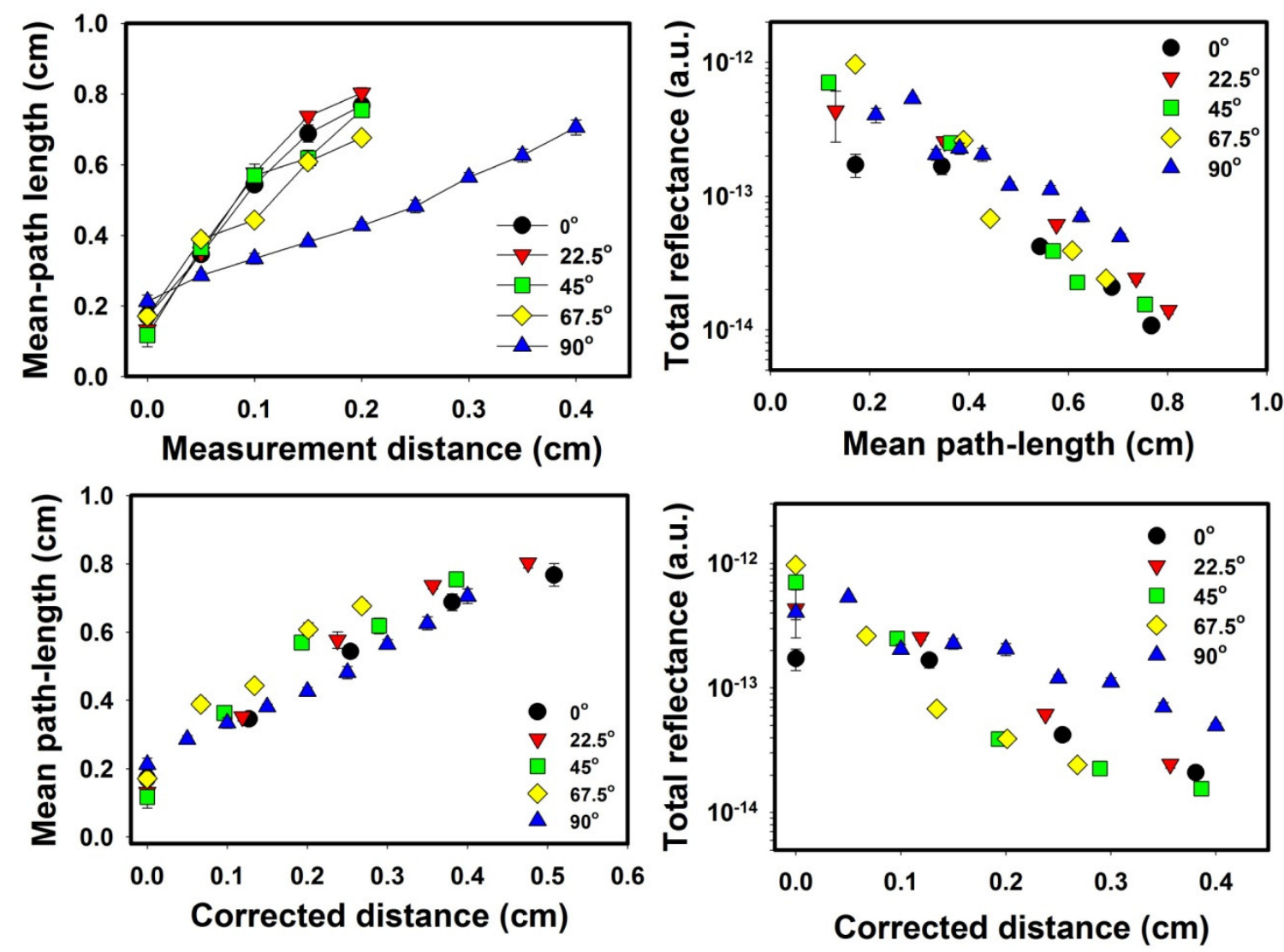

Figure 5-15: (a) The mean path-length and (b) total reflectance as function of measurement distance obtained in skeletal muscle. Also note the mean path-length (c) and total reflectance (d) after transforming the $y$-axis by $y^{\prime}=2.54 y$.

\subsection{Conclusion}

In summary, a Mach-Zehnder low coherent interferometer was used to study the light propagation in tendon and skeletal muscle tissues. The raw path-length resolved reflectance showed strong angular dependency in both tissues. Quantitative analyses revealed both similarities and significant differences in tendon tissue and skeletal muscles. When reflectance was represented as a function of mean path-length, the angular dependency was eliminated in tendon but not in muscle. Experimental 
observations in tendon sample corroborated the existing knowledge on light scattering in fibrous materials. These results suggested that an anisotropic diffuse model may be applied to measure optical properties in fibrous tissues such as tendon. However, it cannot satisfactorily explain optical reflectance measured in skeletal muscles. Additional scattering mechanisms such as sarcomere effect need to be considered in order to fully describe photon migration in muscle. On the other hand, if the periodic sarcomere structures influence light propagation in muscle, optical techniques may be developed to detect some sarcomere related muscle diseases 


\section{CHAPTER 6}

\section{MEASURING TENDON OPTICAL PROPERTEIS BY USING PATH-LENGTH RESOLVED REFLECTANCE}

\subsection{Introduction}

Optical methods are appealing as diagnostic means in medicine because they are non-invasive, relatively low in cost, and can provide functional information. Optical diagnosis relies on the different optical properties of pathologic versus healthy tissue or on physiologic information that can be derived from the assessment of the optical properties. Diffusion approximation (Farrell et al. 1992) of the radiative transport theory is usually applied to study light propagation in highly scattering tissue. In many cases, biological tissues are generally assumed to be isotropic turbid medium where light scattering is independent of the incident direction. However, this assumption becomes invalid in anisotropic tissues such as tendon (Kienle et al. 2004), dentin (Kienle et al. 2003), skin (Nickell et al. 2000) and skeletal muscle (Ranasinghesagara and Yao 2007). Since anisotropic tissues are abundant in human and animals, a thorough study on light propagation in such tissues is critical for correctly interpreting optical measurements obtained in them.

Time or path-length resolved optical measurement can be applied to measure absorption and reduced scattering coefficient of the turbid medium (Delpy et al. 1988). The diffuse model was shown to able to describe the optical reflectance in scattering samples that is measured using the Michelson-based interferometric (Popescu and Dogariu 1999). Recently, Fan et al. (2011) applied Mach-Zehnder based interferometric 
to study light propagation and showed the reflectance in tendon is angular dependent, but such anisotropy in reflectance was eliminated when representing the reflectance as a function of mean path-length.

In this study, we used a single-mode fiber optic Mach-Zehnder based LCI to measure optical properties in tendon by fitting time-resolved reflectance using diffuse theory.

\subsection{Materials and Methods}

A single mode fiber optic Mach-Zehnder based LCI (Fig. 5-1), described in detail in Chapter 5, was used to measure the path-length resolved measurement for the different tissues. To validate the Mach-Zehnder low coherence interferometer measurements, two sets of samples were prepared. First, isotropic scattering phantoms were made by diluting $20 \%$ Intralipid solution (Fresenius Kabi, Uppsala, Sweden) into 15\%, 10\%, and 8\% volume concentrations. The calculated reduced scattering coefficients for the various $(20 \%, 15 \%, 10 \%$, and $8 \%)$ intralipid concentrations from research by Flock et al. (1992) at $1287 \mathrm{~nm}$ are $\mu^{\prime}{ }_{s}=34.5,25.9,17.5$, and $13.8 \mathrm{~cm}^{-1}$, respectively. The theoretical water absorption coefficient established by Kou et al. (1993) is $1.17 \mathrm{~cm}^{-1}$ at $\lambda_{o}=1287 \mathrm{~nm}$. Secondly, $15 \%$ concentration of intralipid solutions with three different India ink concentrations $(0 \%, 1 \%$, and 2\%) (Salis Int'l. Inc., Golden, CO) were made. The absorption coefficient India ink concentrations of $1 \%$ and $2 \%$ were interpreted from previous study by Xia and Yao (2007b) to be $\mu_{a} \approx 0.22$ and $0.37 \mathrm{~cm}^{-1}$, respectively. To be noted, the sample dimension of the sample is much larger than the transport mean free path. In other words, the sample can be considered as a semi-infinite medium. 
In addition, chicken liver was used as an isotropic sample. Afterward, a fresh bovine tendon sample was obtained from the meat science laboratory at the University of Missouri-Columbia. All surface fat on sample surface was carefully removed before measurements.

The analytical solution of the diffusion equation in an isotropic semi-infinite medium using extrapolated boundary condition (Hielscher et al. 1995; Kienle and Patterson 1997) was used to derive the optical reduced scattering $\left(\mu_{s}^{\prime}\right)$ and absorption $\left(\mu_{a}\right)$ coefficients from the path-length resolved reflectance:

$$
R(\rho, l)=M \frac{c e^{-\mu_{a} l} e^{-\frac{\rho^{2}}{4 D l}}}{2(4 \pi D)^{3 / 2}(l)^{5 / 2}}\left[z_{o} e^{\frac{-z_{o}^{2}}{4 D l}}+\left(z_{o}+2 z_{b}\right) e^{\frac{-\left(z_{o}+2 z_{b}\right)^{2}}{4 D l}}\right]
$$

where $c$ is the speed of light in tissue, $l$ is the optical path-length, and $\rho$ is the distance between the measurement and the incident; $M$ is the multiplicative factor; $D$ is the optical diffusion constant: $D=1 / 3\left(\mu^{\prime}{ }_{s}+\mu_{a}\right)$ and $z_{b}=2 D(1+\operatorname{Reff}) /(1-\operatorname{Reff})$, where Reff is the fraction of optical reflection at the boundary as described in (Haskell et al. 1994). $z_{o}=3 D$ is the transport mean free path. In the calculation, the refractive of indices $n$ were: 1.33 for the Intralipid solution, 1.37 for biological tissue such as liver, tendon, and 1.0 for the external medium (air). The optical path-length was divided by the refractive index of the medium to obtain the correct optical path-length. The Levenberg-Marquardt nonlinear fitting in MATLAB (The Mathworks Inc., Natick, MA) with global optimization tool was applied to fit Eq. (6-1) to the experimental measurements and derive three parameters: 1) the reduced scattering coefficient $\left.\mu_{s}^{\prime}, 2\right)$ the absorption coefficient $\mu_{a}$, and 3) the multiplicative factor $M$. For the uncertainty in the fitting, the confidence intervals 
calculated using MATLAB utilized the derived parameters: coefficient estimates, residuals, and the Jacobian matrix from the nonlinear fitting algorithm.

To ensure the validity of the diffuse approximation, only the decaying portion of the path-length resolved reflectance profile was used in the fitting. Specifically, the fitting started after the optical path-length was larger than $0.3 \mathrm{~cm}$ (the corresponding time is $0.01 n s$. A median filter was used to reduce the noise in the path-length resolved reflectance; and thereby achieve better diffuse fitting (Eq. 6-1).

\subsection{Results}

The example raw optical path-length resolved reflectance measured in $20 \%$ intralipid solution is shown in Fig. 6-1. The measurement distance was from 0.0 to 0.2 $\mathrm{cm}$ with a step size of $0.05 \mathrm{~cm}$.

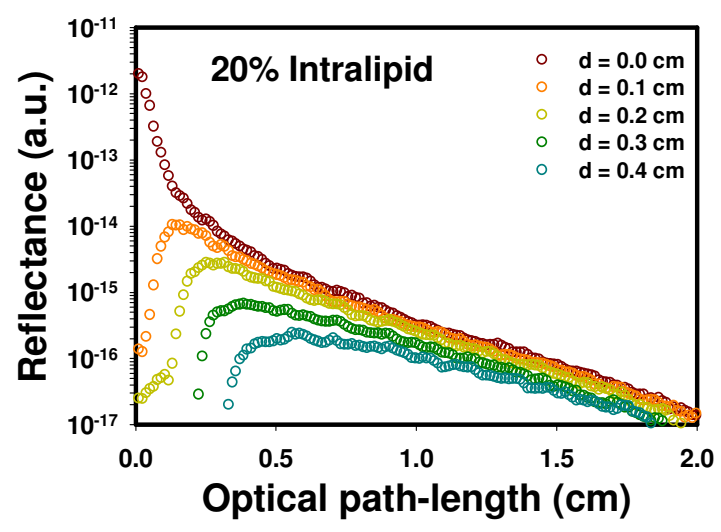

Figure 6-1: Example raw optical path-length resolved reflectance obtained in intralipid (20\%).

Due to the isotropic characteristic of the intralipid solution, the measurements acquired at different angular positions $(\alpha)$ were identical. To be noted, the zero point, 
otherwise known as the zero optical path-length, was set by using the light reflected back from the cover glass. It can be seen that the reflectance increased quickly to a maximum and decreased slowly thereafter. At larger measurement distances, the entire reflectance profiles were lower in magnitude due to higher attenuation. In addition, the maximal reflectance (or the reflectance peak) increased with measurement distance.

Figure 6-2(a) shows sample fitting results using Eq. (6-1) at $d=0.0,0.1$, and 0.2 $\mathrm{cm}$ in the $20 \%$ Intralipid solution. To ensure the validity of the diffuse approximation, only the decaying portion of the profile was used in the fitting. The determined reduced scattering $\mu_{s}^{\prime}$ and absorption $\mu_{a}$ coefficients are shown in Fig. 6-2(b). The average reduced scattering coefficient is $\sim 33 \mathrm{~cm}^{-1}$ for $d=0.1 \mathrm{~cm}$. This value is very close to that calculated from published data (Flock et al. 1992) $\mu_{s}^{\prime}=35 \mathrm{~cm}^{-1}$. The absorption coefficient was slightly higher at smaller distances, but stabilized at larger distances $(d>$ $0.5 \mathrm{~cm}$ ). The average absorption coefficient is $\mu_{a} \approx 1.15 \mathrm{~cm}^{-1}$ for $d=0.15 \mathrm{~cm}$, which is close to the theoretical water absorption of $1.17 \mathrm{~cm}^{-1}$ at $\lambda_{o}=1287 \mathrm{~nm}$ (Kou et al. 1993).
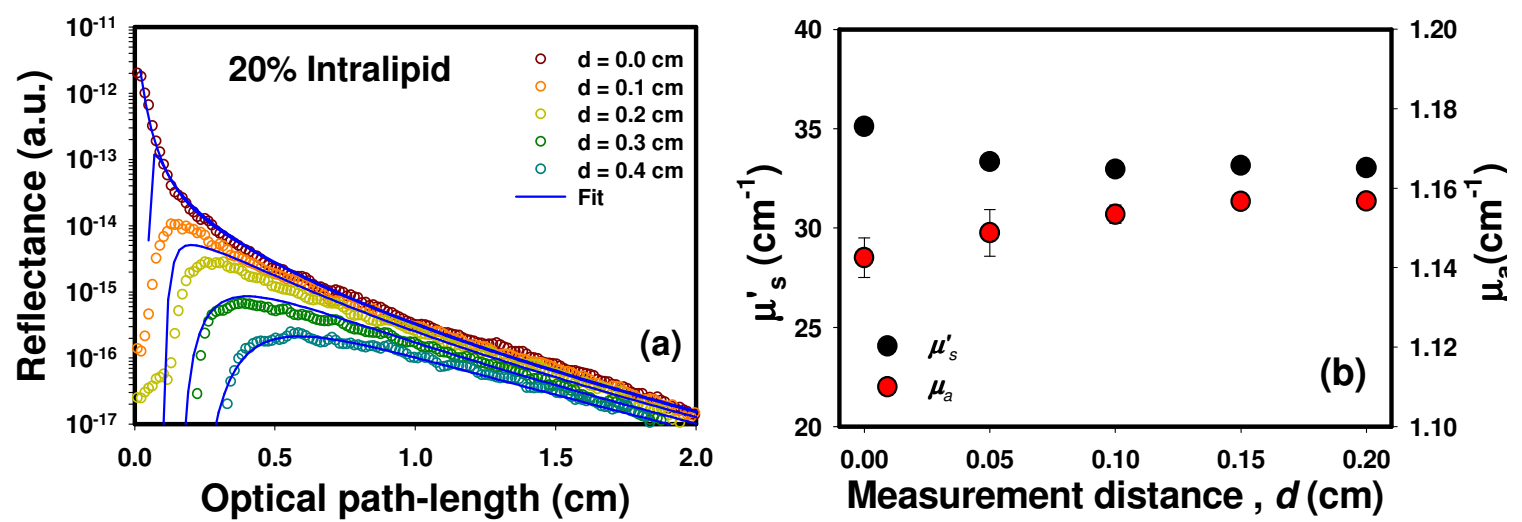

Figure 6-2: (a) Sample fitting results for $20 \%$ intralipid optical path-length measured (circles) at distance 0.0, 0.05, 0.1, 0.15, and $0.2 \mathrm{~cm}$ using Eq. (6-1). (b) The determined reduced scattering $\mu_{s}^{\prime}$ and absorption $\mu_{a}$ coefficients for measurement at distance from 0.0 to $0.2 \mathrm{~cm}$. The detection angle $\phi$ is $60^{\circ}$. Error bars represent the $95 \%$ c.i. of the fitted values. 
Figure 6-3(a-b) shows the reduced scattering and absorption coefficients for different intralipid concentrations solutions at different measurement distances. It can be seen that the determined reduced scattering coefficient $\mu_{s}^{\prime}$ is stable at large distance. The determined absorption coefficients $\mu_{a}$ are not consistent at small distance measurement $(\mathrm{d}<0.05 \mathrm{~cm})$; however, as the distance increases the derived $\mu_{a}\left(\approx 1.15 \mathrm{~cm}^{-1}\right)$ becomes close to the theoretical value. Figure 6-3(c) shows the reduced scattering and absorption coefficient for different intralipid concentration solutions at measurement distance $d=$ $0.10 \mathrm{~cm}$. The reduced scattering coefficient $\mu_{s}$, has a linear property with a slope of 0.94 $\left(\mathrm{R}^{2}=0.992\right)$. Whereas for the absorption coefficient, increasing the intralipid concentration has no significant effect on the determined absorption coefficient (the slope is $\approx 0.0$ ). 

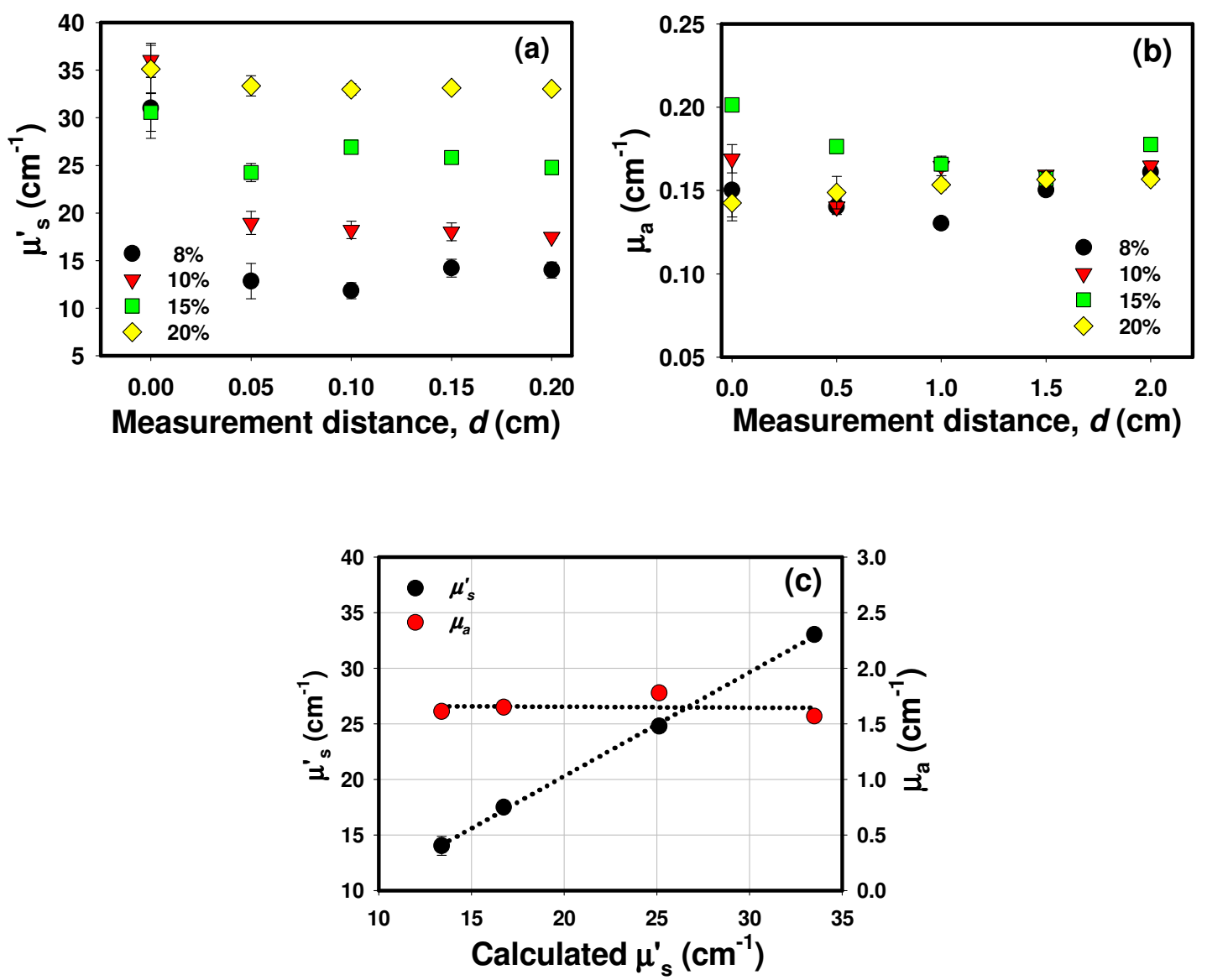

Figure 6-3: The determined (a) reduced scattering and (b) absorption coefficients versus the calculated reduced scattering coefficient $\mu_{s}$, for different intralipid solutions $20 \%, 15 \%, 10 \%$, and $8 \%$ at different measurement distances. (c) the determined reduced scattering and absorption coefficients for solutions with different intralipid concentrations at specific distance, $d=0.15 \mathrm{~cm}$. The detection angle $\phi$ is $60^{\circ}$. The dotted lines indicate the linear regression for the data. Error bars represent the $95 \%$ c.i. of the fitted values.

Figure 6-4(a-b) shows the reduced scattering and absorption coefficients for different India ink concentrations solutions at different measurement distances. The derived reduced scattering coefficients are not affected by the varying the India ink. The determined absorption coefficients is not consistent at small distance measurements $(\mathrm{d}<0.05 \mathrm{~cm})$; however, as the distance increases, the derived $\mu_{a}$ becomes stabilized. Figure 6-4(c) shows the reduced scattering and absorption coefficient for different intralipid concentration solutions at measurement distance $d=0.10 \mathrm{~cm}$. The India ink has no 
significant effect on the reduced scattering coefficient. For the absorption coefficient, increasing the India ink results in an increase in the derived absorption coefficient, where the slope is $0.944\left(\mathrm{R}^{2}=0.95\right)$.
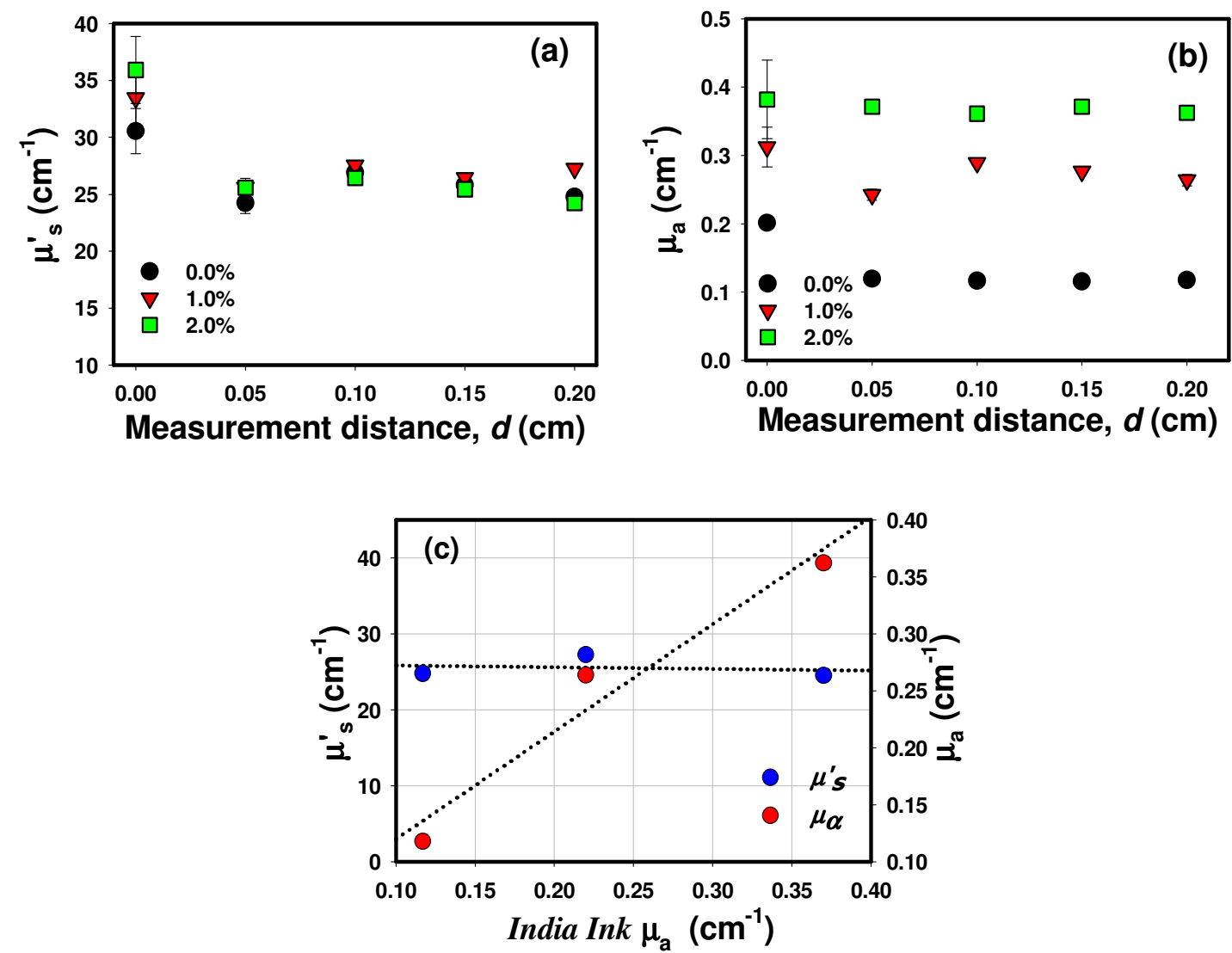

Figure 6-4: The determined (a) reduced scattering $\mu_{s}$, and (b) absorption $\mu_{a}$ coefficient versus the theoretical absorption coefficient for $15 \%$ intralipid solutions with different India ink $0.0 \%$, $1.0 \%$, and $2.0 \%$ (c) the determined reduced scattering and absorption coefficient for different intralipid solution at $d=0.2 \mathrm{~cm}$. The detection angle $\phi$ is $60^{\circ}$. The dotted lines indicate the linear regression for the data. Error bars represent the $95 \%$ c.i. of the fitted values.

The previous results demonstrated the validity of the device to determine the reduced scattering coefficient $\mu_{s}^{\prime}$ and absorption coefficient $\mu_{a}$. The $\mu^{\prime}{ }_{s}$ scale linearly with the intralipid concentration and the all the reduced scattering coefficient values are close to the theoretical values at large distances. The absorption scaled linearly with the India 
ink concentration. To be noted, the absorption coefficients for the India ink values are a bit higher than interoperated from the previous studies.

Figure 6-5(a) shows the optical path-length resolved reflectance of chicken liver obtained at different measurement distances. The reflectance curves trends are similar to the intralipid solution. Figure 6-5(b) shows the derived reduced scattering and absorption coefficient at different measurement distances. The reduced scattering and absorption coefficients become stable at large distances and their values at measurement distance $d=$ $0.2 \mathrm{~cm}$ are 9.72 and $1.13 \mathrm{~cm}^{-1}$, respectively.
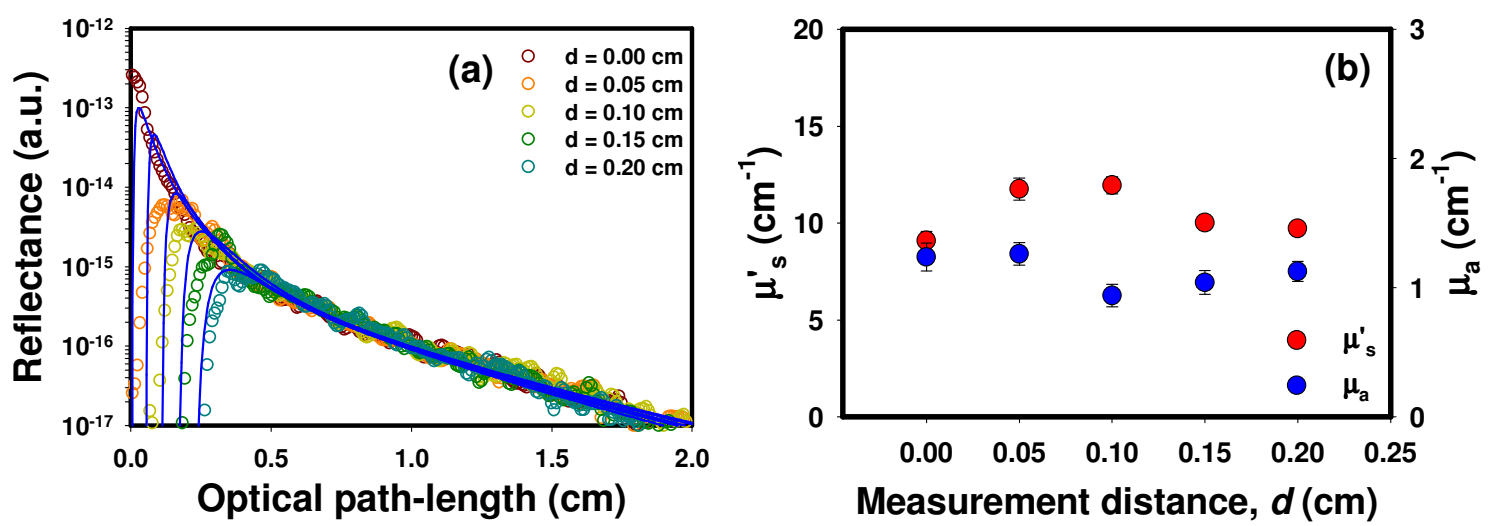

Figure 6-5: (a) The raw optical path-length resolved reflectance profile of chicken liver. (b) The retrieved reduced scattering $\mu_{s}^{\prime}$ and absorption $\mu_{a}$ coefficient at different measurement distances, $d=0.0,0.05,0.10,0.15$, and $0.20 \mathrm{~cm}$. Error bars represent the $95 \%$ c.i. of the fitted values.

Figure 6-6(a) and (b) show example of the path-length resolved reflectance profiles measured in tendon and their corresponding diffuse fit at different positions. The overall tendon profile is similar in trend as in Intralipid solution. At measurement angles $0^{\circ}, 22.5^{\circ}, 45^{\circ}$ and $67.5^{\circ}$, signals with sufficient signal-to-noise were obtained at 0.0 to 0.2 $\mathrm{cm}$ away from the incident point. When measuring perpendicularly to the fibers $(\alpha=$ $90.0^{\circ}$ ), the reflectance was detectable up to $d=0.40 \mathrm{~cm}$. Similar to the results in Intralipid solution (Fig. 6-2), the reflectance peak shifted to longer path-lengths and 
decreased in amplitude at larger measurement distances $(d)$.
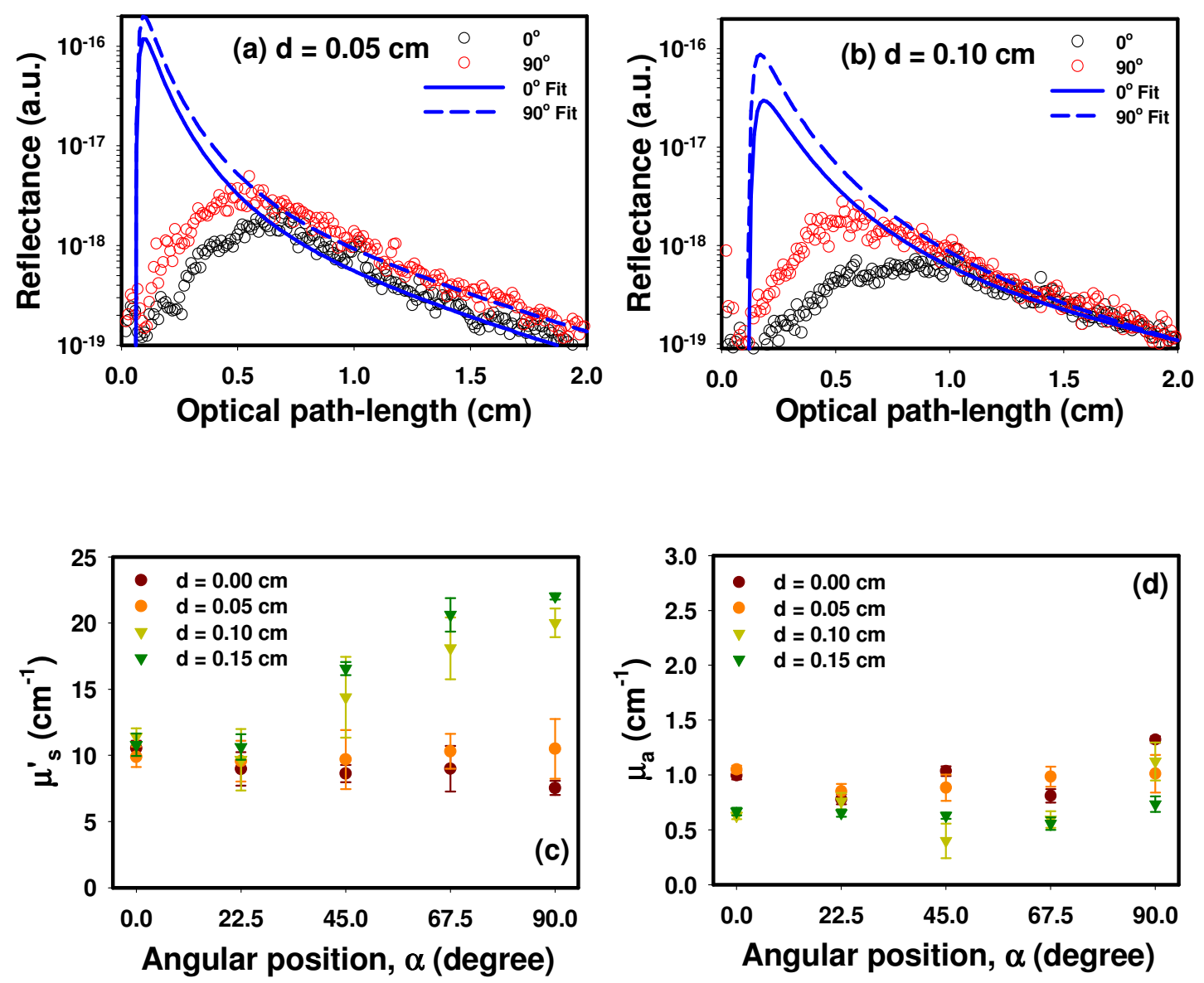

Figure 6-6: Path-length resolved reflectance measured in tendon and the corresponding diffuse fitting at measurement distance $d$ of (a) $0.05 \mathrm{~cm}$ and (b) $0.10 \mathrm{~cm}$, respectively. The retrieved (c) reduced scattering $\mu_{s}^{\prime}\left(\mathrm{cm}^{-1}\right)$ and (d) absorption $\mu_{a}\left(\mathrm{~cm}^{-1}\right)$ coefficients at different distances $d=$ $0.00,0.05,0.10$ and $0.15 \mathrm{~cm}$ and angular position $\alpha 0^{\circ}, 22.5^{\circ}, 45^{\circ}$, and $67.5^{\circ}$, and $90^{\circ}$. Error bars represent the $95 \%$ c.i. of the fitted values.

The corresponding diffuse fitting curves using Eq. (6-1) are also shown in Fig. 66(a) and (b). At $d=0.05 \mathrm{~cm}$, the fitting curves are almost proportional for parallel $\left(\alpha=0^{\circ}\right)$ and perpendicular $\left(\alpha=90^{\circ}\right)$ measurements; whereas at $d=0.10 \mathrm{~cm}$, the fitting curves differ significantly. It can be seen that when $d=0.10 \mathrm{~cm}$, the reflectance decayed faster than when measured perpendicularly to the fiber. 
Figure 6-6(c) shows the retrieved reduced scattering coefficient in tendon at different measurement positions. When the measurement location was close to the origin $(d=0.00 \mathrm{~cm})$, the reduced scattering coefficient was independent of the measurement orientation, which is similar to an isotropic media. The average reduced scattering coefficient when $d=0.00 \mathrm{~cm}$ was $\mu_{s}^{\prime}=10.7 \mathrm{~cm}^{-1}$. At larger measurement distances, the reduced scattering coefficient became strongly angular dependent. When $d=0.10 \mathrm{~cm}, \mu_{s}^{\prime}$ was the smallest $\left(\mu_{s}^{\prime} \approx 8.9 \mathrm{~cm}^{-1}\right)$ when measured along $\left(\alpha=0.0^{\circ}\right)$ the fiber direction; and reached the highest value $\left(\mu_{s}^{\prime} \approx 16.56 \mathrm{~cm}^{-1}\right)$ when measured perpendicularly $\left(\alpha=90^{\circ}\right)$ to the fiber direction. It can be seen that the confidence interval is quite large, which is due to the large noise in the measurement, especially at the small distances.

Figure 6-6(d) shows the retrieved absorption coefficient in tendon at different measurement positions. The determined absorption coefficient $\left(\mu_{a}\right)$ at all the different measurement distances appeared to be independent of the measurement direction, although the fitted values varied at different distance and angles. When $d=0.0 \mathrm{~cm}$, the average absorption coefficient was $\mu_{a}=0.99 \pm 0.22 \mathrm{~cm}^{-1}$; while it was $\mu_{a}=0.70 \pm 0.27$ $\mathrm{cm}^{-1}$ at $d=0.10 \mathrm{~cm}$.

\subsection{Discussion}

The isotropic diffuse equation fit the Intralipid and chicken liver results very well as shown in Figure 6-3(a) and Figure 6-5(a) at larger measurement distances. At smaller distances or smaller path-lengths, the fitting results were significantly larger than the theoretical values. Such discrepancy can be attributed to the fact that most detected 
photons with smaller path-lengths were not multiply scattered and thus didn't satisfy the diffuse approximation. This issue was more significant in the tendon tissue as shown in Figure 6-6, where the isotropic equation cannot fit the leading portion (rising) of the pathlength resolved reflectance despite the good fitting at longer path-lengths.

In tendon tissue, the obtained absorption coefficients did not show strong angular dependency, indicating insignificant anisotropic distribution in tissue absorption properties. Similarly, the fitted reduced scattering coefficients at small distances appeared to be isotropic. Therefore, these measurements likely were not affected by the anisotropic fibrous structures and may be indicators of background isotropic scatterings. However, the obtained $\mu_{s}^{\prime}$ showed strong variations with measurement angle at larger $(d>0.05 \mathrm{~cm})$ distances. In tendon tissue, the $\mu_{s}^{\prime}$ was smaller at $0^{\circ}$ and higher at $90^{\circ}$. This seems to be in agreement with general understanding of fibrous tissues because a cylindrical structure has minimal scattering along the cylinder axis. When measured along the fibers $\left(0^{\circ}\right)$, the obtained $\mu_{s}^{\prime}$ should be mainly from background isotropic scatters; whereas the $\mu_{s}^{\prime}$ obtained at $90^{\circ}$ contains contributions from both background and fibrous scatters (Shuaib and Yao 2010).

\subsection{Conclusion}

In summary, a Mach-Zehnder based low coherent interferometer was used to retrieve the optical properties of the fibrous structure (tendon). The raw path-length resolved reflectance showed strong angular dependency in tendon tissues. This study found that the derived absorption coefficient does not depend on the measurement 
direction. In contrast, the derived reduced scattering coefficient depends strongly on the measurement direction. Experimental observations in tendon sample support the existing knowledge on light scattering in fibrous materials. 


\section{CHAPTER 7}

\section{SUMMARY AND FUTURE DIRECTIONS}

This dissertation presents a study of optical reflectance in biological tissues that contain fibers, such as tendon, skin, dentin, and skeletal muscle. Such fibrous tissues were modeled using a two-scatter model: infinitely long cylinders representing fibers and spherical particles representing background cells, cell organelles, etc. A Monte Carlo model was applied to simulate the light propagation in such fibrous tissues. Both spatialand time-resolved measurements were obtained from the Monte Carlo simulation. A comparison between anisotropic diffuse theory and Monte Carlo simulation indicates that diffuse theory can correctly predict surface reflectance in fibrous tissues of small fibers. For larger fiber size, a correction factor needs to be used.

Both isotropic and anisotropic diffuse equations under different boundary conditions were compared in retrieving the optical properties of the cylindrical structure and the background in fibrous tissues. To apply isotropic diffuse theory, measurements both parallel and perpendicular to the fiber direction are needed; whereas, only one measurement perpendicular to fiber direction was needed for the anisotropic diffuse theory. The results indicate that isotropic diffuse equation under extrapolated-boundary condition had the best performance.

In experimental studies, a single-mode fiber optic Mach-Zehnder based low coherent interferometer (LCI) was implemented to measure time-resolved reflectance in scattering media. The results confirmed significantly different light propagation 
behaviors in isotropic and anisotropic media. Anisotropic diffuse theory can explain our observations in tendon, but not in skeletal muscle due to the additional structural variations in muscle. The isotropic diffuse theory was applied to retrieve the optical properties in tendon. The results showed that the reduced scattering coefficient is strongly dependent on the fiber direction; whereas absorption is independent of the fiber direction.

The simulation studies can be further improved. To simply the simulation, we assumed that fibers are perfectly aligned and have one size. However, in the biological tissue, both the fiber alignment and fiber size have some variations. These issues can be addressed by using a distribution to simulate inhomogeneous fiber orientation and size in the model. In addition, Monte Carlo simulation assumes independent scattering which may not be accurate in dense packed real tissues.

A significant difference was observed between experimental and simulation results. In anisotropic fibrous tissues, the leading portion of the experimental timeresolved reflectance is quite different from the simulation. The simulated results had a much steeper increase than the experimental results. This difference is more pronounced when the measurement position is far away from the incident light. Further investigation is needed to explain this difference.

Moreover, polarization was not considered in our current study. Polarization properties such as retardation and diattenuation strongly depend on anisotropic tissue structures. The Monte Carlo model can be modified to simulate the behaviors of polarized photons as they propagate through the medium. Polarization-sensitive 
measurements may provide additional information regarding the fiber size and orientation in fibrous tissues. 


\section{REFERENCES}

Backman, V., R. Gurjar, K. Badizadegan, I. Itzkan, R. R. Dasari, L. T. Perelman, and M. S. Feld, "Polarized light scattering spectroscopy for quantitative measurement of epithelial cellular structures in situ," Selected Topics in Quantum Electronics, IEEE Journal 5, 1019-1026 (1999).

Bannemann, C. G. and N. G. Laing, "Myopathies resulting from mutations in sarcomeric proteins," Current Opinion in Neurology 17, 529-537 (2004).

Bohern, C. F. and D. R. Huffman, Absorption and Scattering of Light by Small Particles (Wiley, New York, 1983), p. 544.

Case, K. M. and P. F. Zweifel, Linear transport theory (Addison-Wesley, Boston, 1967), p. 342.

Chan, W., P. Lang, M. Stevens, K. Sack, S. Majumdar, D. Stoller, C. Basch, and H. Genant, "Osteoarthritis of the knee: comparison of radiography, CT, and MR imaging to assess extent and severity," Am. J. Roentgenol. 157, 799-806 (1991).

Cheong, W. F., S. A. Prahl, and A. J. Welch, "A Review of the Optical-Properties of Biological Tissues.," IEEE J. Quantum Elect. 26, 2166-2185 (1990).

Choma, M. A., C. Yang, and J. A. Izatt, "Instantaneous quadrature low-coherence interferometry with $3 \times 3$ fiber-optic couplers," Opt. Lett. 28, 2162-2164 (2003).

Cubeddu, R., M. Musolino, A. Pifferi, P. Taroni, and G. Valentini, "Time-resolved reflectance: a systematic study for application to the optical characterization of tissues," Quantum Electronics, IEEE Journal 30, 2421-2430 (1994).

Dagdug, L., G. H. Weiss, and A. H. Gandjbakhche, "Effects of anisotropic optical properties on photon migration in structured tissues," Phys. Med. Biol. 48, 1361 (2003).

Delpy, D. T., M. Cope, P. V. D. Zee, S. Arridge, S. Wray, and J. Wyatt, "Estimation of optical pathlength through tissue from direct time of flight measurement," Phys. Med. Biol. 33, 1433-1442 (1988).

Dudko, O. K., G. H. Weiss, V. Chernomordik, and A. H. Gandjbakhche, "Photon migration in turbid media with anisotropic optical properties," Phys. Med. Biol. 49, 3979 (2004).

Eckstein, F. and C. Glaser, "Measuring Cartilage Morphology with Quantitative Magnetic Resonance Imaging," Semin Musculoskelet Radiol. 08, 329-353 (2004).

Fan, C., A. Shuaib, and G. Yao, "Path-length resolved reflectance in tendon and muscle," Opt. Express 19, 8879-8887 (2011). 
Farrell, T. J., M. S. Patterson, and B. C. Wilson, "A diffusion theory model of spatially resolved, steady-state diffuse reflectance for the non-invasive determination of tissue optical properties in vivo," Phys. Med. Biol. 19, 879-888 (1992).

Flock, S. T., S. L. Jacques, B. C. Wilson, W. M. Star, and M. J. C. V. Gemert, "Optical Properties of Intralipid: A phantom medium for light propagation studies " Lasers Surg. Med. 12, 510-519 (1992).

Forster, F. K., A. Kienle, and R. Hibst, "Determination of the optical parameters of dentin from spatially resolved reflectance and transmittance measurements," Proc. SPIE 4432, 103-109 (2001).

Frank, C., D. Bray, A. Rademaker, C. Chursch, P. Sabiston, D. Bodie, and R. Rangayyan, "Electron microscopic quantification of collagen fibril diameters in the rabbit medial collateral ligament: a baseline for comparison," Connect. Tissue Res. 19, 11-25 (1989).

Haskell, R. C., L. O. Svaasand, T.-T. Tsay, T.-C. Feng, M. S. McAdams, and B. J. Tromberg, "Boundary conditions for the diffusion equation in radiative transfer," J. Opt. Soc. Am. A 11, 2727-2741 (1994).

Hebden, J. C., J. J. G. Guerrero, V. Chernomordik, and A. H. Gandjbakhche, "Experimental evaluation of an anisotropic scattering model of a slab geometry," Opt. Lett. 29, 2518-2520 (2004).

Heino, J., S. Arridge, J. Sikora, and E. Somersalo, "Anisotropic effects in highly scattering media," Phys. Rev. E 68, 031908 (2003).

Hielscher, A. H., S. L. Jacques, L. Wang, and F. K. Tittel, "The influence of boundary conditions on the accuracy of diffusion theory in time-resolved reflectance spectroscopy of biological tissues," Phys. Med. Biol. 40, 1957 (1995).

Hielscher, A. H., H. Liu, B. Chance, F. K. Tittle, S. L. Jacques, "Time-resolved photon emission from layered turbid media,” Appl. Opt. 35, 719-728 (1996).

Ishimaru, A., Wave propagation and scattering in random media. (Academic Press, New York, 1978), p.600.

Jazrawi, L. M., M. J. Alaia, G. Chang, E. F. FitzGerald, and M. P. Recht, "Advances in Magnetic Resonance Imaging of Articular Cartilage," JAAOS 19, 420-429 (2011).

Jeffery, A., G. Blunn, C. Archer, and G. Bentley, "Three-dimensional collagen architecture in bovine articular cartilage," J. Bone Joint. Surg. Br. 73-B, 795-801 (1991).

Johnson, P. M., B. P. J. Bret, J. G. Rivas, J. J. Kelly, and A. Lagendijk, "Anisotropic Diffusion of Light in a Strongly Scattering Material," Phys. Rev. Lett. 89, 243901 (2002). 
Kadunce, D. P., R. Burr, R. Gress, R. Kanner, J. L. Lyon, and J. J. Zone, "Cigarette smoking: Risk factor for premature facial wrinkling," Ann. Intern. Med. 114, 840-844 (1991).

Kao, M. H., K. A. Jester, A. G. Yodh, and P. J. Collings, "Observation of Light Diffusion and Correlation Transport in Nematic Liquid Crystals," Phys. Rev. Lett. 77, 2233-2236 (1996).

Kastelic, J., A. Galeski, and E. Baer, "The Multicomposite Structure of Tendon," Connect. Tissue Res. 6, 11-23 (1978).

Keijzer, M., W. M. Star, and P. R. M. Storchi, "Optical diffusion in layered media," Appl. Opt. 27, 1820-1824 (1988).

Khan, K. M., J. L. Cook, N. Maffulli, and P. Kannus, "Where is the pain coming from in tendinopathy? It may be biochemical, not only structural, in origin," Br. J. Sports Med. 34, 81-83 (2000).

Kienle, A. and M. S. Patterson, "Improved solutions of the steady-state and the timeresolved diffusion equations for reflectance from a semi-infinite turbid medium," J. Opt. Soc. Am. A Opt. Image Sci. Vis. 14, 246-254 (1997).

Kienle, A., T. Glanzmann, G. Wagnieres, and H. Bergh, "Investigation of two-layered turbid media with time-resolved reflectance," Appl Opt 37, 6852-6862 (1998).

Kienle, A., F. K. Forster, R. Diebolder, and R. Hibst, "Light propagation in dentin: influence of microstructure on anisotropy," Phys. Med. Biol. 48, N7-14 (2003).

Kienle, A., F. K. Forster, and R. Hibst, "Anisotropy of light propagation in biological tissue," Opt. Lett. 29, 2617-2619 (2004).

Kienle, A., "Anisotropic light diffusion: an oxymoron?," Phys. Rev. Lett. 98, 218104 (2007).

Kienle, A., C. Wetzel, A. Bassi, D. Comelli, P. Taroni, and A. Pifferi, "Determination of the optical properties of anisotropic biological media using an isotropic diffusion model," J. Biomed. Opt. 12, 014026 (2007).

Kienle, A., C. D'Andrea, F. Foschum, P. Taroni, and A. Pifferi, "Light propagation in dry and wet softwood," Opt. Express 16, 9895-9906 (2008).

Kou, L., D. Labrie, and P. Chylek, "Refractive indices of water and ice in the 0.65 um 2.5um spectral range," App. Opt. 32, 3531-3540 (1993).

Laing, N. G. and K. J. Nowak, "When contractile proteins go bad: the sarcomere and skeletal muscle disease," Bioessays 27, 809-822 (2005). 
Lake, S. P., K. S. Miller, D. M. Elliott, and L. J. Soslowsky, "Effect of fiber distribution and realignment on the nonlinear and inhomogeneous mechanical properties of human supraspinatus tendon under longitudinal tensile loading," J. Orthop. Res. 27, 1596-1602 (2009).

Li, X., J. C. Ranasinghesagara, and G. Yao, "Polarization-sensitive reflectance imaging in skeletal muscle," Opt. Express 16, $9927-9935$ (2008).

Lieber, R., Skeletal Muscle Structure, Function \& Plasticity: The Physiological Basis of Rehabilitation, 2nd ed. (Lippincott Williams \& Wilkins, Philadelphia, 2002), p. 367

Lin, S.-P., L. Wang, S. L. Jacques, and F. K. Tittel, "Measurement of tissue optical properties by the use of oblique-incidence optical fiber reflectometry," Appl. Opt. 36, 136-143 (1997).

Mankin, H. and A. Thrasher, "Water content and binding in normal and osteoarthritic human cartilage," J. Bone Joint Surg. Am. 57, 76-80 (1975).

Marieb, E. and K. Hoehn, Anatomy and Physiology, 7rd ed. (Benjamin Cummings, San Francisco, CA, 2006), p. 1159.

Marquez, G., L. V. Wang, S.-P. Lin, J. A. Schwartz, and S. L. Thomsen, "Anisotropy in the absorption and scattering spectra of chicken breast tissue," App. Opt. 37, 798-804 (1998).

Matthew, C. and M. Moore, "Regeneration of rat extensor digitorum longus tendon: the effect of a sequential partial tenotomy on collagen fibril formation," Matrix 11, 259-268 (1991).

Michna, H., "Morphometric analysis of loading-induced changes in collagen fibril populations in young tendons.," Cell Tissue Res. 236, 465-470. (1984).

Minet, O., G. Muller, and J. Beuthan, Selected Papers on Optical tomography, Fundamentals and Applications in Medicine (SPIE press, 1998), Vol. MS 147.

Morgan, M., O. Kostyuk, R. A. Brown, and V. Mudera, "In Situ Monitoring of Tendon Structural Changes by Elastic Scattering Spectroscopy: Correlation with Changes in Collagen Fibril Diameter and Crimp," Tissue Eng. 12, 1821-1831 (2006).

Moseley, M., Y. Cohen, J. Kucharczyk, J. Mintorovitch, H. Asgari, M. Wendland, J. Tsuruda, and D. Norman, "Diffusion-weighted MR imaging of anisotropic water diffusion in cat central nervous system," Radiol. 176, 439-445 (1990).

Mourant, J. R., T. M. Powers, T. J. Bocklage, H. M. Greene, M. H. Dorin, A. G. Waxman, M. M. Zsemlye, and H. O. Smith, "In vivo light scattering for the detection of cancerous and precancerous lesions of the cervix," Appl. Opt. 48, D26-D35 (2009). 
Movin, T., A. Gad, F. Reinholt, and C. Rolf, "Tendon pathology in long-standing achillodynia: biopsy findings in 40 patients," Acta. Orthop. Scan. 68, 170-175 (1997).

Muller, G., B. Chance, R. Alfano, S. Arridge, J. Beuthan, E. Gratton, M. Kaschke, B. Masters, S. Svanberg, and P. van der Zee, "Medical Optical Tomography: Functional Imaging and Monitoring," in SPIE press, (Bellingham, WA, 1993).

Muller, G. and A. Roggan, eds., Laser-Induced Intersitial Thermotherapy (SPIE Press, Bellingham, WA, 1995), Vol. PM 25.

Newton, P., S. Woo, L. Kitabayashi, R. Lyon, D. Anderson, and W. Akeson, "Ultrastructural changes in knee ligaments following immobilisation.," Matrix 10, 314319 (1990).

Nickell, S., M. Hermann, M. Essenpreis, T. J. Farrell, U. Krämer, and M. S. Patterson, "Anisotropy of light propagation in human skin," Phys. Med. Biol. 45, 2873 (2000).

Nueman, A. P., "Articular cartilage repair," Am. J. Sports Med. 26, 309-324 (1998).

Parry, D. and A. Craig, Growth and development of collagen fibrils in connective tissue. In Ultrastructure of the Connective Tissue Matrix (Nijhoff, Boston, 1984), pp. 34-64.

Patterson, M. S., B. Chance, and B. C. Wilson, "Time resolved reflectance and transmittance for the non-invasive measurement of tissue optical properties," Appl. Opt. 28, 2331-2336 (1989).

Perelman, L. T., V. Backman, M. Wallace, G. Zonios, R. Manoharan, A. Nusrat, S. Shields, M. Seiler, C. Lima, T. Hamano, I. Itzkan, J. Van Dam, J. M. Crawford, and M. S. Feld, "Observation of Periodic Fine Structure in Reflectance from Biological Tissue: A New Technique for Measuring Nuclear Size Distribution," Phys. Rev. Lett. 80, 627 (1998).

Popescu, G. and A. Dogariu, "Optical path-length spectroscopy of wave propagation in random media," Opt. Lett. 24, 442-444 (1999).

Proske, U. and D. Morgan, "Muscle damage from eccentric exercise: mechanism, mechanical sign, adaptation and clinical applications.," J. Physio. 537, 333-345 (2001).

Ranasinghesagara, J., F. Hsieh, and G. Yao, "A photon migration method for characterizing fiber formation in meat analogs," J. Food Sci. 71, E227-231 (2006).

Ranasinghesagara, J. and G. Yao, "Imaging 2D optical diffuse reflectance in skeletal muscle," Opt. Express 15, 3998-4007 (2007).

Ranasinghesagara, J. and G. Yao, "Effects of inhomogeneous myofibril morphology on optical diffraction in single muscle fibers," J. Opt. Soc. Am. A 25, 3051-3058 (2008). 
Ranasinghesagara, J., T. M. Nath, S. J. Wells, A. D. Weaver, D. E. Gerrard, and G. Yao, "Imaging optical diffuse reflectance in beef muscles for tenderness prediction," Meat Sci. 84, 413-421 (2010).

Ross, M., G. Kaye, and W. Pawlin, Histology (Lippinocott Williams \& Wilkins, Baltimore, 2003), p. 875

Schafer, J. and A. Kienle, "Scattering of light by multiple dielectric cylinders: comparison of radiative transfer and Maxwell theory," Opt. Lett. 33, 2413-2415 (2008).

Schmitt, J. M. and G. Kumar, "Turbulent Nature of Refractive-Index Variations in Biological Tissue," Opt. Lett. 21, 1310-1312 (1996).

Shuaib, A. and G. Yao, "Equi-intensity distribution of optical reflectance in a fibrous turbid medium," App. Opt. 49, 838-844 (2010).

Shuaib, A. and G. Yao, "Determining the optical properties in a fibrous turbid medium," Proc. SPIE 7897, 78978Q (2011) doi:10.1117/12.873007.

Smith, J., E. Davidson, W. Sams, and R. Clark, "Alterations in human dermal connective tissue with age and chronic sun damage," J. Invest. Dermatol. 39, 347-350 (1962).

Stark, H. and T. C. Lubensky, "Multiple light scattering in nematic liquid crystals," Phys. Rev. Lett. 77, 2229 (1996).

Sviridov, A., V. Chernomordik, M. Hassan, A. Russo, A. Eidsath, P. Smith, and A. H. Gandjbakhche, "Intensity profiles of linearly polarized light backscattered from skin and tissue-like phantoms," J. Biomed. Opt. 10, 014012-014019 (2005).

Tuchin, V. V., Selected Papers on Tissue Optics: Applications in Medicial Diagnostics and Therapy (SPIE press, Bellingham, WA, 1994), Vol. MS 102.

Tuchin, V. V., Handbook of Optical Biomedical Diagnositcs (SPIE press, Bellingham, WA, 2002). Vol. PM 107

Tyler, J. A., P. J. Watson, H. L. Koh, N. J. Herrod, M. Robson, and L. D. Hall, "Detection and monitoring of prefressive degenrative of oseoarthritic cartilage by MRI," Acta. Orthop. Scan. 66, 130-138 (1995).

Varghese, B., V. Rajan, T. G. Van Leeuwen, and W. Steenbergen, "Path-length-resolved measurements of multiple scattered photons in static and dynamic turbid media using phase-modulated low-coherence interferometry," J. Biomed. Opt. 12, 024020-024027 (2007).

Venn, M. and A. Maroudas, "Chemical composition and swelling of normal and osteoarthritic femoral head cartilage," Ann. Rheum. Dis. 36, 121-129 (1977). 
Victoria, C. C., "Melanoma" (June, 30, 2009, 2010), retrieved Novermber, 10, 2010, http://www.cancervic.org.au/about-cancer/cancer_types/melanoma.

Vishwanath, K., H. Yuan, W. T. Barry, M. W. Dewhirst, and N. Ramanujam, "Using Optical Spectroscopy to Longitudinally Monitor Physiological Changes within Solid Tumors," Neoplasia 11, 889-900 (2009).

Vo-Dinh, T., Biomedical Photonics Handbook (CRC press, Boca Raton, 2003), p. 1872.

Wang, L. V., S. L. Jacques, and L. Zheng, "MCML--Monte Carlo modeling of light transport in multi-layered tissues," Comput. Methods Programs Biomed. 47, 131-146 (1995).

Wang, L. V. and H.-I. Wu, Biomedical Optics (Wiley-Interscience, Hoboken, NJ, 2007), p. 376.

Wang, X., B. W. Pogue, S. Jiang, X. Song, K. D. Paulsen, C. Kogel, S. P. Poplack, and W. A. Wells, "Approximation of Mie scattering parameters in near-infrared tomography of normal breast tissue in vivo," J. Biomed Opt. 10, 051704 (2005).

Watkins, S. C. and M. J. Cullen, "Muscle fiber size and shape in Duchenne muscular dystrophy," Neuropathol. Appl. Neurobiol. 8, 11-17 (1982).

Wax, A. and J. W. Pyhtila, "In situ nuclear morphology measurements using light scattering as biomarkers of neoplastic change in animal models of caricnogensis," Dis Makers 25, 291-301 (2008).

Williams, I., A. Craig, D. Parry, A. Goodship, J. Shah, and I. Silver, "Development of collagen fibril organization and collagen crimp patterns during tendon healing.," Int. J. Biol. Macromol. 7, 275-282. (1985).

Williams, W., Total Burn Care (Harcourt Publishers Ltd., San Diego, CA, 2002), Ch. 10, p. 776.

Xia, J., A. Weaver, D. E. Gerrard, and G. Yao, "Monitoring sarcomere structure changes in whole muscle using diffuse light reflectance," J. Biomed. Opt. 11, 040504 (2006).

Xia, J. and G. Yao, "Angular distribution of diffuse reflectance in biological tissue," Appl. Opt. 46, 6552-6560 (2007a).

Xia, J. and G. Yao, "Optical characterization of skeletal muscles," (University of Missouri-Columbia, Columbia, MO., 2007b).

Yamauchi, M., P. Prisayanh, Z. Haque, and D. T. Woodley, "Collagen cross-linking in sun-exposed and unexposed sites of aged human skin," J. Invest. Dermatol. 97, 938-941 (1991). 
Yousif, H. A. and E. Boutros, "A FORTRAN code for the scattering of EM plane waves by an infinitely long cylinder at oblique incidence," Comp. Phys. Comm. 69, 406-414 (1992). 


\section{VITA}

Ali Shuaib graduated from the Biological Engineering Department of the University of Missouri-Columbia in 2005. He received his Master in Science degree in 2007 in Biological Engineering from the University of Missouri-Columbia under the supervision of Dr. Mark A. Haidekker. Ali started his PhD under the supervision of Dr. Gang Yao in 2007 at the University of Missouri-Columbia. His research interest is tissue optics. 